\title{
Investigation of Shear Flow in a Planar Cylindrical Hybrid Geometry for Rheo-NMR Applications
}

by

Sarah Jane Stevenson

\author{
A thesis \\ submitted to the Victoria University of Wellington \\ in fulfilment of the \\ requirements for the degree of \\ Master of Science \\ in Physics.
}

Victoria University of Wellington

2018 



\begin{abstract}
Materials which exhibit peculiar behaviour due to applied mechanical deformations are abundant in everyday life. Rheo-NMR is an established technique which has been used to study these responses for the past three decades by combining methodologies from rheometry and nuclear magnetic resonance (NMR). The technique enhances standard rheological studies of bulk properties, such as viscosity and elasticity, by applying the tools of NMR (e.g. spectroscopy, diffusion, relaxometry, imaging, and velocimetry) to matter under deformation. This allows for the exploration of molecular origins and / or local responses within the material which lead to the macroscopic behaviour. These materials are deformed (most commonly sheared) inside geometric housings with a NMR experiment running in parallel. For complex material studies it is desirable for these geometries to provide a simple homogeneous deformation. In reality, all standard rheometry geometries have inhomogeneity characteristics. In fact there is evidence to suggest that some material responses may be influenced by a small degree of deviation from pure homogeneity. This makes it harder to isolate any inherent material behaviour due to a magnitude or rate of deformation from the specific characteristics of how the deformation was applied.

This contribution reports on the continued design and method development of a novel geometry for rheo-NMR - a planar cylindrical hybrid (PCH) shear geometry. The geometry includes planar sections with the aim to provide planar Couette flow, a simple truly homogeneous shear profile. It comprises of two parallel sections of planar flow connected by two semi-circular sections of circular flow to give a closed flow path in the shape of a racetrack. Shear is applied by rotating a band around the inner section like a conveyor belt.

The purpose of the $\mathrm{PCH}$ geometry is to study the complex responses of materials under shear in this atypical shear environment. A paragon of a model system for exploring the novel geometry is a shear banding wormlike micelle (WLM) solution. It has a well documented nonlinear response to steady shear and previous work demonstrated that the curvature of a standard concentric cylinder geometric housing influenced the observed WLM's rheological response. Strikingly, what was discovered by this thesis research was that there was no visible appearance of
\end{abstract}


this material separating into bands in the planar (or cylindrical) regions in the $\mathrm{PCH}$ geometry when probed with an NMR velocity encoded imaging experiment. The more Newtonian-like response of the complex material differs from the intriguing curved flow profile seen for an actual Newtonian sample (which additionally evolves over the planar region) meaning the WLM's response is still complex in nature. From these findings it is clear that geometry did not impart the homogeneous planar Couette flow for a Newtonian sample. However it has introduced a new deformation environment to study complex materials, acting completely differently to the geometries typically used in rheo-NMR and rheometry. Implications of this and motivation for work study are discussed. 


\section{Acknowledgments}

My Master's year of research was filled with many interesting discussions and exciting opportunities, which I was fortunate enough to experience. For this I am grateful, as it has helped me grow in confidence and made me even more curious to continue to learn, explore, and share.

Thanks must go to my two supervisors, Petrik Galvosas and Tim Brox. They pushed me towards challenging opportunities, but made sure that I had the right tools and support upon which to stand. I appreciate their time and patience whenever I would pop by with a 'quick question' that would inevitably turn into a discussion that was anything but. Petrik was always encouraging yet honest, helping me start to figure out my own way in the world of research, and he spent many hours kindly answering my questions when I was driven to know more and understand why (even when explaining the intricacies of imaging for the 8th time). Tim encouraged me to think creatively about my research, guided and challenged me to find my own answers, and inspired me with his handy knowledge and his articulate way of communicating it. I was never without full and detailed feedback on my work.

I must acknowledge both Finnian Gray and Tim Brox who kindly allowed me to present some of their results from the past work on the Planar Cylindrical Hybrid geometry, and a shear banding wormlike micelle, respectively.

From a construction stand point I am grateful for the precise and speedy work from Nick Grinter and the Victoria University of Wellington Mechanical Workshop, for tasks great and small. The sharing of experience for improving and realising the design key to this work, as well as hearing the explanation behind this thinking, was most helpful and interesting.

My research time was made all the more enjoyable by the friendly and welcoming environment created by the Magnetic Resonance Physics Group in which I conducted my research. Over my relatively short time with the group I have met people from almost every continent and have been entertained by wonderful stories and fascinating science chats. I look forward to future meetings. 
I was fortunate to go to three conferences during my masters; to the International Conference on Magnetic Resonance Microscopy (ICMRM) in Halifax, the Australian-Korean Rheology Conference (AKRC) in Sydney and the Australian and New Zealand Society for Magnetic Resonance Conference (ANZMAG) in Kingscliff. As such I would like to thank the wider NMR and Rheological community that I met at these events for the discussions, feedback, and general encouragement for a master's student. For those who kindly hosted me during these travels your generosity was as warm as your welcome.

I was also supported financially with my tuition covered by a Victoria Master's By Thesis Fees Scholarship from Victoria University of Wellington, and stipends provided by a Postgraduate Scholarship from Freemasons New Zealand and MBIE. I additionally received a financial prize and travel award from the ANZMAG conference.

A huge thank you must go to my parents Marina and Murray Stevenson for their continual support in all aspects of my life; with university, at home, and for everything else that I endeavour to fit into each day. The experiences I have had surrounding the international travel, in particular, would not have been possible without their support either in person or through our various phone calls and messages. Lastly I am most grateful to both my family and friends for encouraging me, for listening to my ups and downs, and for believing that I can exceed my own expectations. 


\section{Contents}

1 Introduction 1

2 Description of Rheo-NMR 5

2.1 Rheology . . . . . . . . . . . . . . . . . 5

2.1.1 Stress and Strain in Materials Under Shear . . . . . . . . . 6

2.1.2 Shear Geometries and their Impact on Deformations . . . . . 10

2.1.3 Complex Materials . . . . . . . . . . . . . . . . . . . . . 15

2.2 Nuclear Magnetic Resonance . . . . . . . . . . . . . . . . . . 17

2.2.1 Larmor frequency and Bloch-Torrey Equations . . . . . . . . 18

2.2.2 NMR Excitation and Detection . . . . . . . . . . . . . . . . 19

2.2 .3 Relaxometry . . . . . . . . . . . . . . . . . 20

2.2.4 Magnetic Field Gradients . . . . . . . . . . . . . . . . . 22

2.2 .5 The NMR Spectrometer . . . . . . . . . . . . . . . . . 26

2.3 Rheo-NMR . . . . . . . . . . . . . . . . . . . . . . . . . 28

2.3.1 Enhancing Rheological Studies with NMR . . . . . . . . . 28

2.3.2 Types of Hardware and Set Up . . . . . . . . . . . . . . . . 29

2.4 Case Study: Planar Shear Geometry . . . . . . . . . . . . . . . . . . . 31

2.4.1 Influence of Curvature in Taylor-Couette Geometry . . . . . . 31

2.4.2 Planar Cylindrical Hybrid Design . . . . . . . . . . . . 33

2.4 .3 Further Work on $\mathrm{PCH} \ldots \ldots$. . . . . . . . . . . 36

3 Experimental Methods $\quad 37$

3.1 Characterisation of Sample Fluids . . . . . . . . . . . . . . 37

3.1.1 Benchtop Rheometer . . . . . . . . . . . . . . . . . . . . . 37

3.1 .2 Doped Water . . . . . . . . . . . . . . . . . . . . . . 41

3.1.3 Wormlike Micelle Surfactant System . . . . . . . . . . . . . . 41

3.2 Data Acquisition and Processing . . . . . . . . . . . . . . . 45

3.2.1 Flow Volumes Sampled for 2D and 1D Plots . . . . . . . . . 45

3.2.2 Data Processing and Thresholding . . . . . . . . . . . 50 
3.2 .3 Pixel Location . . . . . . . . . . . . . . . . . . . . . . 51

3.3 NMR Velocimetry Considerations . . . . . . . . . . . . . . 52

3.3.1 Mechanical Wobble . . . . . . . . . . . . . . . . . . . . 52

3.3.2 NMR Repeatability . . . . . . . . . . . . . . . . . . . . . 53

3.3.3 Unwanted Magnetisation in a Single q-step . . . . . . . . . . 53

3.3.4 Image Flow Distortions . . . . . . . . . . . . . . . . 54

3.3.5 Optimising Timing Parameters . . . . . . . . . . . . . . . 54

4 Planar Cylindrical Hybrid (PCH) Geometry Development 57

4.1 Modifications to Previous Design . . . . . . . . . . . . . . . . . 57

4.1 .1 Plug - Alignment of Rotors . . . . . . . . . . . . . . . . . . . . . . . . . . 62

4.1 .2 Band Elastomer . . . . . . . . . . . . . . . . . . 62

4.1 .3 Interaction with RF coil $\ldots \ldots \ldots 63$

4.1.4 Improved PCH Device . . . . . . . . . . . . . . . . . . . 64

4.2 Temperature Control for NMR System . . . . . . . . . . . . . . . 67

$4.3 \mathrm{PCH}$ Preparation and Use . . . . . . . . . . . . . . . . 68

4.3 .1 Assembly . . . . . . . . . . . . . . . . . 69

4.3.2 Imaging Gradients Calibration . . . . . . . . . . . . . . 71

4.3 .3 Set Up Checks . . . . . . . . . . . . . . . . . . . 72

4.3 .4 Orientation and Flow $\ldots \ldots \ldots \ldots \ldots$

5 Shear Studies using PCH Geometry 77

5.1 Observed Planar Region Flow Patterns . . . . . . . . . . . . . . 77

5.1 .1 Evolution of Flow . . . . . . . . . . . . . . . . . . . 77

5.1 .2 Function of Shear Rate . . . . . . . . . . . . . . . . . . . . . . . . . . . 80

5.1.3 Comparison to Standard Geometries . . . . . . . . . . . . . . 82

5.2 Observed Cylindrical Region Flow . . . . . . . . . . . . . . . . 83

5.3 Additional Observations . . . . . . . . . . . . . . . . . . 85

5.3.1 Degrading WLM Sample . . . . . . . . . . . . . . . 85

5.3 .2 Variation in Gap . . . . . . . . . . . . . . . . 87

5.3 .3 Secondary Flow . . . . . . . . . . . . . . . . . . . . . . . . . . 88

5.3 .4 Backflow and Offset . . . . . . . . . . . . . . . 89

6 Future Work $\quad 91$

7 Conclusions $\quad 95$

7.1 Summary of Presented Work . . . . . . . . . . . . . . . 95

7.2 Final Remarks . . . . . . . . . . . . . . . . . . . . . . 96 
A Definitions

$\begin{array}{ll}\text { B Derivations } & 109\end{array}$

B.1 Continuity Equation . . . . . . . . . . . . . . . . . . 109

B.2 Momentum Balance Equation . . . . . . . . . . . . . . 110 


\section{List of Figures}

2.1 A Hookean solid box deformed by a shear stress acting on the top

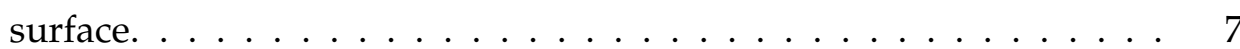

2.2 A Newtonian liquid sheared between two plates sliding relative to one another. . . . . . . . . . . . . . . . 7

2.3 Diagram of a sinusoidal oscillatory shear strain experiment showing the stress response separated into elastic and viscous components. . 9

2.4 TA Instruments AR 2000 stress controlled rheometer. . . . . . . . . 10

2.5 Homogeneous planar Couette flow between two infinite parallel plates. 11

2.6 Taylor-Couette geometry with an inner rotating and outer stationary wall. . . . . . . . . . . . . . . . . . . . . . 13

2.7 Examples of TC geometry velocity profiles over the sample gap with different inner and outer wall radii. . . . . . . . . . . . . . . . . 14

2.8 Cone and plate geometry. . . . . . . . . . . . . . . . . . . . 14

2.9 Flow curve shapes characteristic of shear thickening, shear thinning and a Newtonian fluid. . . . . . . . . . . . . . . . . . 16

2.10 Flow field and steady state flow curve characteristics for a shear banding material. . . . . . . . . . . . . . . . 17

2.11 PGSE-RARE pulse sequence used to record 2D velocity maps. . . . . 27

2.12 1D double slice selection pulse sequence used to record 1D velocity profiles. . . . . . . . . . . . . . . . . . 27

2.13 Bruker $400 \mathrm{MHz}$ NMR magnet. . . . . . . . . . . . . . . . . . . 28

2.14 Components of Rheo-NMR kit and how they are mounted in the magnet system. . . . . . . . . . . . . . . . . . 30

2.15 Description of original Planar Cylindrical Hybrid geometry design. . 34

2.16 Results (NMR and simulations) from preliminary testing of $\mathrm{PCH}$ with water at a shear rate of $4 /$ s. . . . . . . . . . . . . . 35

2.17 Velocity map showing a band distortion in planar regions for PDMS oil. . . . . . . . . . . . . . . . . . . . . . 36 
3.1 Temperature calibration test for AR 2000 rheometer. . . . . . . . . . . 39

3.2 Rheometer fill amounts testing using a shear banding sample. . . . . 39

3.3 Flow curve for the three batches of WLM solution. . . . . . . . . . . . 43

3.4 Frequency sweep data on the second batch of WLM solution at a $4 \%$ strain with a Maxwell model fit. . . . . . . . . . . . . . . . . . . . 43

3.5 Start up flow for WLM solution at $\dot{\gamma}=0.5 / \mathrm{s}, 5 / \mathrm{s}, 10 / \mathrm{s} . \ldots \ldots 4$

3.6 MRI dimension labels. . . . . . . . . . . . . . . . . . . . . . 45

3.7 Names for key imaging slice orientations for both the novel $\mathrm{PCH}$ and TC geometries. . . . . . . . . . . . . . . . . . . . 46

3.8 Examples of PGSE-RARE 2D velocity maps and images of TC and PCH geometries. . . . . . . . . . . . . . . . . . 47

3.9 Selection of planar region from 2D axial velocity map. . . . . . . . . . 48

$3.102 \mathrm{D}$ and corresponding averaged 1D cylindrical slice velocity maps for TC geometry. . . . . . . . . . . . . . . . . . . . . . 49

3.11 Spatially resolved propagator from $1 \mathrm{D}$ velocity data. . . . . . . . . . . 49

3.12 Example of 1D velocity data extracted over a single sample gap for a range of shear rates. . . . . . . . . . . . . . . . . . 51

3.13 Example of image flow distortions in TC geometry. . . . . . . . . . 55

4.1 Illustration of first iteration of $\mathrm{PCH}$ shear geometry with labelled components and nominal dimensions. . . . . . . . . . . . . 58

4.2 Misalignment in first iteration of $\mathrm{PCH} \ldots \ldots$. . . . . . . . . . . 60

4.3 Plug piece design. . . . . . . . . . . . . . . . . . . . 61

4.4 RF coil attachment ring design for $\mathrm{PCH}$. . . . . . . . . . . . . . 65

4.5 Improved $\mathrm{PCH}$ device (photo and main imaging slice orientation MRIs). . . . . . . . . . . . . . . . . . . . . 66

4.6 Coldstream Air Gun assembly at NMR magnet (not connected). . . . 68

4.7 A study on the effect of the number of turns of the gear cover screws on the fit between rotor ends and tapered holes. . . . . . . . . 70

$4.8 \mathrm{PCH}$ geometry with and without stabilising insert. . . . . . . . . . 71

4.9 Orientation and flow marker hole. . . . . . . . . . . . . . . . . 74

4.10 Example of experiments used to determine orientation and flow for imaging slices. . . . . . . . . . . . . . . 75

4.11 Orientation and flow of the three main imaging slices: axial, planar, and cylindrical. . . . . . . . . . . . . . . . 76 
5.1 Evolution of flow along planar region in PCH for WLM solution and doped water (2D axial velocity maps). Velocity profiles at ends and center of region are extracted.

5.2 2D displayed velocity distribution for planar slices of PCH geometry with water sheared at $\dot{\gamma}=5 / \mathrm{s}$ showing the evolution of the shape of the velocity profile along the planar region. . . . . . . . . . 80

5.3 Velocity profiles across the gap taken at the center of planar region in the $\mathrm{PCH}$ geometry for a range of shear rates with WLM and doped water. . . . . . . . . . . . . . .

5.4 Velocity profiles across the gap in the TC geometry for a range of shear rates with WLM and doped water. . . . . . . . . . 83

5.5 Flow in cylindrical regions of $\mathrm{PCH}$ geometry at a shear rate of $\dot{\gamma}=10 / \mathrm{s}$ with WLM and doped water. . . . . . . . . . . . . . . 84

5.6 Velocity profile across the cylindrical gap in the $\mathrm{PCH}$ geometry for a range of shear rates with WLM. . . . . . . . . . . . . . 85

5.7 Velocity profiles across the gap taken at the center of planar region in the PCH geometry for a range of shear rates with degraded WLM. 86

5.8 ParaVision MRI showing variation in gap thickness. . . . . . . . . . 87

5.9 Example backflow in the $\mathrm{PCH}$ geometry. . . . . . . . . . . . . . . 89 


\section{List of Tables}

3.1 Viscosity data for the three silicone oils measured by the TA Instruments rheometer compared to the stated nominal viscosities of the product. . . . . . . . . . . . . . . . 40

4.1 Set of preparation MRI experiments for $\mathrm{PCH}$ and what each one checks. . . . . . . . . . . . . . . . . 73

5.1 Imaging resolutions for the 1D velocity plots collapsed down from $2 \mathrm{D}$ velocity map data. . . . . . . . . . . . . . . . . . . 81 


\section{Chapter 1}

\section{Introduction}

When does a solid behave like a liquid, when does a liquid behave like a solid? Both of which are seemingly counter-intuitive questions on the surface but are vastly more complex and intriguing the deeper we look. Traditionally, in condensed matter physics, matter can be described as existing in a solid or liquid state. Solids behave elastically under an applied force, deforming a fixed amount, while liquids exhibit viscous behaviour and flow. While this categorisation of liquid or solid is applicable for describing simple molecular systems it fails for more complex materials which lie in the spectrum between these two states.

Understanding materials that display both elastic and viscous traits is of great interest as these materials are prominent in our everyday lives. The humble mayonnaise and custard, for instance, exhibit quite contrasting behaviours to applied forces: one becomes increasingly spreadable, the other forms surface that can be walked on. Outside the world of food science many cosmetic materials also display such complexity, so too for some biological fluids, asphalt, paints, gels, polymers, emulsions, and liquid crystal systems (e.g. LCDs), as well as fluids key to the fields of geophysics and engineering [see 1, Chapter 1.1]. Exploring their dynamic behaviour will not only help improve how these materials are processed and incorporated into products, but is additionally of interest to further understand condensed matter physics.

Flowing, stretching, ejecting, shearing, and otherwise deforming these materials is studied by rheology. Here the aim is to understand the dynamic behaviour by characterising it on a macroscopic scale, and relating this to microscopic features. Assisting to make this microscopic connection is an abundance of experimental techniques to partner with rheology: particle tracking velocimetry, dynamic light scattering, flow birefringence, ultrasonic speckle velocimetry, small angle neutron scattering, and nuclear magnetic resonance (NMR) [2]. 
Of these techniques rheo-NMR has a unique advantage due to its versatility. It lacks the need for tracker / scattering particles or optically / acoustically transparent samples. NMR uses the manipulation of nuclear spins to probe many soft matter properties that could be of interest for rheology. A focus here is on velocimetry which can produce velocity information mapped onto spin density images (magnetic resonances images or MRI's).

Geometric housings, which are used in experiments to deform samples, have some flow inhomogeneity characteristics coupled to their overall general practicability. These are magnified in rheo-NMR applications [3] and so this thesis aims to advance the field through the design and implementation of new hardware and experimental protocols. Specifically, this involves the continued development and application of a novel shear geometry, with a homogeneous flow in mind [see 4].

Relevant background material and techniques are introduced in chapters 2-3, original work makes up chapters $4-5$, and chapter 6 concludes. For completeness work presented in this thesis is from this master's project as well as the brief time preceding as a research assistant. The research assistant work concerned gaining some familiarity with NMR techniques and exploration of the original novel geometry device which led to ideas for the design development. A list of definitions for some key terms pertaining to this thesis can be found in Appendix A.

\section{Chapter 2: Description of Rheo-NMR}

In this chapter the field of rheo-NMR is introduced. Firstly through its component fields, rheology (the study of deformation and flow) and NMR (nuclear magnetic resonance), and then on how rheo-NMR brings those two topics together. In the rheology section relevant terminology, complex materials, and constitutive equations to describe material behaviour (in context of different types of matter and deformation geometries) are defined, and a rheological phenomena relating to this thesis work (shear banding) is introduced. NMR is described semi-classically, working up to relaxometry (spin relaxation measurements), imaging, and velocity encoding experiments. Early work in rheo-NMR and a brief discussion of its growth (along with standard hardware) is used to introduce the merger of the two fields. Finally special attention is given to a novel deformation geometry for rheoNMR that forms the basis for the research presented in this thesis.

\section{Chapter 3: Experimental Methods}

Experimental methods that are independent from the specific use of the novel shear geometry are described in this chapter. This includes an introduction to the testing 
materials, with a focus on their rheological and NMR properties, incorporating the rheology testing techniques. From the NMR analysis perspective, the types of experiments, how they can be processed, and the format that they will be displayed later in this work, are established. Wrapping up is a discussion on experimental considerations and potential sources of error.

\section{Chapter 4: Planar Cylindrical Hybrid (PCH) Geometry Development}

Chapter 4 is concerned with the development of the novel deformation geometry for rheo-NMR, the Planar Cylindrical Hybrid geometry, introduced in chapter 2. The aim is to improve the alignment and general ideality of the geometry. These improvements come with a required evolution of the methodology of use. As the system environment plays a role in the accuracy of experiments, a discussion is also had on temperature control, imaging calibration, and linking the physical geometry and its dynamics to processed NMR images.

\section{Chapter 5: Shear Studies using PCH Geometry}

The penultimate chapter in this thesis presents results of a complex shear banding material's behaviour using the developed design of the novel geometry and methodologies discussed in previous chapters. These are in the form of flow maps (2D images with velocity information) and velocity profiles (1D images with velocity information) from specific sections of the geometry, with a particular focus on the planar region. An attempt to characterise the flow environment was made with a simple fluid. Concluding is a section discussing additional observations from the shear studies.

\section{Chapter 6: Future Work}

This chapter includes a series of short discussions on possible future avenues of study based off the novel geometry design and implementation.

\section{Chapter 7: Conclusions}

To conclude the thesis, the work presented in the previous chapters is summarised. The closing remarks put the novel geometry's development in the context of the rheo-NMR studies done by the Magnetic Resonance Physics research group. 


\section{Chapter 2}

\section{Description of Rheo-NMR}

The work outlined in this thesis falls under the field of rheo-NMR, in particular rheo-NMR hardware development and applications. Rheo-NMR combines methodologies from rheology / rheometry and nuclear magnetic resonance (NMR) so it is pertinent to give a brief introduction to these two respective fields first, a prelude to rheo-NMR. Their descriptions will be kept to a level appropriate to understand the discussions and experimentation included in this thesis. What concludes is a case study of previous work on a novel piece of rheo-NMR hardware that will be further developed and explored in later chapters.

\subsection{Rheology}

Rheology is a branch of mechanics that was officially recognized as the study of deformation and flow of matter $[5,6]$ in the late 1920s. The name was coined by E. C. Bingham earlier in that decade [7], stemming from the Greek verb for flow, ṕćw. While the term rheology could be interpreted as quite broad (including both fluid dynamics and elasticity theory), the definition is more refined, focusing instead on complex materials that lie between the realms of classical elastic solids (Hookean) and classical viscous liquids (Newtonian). In particular, rheology studies the properties of these materials which determine their response to mechanical stimuli. As discussed by Barnes et al. [8], this was motivated by the emergence of discoveries in the 19th century revealing that such 'in-between' viscoelastic materials existed. Viscoelastic matter, which exhibits both traditional solid and liquid traits, leading to non-linear mechanical behaviour, is not only of interest from a condensed matter physics research perspective, but from an industrial and commercial view point as well. 
Physically, rheometry (pertaining to the measurement of rheological properties) studies bulk material responses, like viscosity and elasticity. Mechanical deformations to explore this can be applied in a variety of ways; from extensions to shear, and more specifically in the shape and texture of the sample environment. In contrast to fluid dynamics which uses simple fluids and complex flows, rheology explores complex materials in simple flows in an attempt to isolate the inherent material behaviour [7] to assist with analysis.

In this section basic rheological terms and expressions will be introduced, a discussion will be had on the two specific simple environments used in rheology to deform materials, and finally the idea of a complex material will be expanded upon, with a focus on the phenomena that materials used in this research express. Rheology is a diverse field in that it brings together a miscellany of sciences (from geophysics to biology to mathematics and beyond) so the curious reader is directed towards more comprehensive introductions to rheology which can be found in various rheology textbooks [7-12].

\subsubsection{Stress and Strain in Materials Under Shear}

As mentioned earlier, rheology is the study of deformation and flow. This can be quantified in terms of physical variables: strain (deformation), strain rate (flow), and stress.

To define these variables consider a volume of material trapped between two parallel surfaces, each with area $A$. A force, $F$, is applied to the top face acting along the plane of the surface. This results in a stress, $\sigma$, acting on the material defined as the force applied cross-section per unit area $\sigma=F / A$, with units of pressure $\mathrm{Pa}$. Specifically this is a shear stress as it acts parallel to the surface (a normal stress acts perpendicular). Stress can be thought of as a measure of the balance of the internal distribution of forces between neighbouring particles reacting to a load.

In the case of a solid it is possible to classify a strain (Figure 2.1) that results from the applied stress. A strain is a relative deformation, and is defined as the ratio of the distance the object deforms to the original length of the side of the object that is being deformed. These are $l$ and $L$ respectively, and are perpendicular in the shear case, giving a strain $\gamma=l / L$. The stress and strain are related by

$$
\sigma=G \gamma
$$

where the proportionality factor is the elastic or rigidity modulus $G$. This defines the behaviour of a classical Hookean solid. 


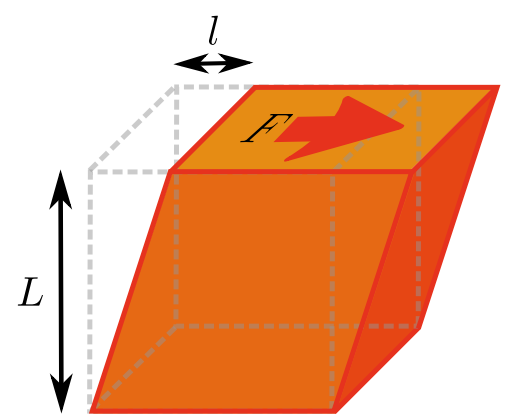

Figure 2.1: A Hookean solid box is deformed by a shear stress acting on the top surface. The height of the deformed side is $L$ and the distance deformed is $l$.

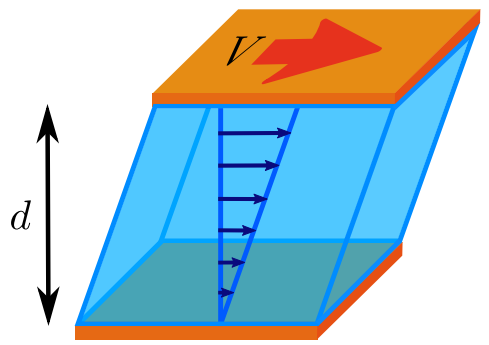

Figure 2.2: A Newtonian liquid is being sheared between two plates separated by a distance $d$ and sliding relative to one another with a force $F$ producing a velocity $V$. Arrows indicate the velocity gradient produced in the liquid. The plates each have surface area $A$.

Conversely in the case of a liquid, it is possible to classify a strain rate (Figure 2.2). A strain rate is a relative flow, and is defined to be the ratio of the change in the distance the object flows (velocity $V$ acting on the surface due to the moving plate) over the length that the velocity is distributed over (separation $d$ ). The strain rate is then $\dot{\gamma}=V / d$. It can be thought of as a description for the velocity profile across the sample gap (indicated by the blue arrows). In the shear case the strain rate is typically referred to as the shear rate. The stress and shear rate are related by

$$
\sigma=\eta \dot{\gamma}
$$

where the proportionality factor is the viscosity, $\eta$. This defines the behaviour of a classical Newtonian liquid. In particular, viscosity is a liquid's resistance to flow. 
For a simple Newtonian fluid this is constant (or a function of just temperature), however in more complex cases this can be a function of deformation parameters like time, shear rate, etc.

In 3D material cases stress is a tensor with components $\sigma_{i j}$

$$
\boldsymbol{\sigma}=\left(\begin{array}{lll}
\sigma_{11} & \sigma_{12} & \sigma_{13} \\
\sigma_{21} & \sigma_{22} & \sigma_{23} \\
\sigma_{31} & \sigma_{32} & \sigma_{33}
\end{array}\right)
$$

where the first indices denotes the surface plane being acted on (defined by its nor$\mathrm{mal}$ ), and the second, the direction of the force. The diagonal components are normal stresses and the off diagonal components are the shear stresses. ${ }^{1}$ Additionally, conservation of angular momentum means that $\sigma_{i j}=\sigma_{j i}$ [7]. In examples stated previously and explicitly in terms of equations (2.1) and (2.2), the stress is the $\sigma_{12}$ component of the stress tensor, an off diagonal component (signifying that they are both shear cases).

In studying material responses to applied mechanical stimuli it is the aim to develop material relations to characterise how the sample behaves. These are called constitutive equations which define the relationship between force and deformation, such as equations (2.1) and (2.2), for classical solid and liquid behaviour respectively. Matter must also obey conservation of momentum and mass which results in the equations of motion and the continuity equations derived in Appendices B.1 and B.2. For laminar incompressible flow these are

$$
\begin{aligned}
\frac{\partial}{\partial t} \rho \boldsymbol{v} & =\nabla \cdot \boldsymbol{\sigma}+\rho \boldsymbol{g} \\
\nabla \cdot \boldsymbol{v} & =0
\end{aligned}
$$

respectively, where $\rho$ is the density, $\boldsymbol{v}$ the velocity vector, and $\boldsymbol{g}$ the gravity vector.

Viscoelastic materials have their viscous and elastic responses acting on different time scales. Materials that may be thought of as solid (ball of silly putty or sheet of glass) will show instantaneous deformations on short timescales but will flow or creep on longer timescales. Hence a measure of viscoelasticity can be the relaxation modulus

$$
G(t)=\frac{\sigma(t)}{\gamma(t)}
$$

\footnotetext{
${ }^{1}$ The reader should be aware that some rheological texts [e.g. 7] split this into a sum of an extra or viscous stress tensor $\tau$ and a $-p \boldsymbol{I}$ tensor representing the hydrostatic pressure. This does not change the off diagonal components, and since normal stress differences (not explicit normal stress) are measured in rheological experiments this does not effect measured results of stress.
} 
Sinusoidal oscillatory shear deformation experiments measure this in terms of a stress response that is out-of-phase with the applied deformation. This makes the relaxation modulus a complex function, $G(t)=G^{\prime}+G^{\prime \prime}$, with strain and strain rate components, see Figure2.3. The component of the response that is in-phase with the deformation, $G^{\prime}$, is the storage modulus that represents the material elastically storing energy. Conversely the component of the stress completely out-of-phase with the deformation $G^{\prime \prime}$, is the loss modulus that represents the material viscously dissipating energy ${ }^{2}$.
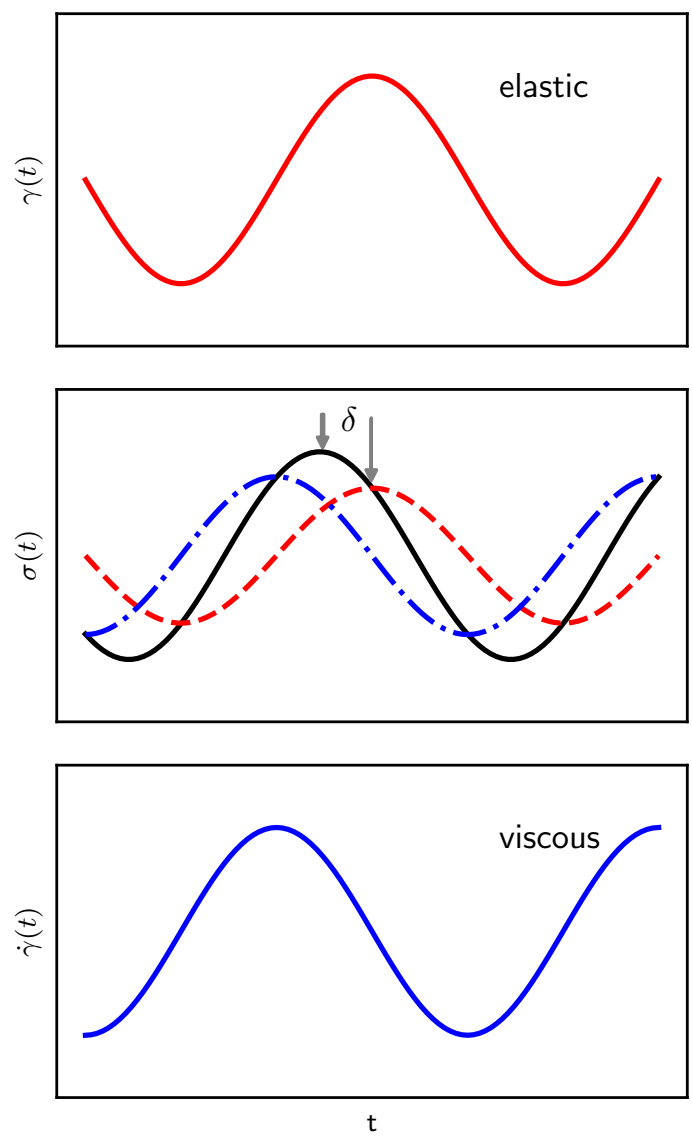

Figure 2.3: Diagram of a sinusoidal oscillatory shear strain experiment showing the stress response in the middle panel. For analysis the stress wave is separated into components in-phase with the strain (elastic - red) and strain rate (viscous - blue). The phase shift helps indicate how elastic or viscous the material is behaving.

\footnotetext{
${ }^{2}$ The viscoelastic behaviour described by $G^{\prime}$ and $G^{\prime \prime}$ can be related to a characteristic relaxation time discussed in Section 3.1.1 page 41.
} 


\subsubsection{Shear Geometries and their Impact on Deformations}

Rheometry is the physical measurement of rheological properties. The instrumentation used by rheologists is a rheometer which comprises a geometric configuration to house and deform the sample, and the mechanics to apply and measure stress, strain, strain rate, and normal forces (through force rebalance transducers, driver motor and air bearings etc.). This allows for measurements of viscosity, elastic moduli, and normal stress differences. Rheometers can be stress controlled or strain controlled (the rheometer applies a stress and measures a strain or the reverse). For this research a TA Instruments AR 2000 stress controlled rheometer shown in Figure 2.4 was used.

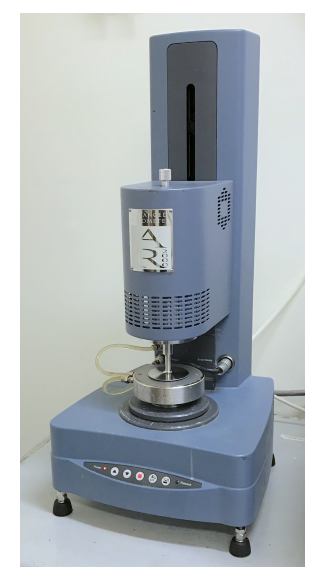

Figure 2.4: TA Instruments AR 2000 stress controlled rheometer used in this thesis. A $60 \mathrm{~mm}$ diameter $2^{\circ}$ cone and plate shear geometry was used for all testing.

Rheometers can have a large variety of geometries with which to apply the mechanical stimuli. Typically they are separated into two types, shear and extensional geometries. Within the range of shear geometries, there are drag flow (where the shear is generated by the relative motion between stationary and moving walls), and pressure driven flow (generated by a pressure difference along a channel) designs.

Drag flow geometries make use of sliding or rotating elements to apply a shear deformation to the sample trapped between two surfaces. These geometries include sliding plates, falling cylinder, falling ball, rotating ball, concentric cylinder (also referred to as a Taylor-Couette geometry), cone and plate, parallel disks, as well as drag flow indexers and eccentric rotating geometries. These are all introduced, along with equations describing their impact on materials in terms of instrument dimensions, and comments on when they are most applicable to material studies, in Macosko [7, Chapter 5]. 
With the exception of the last two on that list, the aim of many deformation geometries used in rheometry is to apply a simple shear. As stated earlier, this isolates material responses from the specifics of the deformation geometry, allowing for an easier analysis to establish constitutive relationships for the complex materials under study. To achieve the ideal shear case, the required geometry comprises two infinite parallel plates sliding relative to one another, like in Figure 2.5, which leads to a homogeneous sample deformation. This deformation defines planar Couette flow for a Newtonian fluid, named after French physicist Maurice Couette. Physically realising this experimental set up is problematic, even once the infinite property is removed, as discussed in Macosko [7, Section 5.2]. It is desirable to reduce some of these other problems concerning sample containment, end effects, and size impacting the range of measurements.

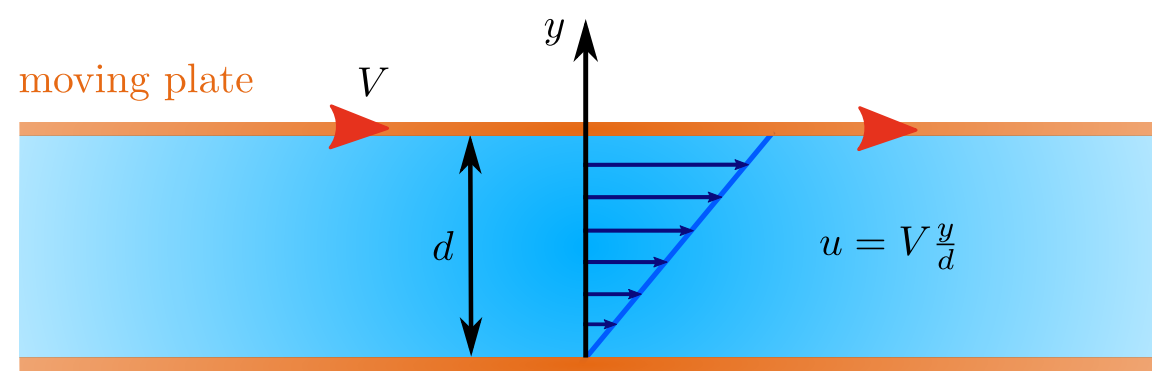

stationary plate

Figure 2.5: Homogeneous planar Couette flow between two infinite parallel plates. The planar Couette flow is shown as a linear velocity profile, $u$, between the stationary and moving wall.

In light of this, a common approach in the design of shear geometries is to use rotational symmetry to create closed flow lines to continuously shear the sample, while also approaching an approximate homogeneous flow field. Two such examples that are perhaps most commonly used are the concentric cylinder geometry and the cone and plate geometry. They are of particular interest in this work as the cone and plate geometry is used with the rheometer to characterise samples, and the concentric cylinder has been adapted for Rheo-NMR applications (see Section 2.3) which is then used as a comparison to the novel geometry developed in this thesis.

Note that the derivations and comments for these two geometries can be found in most Rheology books, see the recommend reading list above as well as Constaninescu [13]. 


\section{Concentric Cylinder (Taylor-Couette)}

For the concentric cylinder geometry the sample is trapped in the annulus of two concentric cylinders and the shear is applied by the relative rotating motion of the cylinders. As a rheological tool, the deformation can come from rotating (or letting rotate) either one of the cylinders while the stress, usually in the form of a torque can be measured (or applied) on the moving or stationary cylinder. The inner cylinder rotating is the Searle mode [14] while the outer cylinder rotating is the Couette mode [15, via [7]]. Modern rheometers may be able to do both i.e. counter rotation ${ }^{3}$. Taylor was the first to describe a mathematical representation of fluid instabilities in a concentric cylinder design with a moving inner wall which has led to the name Taylor-Couette flow to describe the flow generated between rotating cylinders. This is perhaps why a commonly used name for a modern iteration of the design is a Taylor-Couette geometry, in spite of the tendency for it having the inner wall rotating. In keeping with this trend it will be referred to as such or by the abbreviation TC from now on.

The arrangement of interest for acting as a benchmark geometry is one with a moving inner wall, a stationary outer wall, and strain controlled. For derivations it is chosen theoretically to take measurements of torque at the inner wall ${ }^{4}$. Considering a section of this geometry shown in Figure 2.6 we can label the dimensions in cylindrical coordinates, making use of the inherent symmetry of the design, where $r_{i}$ and $r_{o}$ are the radii of the inner and outer cylinder respectively, and $\Omega$ is the angular velocity of the rotating inner cylinder.

From equation (2.3) in cylindrical coordinates (see Appendix B.2) and laminar flow, with the $\theta$ component of velocity the only non-zero one, the inner wall rotating at $v_{\theta}\left(r_{i}\right)=r \Omega$, and negligible end effects, the shear stress is found to vary as a function of distance across the gap, yielding

$$
\frac{\sigma_{r \theta}\left(r_{i}\right)}{\sigma_{r \theta}\left(r_{o}\right)}=\frac{r_{o}^{2}}{r_{i}^{2}}
$$

as the ratio of shear stress between the outer and inner walls. A position dependence means that the shear will not be homogeneous. It can be approximated so if the radii are in the approaching limit of one another.

To get an absolute expression in terms of shear rate (this is what it applied to the sample during experiments) the assumption is made that the material in the

\footnotetext{
${ }^{3}$ See Anton Paar's MCR 702 TwinDrive rheometer.

${ }^{4}$ This is neither the original Couette or Searle design. Searle had the same rotating cylinder arrangement, however his device applied a stress to the material and the rotation rate of the inner cylinder was measured. The similarity to Couette's design is that we apply a strain and measure a stress response.
} 


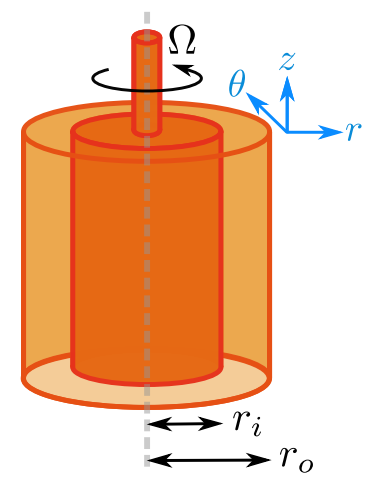

Figure 2.6: Concentric cylinder, or Taylor-Couette geometry, with an inner rotating and outer stationary wall to shear the sample.

geometry is an incompressible simple fluid and use the Navier-Stokes equations. This leads to a shear rate of

$$
\dot{\gamma}(r)=\frac{2 \Omega}{r^{2}} \frac{r_{i}^{2} r_{o}^{2}}{r_{o}^{2}-r_{i}^{2}}
$$

Even this simple fluid case does not give a constant shear rate. The narrow gap approximation is often made that $r_{o} \approx r_{i}$. This translates to the gap being sufficiently small, quantified by the dimensionless quantity $\kappa$ where

$$
\kappa=\frac{r_{i}}{r_{o}}
$$

representing the ratio of curvature or geometric factor. If $\kappa>0.99$ then the effects due to the curvature of the wall can be neglected. This leads to what is typically quoted as the constant applied shear rate

$$
\dot{\gamma}(r)=\frac{\Omega r_{i}}{r_{o}-r_{i}} .
$$

While $\kappa>0.99$ may be approached in some physical cases the stress or strain will still vary across the gap, meaning that this geometry will not have idealised homogeneous Couette flow. Later on there will be examples where this becomes a problem.

In terms of visualising the velocity profile without the narrow gap approximation the function is

$$
v_{\theta}(r)=\frac{\Omega r_{i}^{2}}{r_{o}^{2}-r_{i}^{2}}\left(\frac{r_{o}^{2}}{r}-r\right)
$$

where the non-linear component is visible.

Plotting this compared to the idealised linear velocity profile that corresponds to homogeneous flow from a steady shear rate shows the impact of the geometry dimensions in Figure 2.7. 


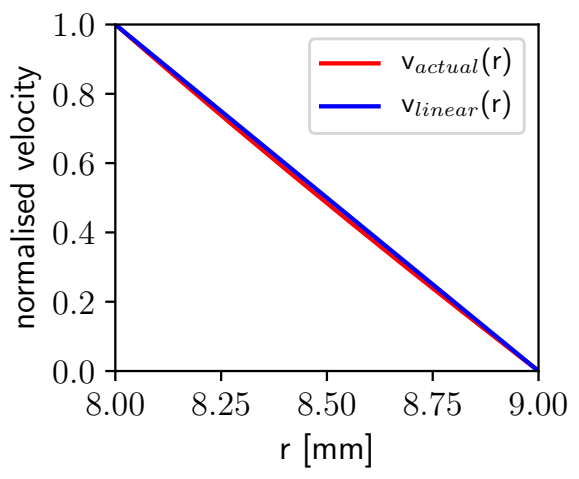

(a) $r_{i}=8 \mathrm{~mm}, r_{o}=9 \mathrm{~mm}$

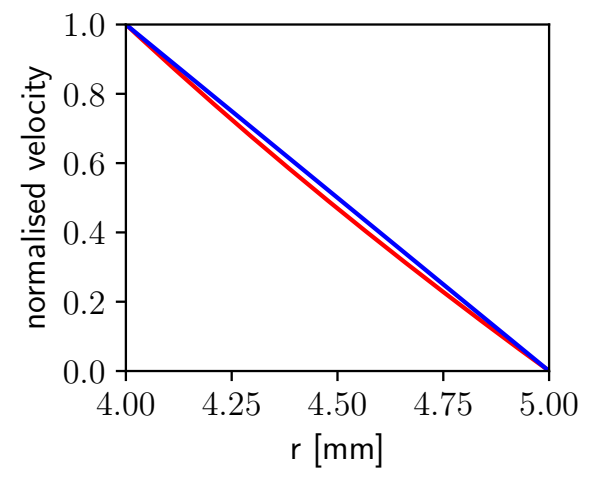

(b) $r_{i}=4 \mathrm{~mm}, r_{o}=5 \mathrm{~mm}$

Figure 2.7: Examples of TC geometry velocity profiles over the sample gap with different inner and outer wall radii. (a) Corresponds to the TC geometry used in this thesis to benchmark results, while (b) has the same dimensions as the cylindrical region of the novel geometry developed in this these. Equation (2.9) is compared with an assumed linear relationship for a homogeneous flow case.

\section{Cone and Plate}

The cone and plate design is attributed to Mooney and Ewart [16] from 1934. Since then it has arguably become the most favoured geometry for measuring normal forces of the material as well as having one of the best approximations to homogeneous flow.

The geometry comprises a stationary plate topped with a downwards facing cone, see Figure 2.8. The sample is placed in the angular section between these two surfaces and the shear is applied by rotating the cone. For derivations the geometry

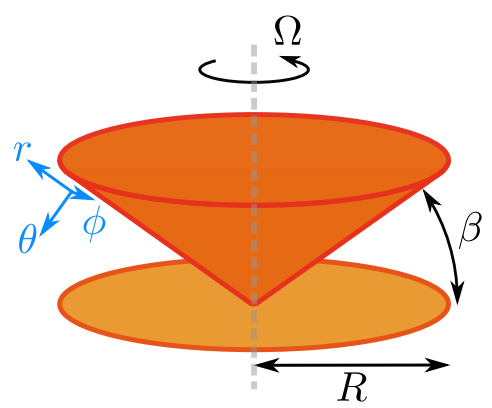

Figure 2.8: Cone and plate geometry, where a stationary plate and a rotating cone apply a shear to the sample trapped between them. 
is usually assumed to have spherical symmetry and the shape of the exposed end is ignored. Here the key dimension is $\beta$, the angle between the plate and the surface of the cone.

Stress varies with position, this time an angle $\theta$, across the sample width here as well. The ratio of the shear stress from the lower to upper wall is of the form

$$
\frac{\sigma_{\phi \theta}\left(\theta_{\text {cone }}\right)}{\sigma_{\phi \theta}\left(\theta_{\text {plate }}\right)}=\frac{1}{\sin ^{2}(\pi / 2-\beta)} \text {. }
$$

Similar to the narrow gap approximation, the cone and plate has a small angle approximation to allow the variation in the stress to be ignored. For an angle of $5^{\circ}$ this translates to a variation of less than $1 \%$ and for an angle of $10^{\circ}$ this translates to a variation of just over $3 \%$. For commercial rheometers it is accepted that the small angle approximation is valid for a variation of half a percent or less, equivalent to $4^{\circ}$.

Assuming this negligible variation, this leads us to a shear rate defined as constant over the gap and equal to $\dot{\gamma}(\theta)=\frac{\Omega}{\beta}$ where again $\Omega$ is the angular velocity of the rotating element (cone).

\subsubsection{Complex Materials}

Many interesting everyday materials do not conform to the distinct Hookean solid or Newtonian liquid behaviour that was introduced on page 1 , and explicitly defined in Section 2.1.1 by equations (2.1) and (2.2). Instead these Hookean and Newtonian descriptions form boundary conditions for 'complex materials'. These can exhibit both elastic and solid properties, often depending on the mechanical interactions and time scales, and are therefore referred to as viscoelastic. Note that non-Newtonian fluids do not obey the well known Navier-Stokes equations, but do not necessarily require viscoelastic properties.

Within the realm of viscoelasticity there are a variety of material behaviours that can be represented in the form of equation (2.2) with a non-constant viscosity. Time dependent viscosities $\eta(t)$ increase - thixotropy (i.e. paint) - or decrease antithixotropy - for a constant deformation, followed by a gradual recovery once stimuli is stopped. Shear thinning and shear thickening occur when the viscosity decreases or increases for increasing shear rate (Figure 2.9 shows the corresponding shear rate verse stress plots i.e. flow curves). Walking on custard is possible due to shear thickening, while shear thinning makes shampoo, tomato sauce, mayonnaise, and toothpaste spreadable. Elastic behaviour in fluids can also manifest itself in terms rod climbing or ductless siphons. ${ }^{5}$

\footnotetext{
${ }^{5} \mathrm{An}$ interested reader is recommended to look an the wonderful images of such phenomena col-
} 


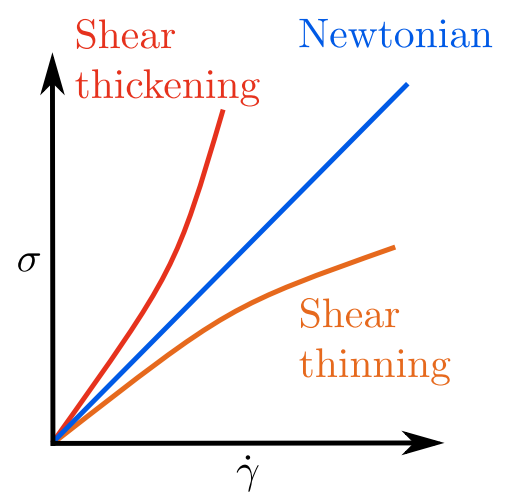

Figure 2.9: Example of the characteristic shapes of flow curves due to shear thickening and shear thinning compared to a Newtonian fluid.

\section{Shear-Banding}

Shear banding is a rheological phenomenon that a sample used in this research exhibits. The phenomena will be discussed here in general and the sample more specifically in Section 3.1.3.

The phenomenon expresses itself as the onset of a shear induce phase transition that results in the material partitioning into two bands or regions of differing viscosities / shear rates, between the stationary and moving wall, Figure 2.10a. This behaviour is seen in polymers and surfactant wormlike micelle (WLM) systems. Both materials are made up of long chains of flexible molecules which align in a nematic phase (in the high shear rate band) and in an isotropic entangled phase (in the low shear rate band). An in-depth introduction to the WLM systems for the interested reader can be found in Berret [18], Rothstein [19, and references therein].

Typically studies for WLM systems have been done in a Taylor-Couette geometry where the outer stationary wall has a lower shear rate band (similar in value to the shear rate prior to the onset of shear banding). A higher shear rate band is generated by the inner moving wall whose dynamics are thought to follow a lever rule

$$
\dot{\gamma}_{a}=(1-\alpha) \dot{\gamma}_{1}+\alpha \dot{\gamma}_{2}
$$

where $\alpha$ is the relative thickness of the band with the higher local shear rate $\dot{\gamma}_{2}$, and $(1-\alpha)$ and $\dot{\gamma}_{1}$ is lower local shear rate band.

The rheological signature for this manifests itself as a stress plateau shown in Figure $2.10 \mathrm{~b}$ where homogeneous flow is present before phenomena starts. For WLMs, shear banding has been extensively studied including using a variety of op-

lected in Rheological Phenomena in Focus Boger and Walters [17]. 
tical and visualisation techniques which physically demonstrate the banded structure. This will be discussed in Section 2.4.1.

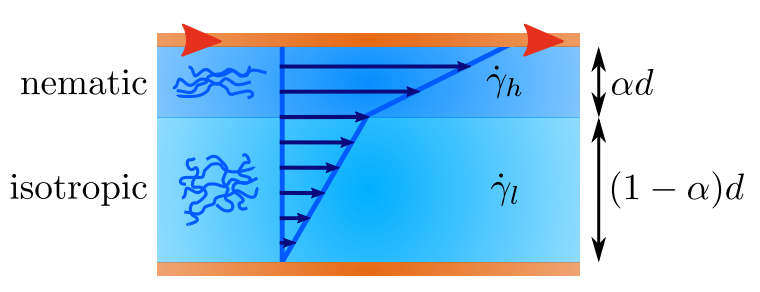

(a)

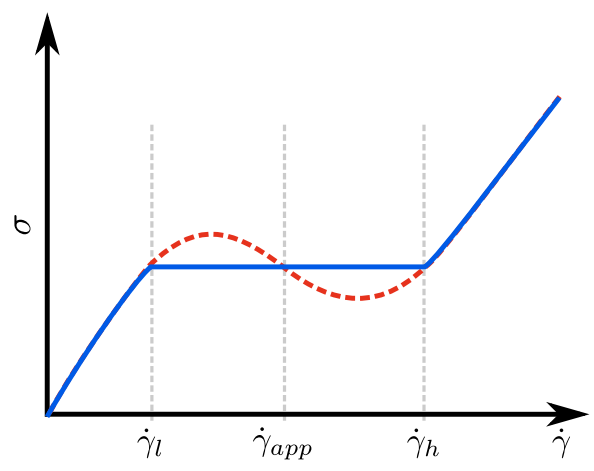

(b)

Figure 2.10: (a) The expected flow field for a shear banding material as defined by the lever rule, equation (2.11). (b) Schematic of a steady state flow curve that shows the characteristic stress plateau that implies the onset of the shear banding phenomenon. Solid line is the observed flow curve, while the dashed line is the constitutive relation assumed for wormlike micelles.

\subsection{Nuclear Magnetic Resonance}

NMR is a natural phenomena which was discovered in first half of the last century. It then developed as a scientific technique as a result of the groundbreaking measurements taken independently by Bloch [20] and Purcell et al. [21] in 1946. Since then the field has exploded. Through advancements in NMR theory and experimentation, the technique has shown to be applicable for a variety of research, industrial, and diagnosis studies; from chemical spectra analysis to MRIs (magnetic resonance imaging) in hospitals.

The study of NMR hinges on detecting nuclear spin precession. As such the theory relies fundamentally on quantum mechanics. Accepting that nuclear spin results in a magnetic moment, a material can be generalised in terms of a local magnetisation of a collection of nuclear magnetic moments. Using this, a semiclassical description, relevant to the research of this thesis, is briefly introduced below. A more complete quantum mechanical description can be found in most introductory books to NMR [e.g. 22-24]. 


\subsubsection{Larmor frequency and Bloch-Torrey Equations}

A basic principle behind NMR experimentation is subjecting matter, in particular the inherent spins of the nuclei, to magnetic fields and observing their behaviour.

Spins, and their corresponding moments, establish an equilibrium where they are polarised when placed in an external static magnetic field. This polarising field is labelled $B_{0}$ and its direction conventionally defines the $z$ axis in the Cartesian reference frame of the system. Considering a macroscopic sample, the number of spins is sufficiently large that the cumulative effect of the individual moments creates a net or local magnetisation $M_{0}$ vector along $z$ whose magnitude is proportional to the magnetic susceptibility $\chi$ and $B_{0}$

$$
M_{0}=\frac{N \gamma^{2} \hbar^{2}}{4 k_{B} T} B_{0}=\chi B_{0} .
$$

Here the expression is evaluated for a ${ }^{1} \mathrm{H}$ proton spin $1 / 2$ system, with $N$ being the number of local nuclei, $k_{B}$ the Boltzmann constant, and $\hbar$ the reduced Planck's constant $[23]^{6}$. The remaining term, $\gamma$, is referred to as the gyromagnetic ratio and is characteristic for each atomic nuclei ${ }^{7}$.

The local magnetisations can be thought of as magnetic densities which result from density of spins in the sample and their environment. Treating the problem semi-classically, the change in momentum of the local magnetisation $M_{0}$ in position $\boldsymbol{r}$ can be described by

$$
\frac{d \boldsymbol{M}(\boldsymbol{r}, t)}{d t}=\gamma \boldsymbol{M}(\boldsymbol{r}, t) \times \boldsymbol{B}_{\mathbf{0}}
$$

as $B_{0}$ exerts a torque on the magnetisation. The solution to this reveals the magnetisation freely precessing about the $z$ axis at a frequency

$$
\omega_{L}=\gamma B_{0}
$$

the all important Larmor frequency [20, 24]. Note that it is the Larmor equation which translates a magnetic field magnitude into a frequency for a specific nuclei ${ }^{8}$.

NMR also requires a second perpendicular field $B_{1}$, which oscillates at the Larmor frequency. It is from this that the 'resonance' in NMR becomes apparent; tuning the perpendicular $B_{1}$ signal to the natural Larmor frequency of a specific magnetic system of interest (e.g. ${ }^{1} \mathrm{H}$ ) allows the practitioner to distinguish different chemical resonance frequencies to select out the components due to nuclei of interest $\left({ }^{1} \mathrm{H}\right.$ in this example).

\footnotetext{
${ }^{6}$ Chapters 1 and 2 describe the derivations for equations (2.12) - (2.14).

${ }^{7}$ For protons this is $\gamma=267.5 \times 10^{6} \mathrm{rad} / \mathrm{sT}$.

${ }^{8}$ Many NMR systems may be referred to by their proton frequency (e.g. $400 \mathrm{MHz}$ ) rather than their magnetic field strength (e.g. 9.4 T).
} 
For further consideration of the local magnetisation $M_{0}$, and in particular its evolution in time $M(t)$, it is intuitive to work in a reference frame rotating with respect to $B_{1}$ around $z$. In the rotating frame, if $B_{1}$ is tuned to $\omega_{L}$, then $M_{0}$ and $B_{1}$ are stationary so do not precess, while the apparent $B_{0}$ field vanishes. The evolution can be described by the Bloch-Torrey equation, dependant on position

$$
\frac{\partial \boldsymbol{M}(\boldsymbol{r}, t)}{\partial t}=\gamma \boldsymbol{M} \times \boldsymbol{B}-\left(\frac{M_{x}}{T_{2}}, \frac{M_{y}}{T_{2}}, \frac{M_{z}-M_{0}}{T_{1}}\right)+\nabla \cdot \boldsymbol{D} \nabla\left(\boldsymbol{M}-\boldsymbol{M}_{0}\right) .
$$

The first term in the equation is the familiar contribution of the torque exerted by the magnetic field on the magnetisation, but this time with the added components from $B_{1}$. The second is the contribution from the relaxation process of the spins in the longitudinal $T_{1}$ and transverse $T_{2}$ directions (decay of magnetisation back to equilibrium after excitement and loss of coherence of magnetisation in transverse plane respectively), which will be further discussed in Section 2.2.3. At this point we have the Bloch equations [20], which were generalised by Torrey to account for molecular diffusion [25] described by $\boldsymbol{D}$, the diffusion tensor.

It is the Bloch-Torrey equation and its solutions that form the basis for understanding many basic NMR experimental features.

\subsubsection{NMR Excitation and Detection}

\section{Radio Frequency Pulse}

The $B_{1}$ perpendicular field is commonly referred to as an RF pulse (as the Larmor frequency that it is chosen to oscillate at is in the radio frequency part of the spectrum). The pulse is typically applied for a time on the order of $\mu s$. On these time scales influences from the relaxation and diffusion terms in the Bloch-Torrey can be neglected, leaving only the first term which now describes the torque from $B_{1}$ reorienting the magnetisation through a tip angle $\phi$ determined by the time, $t_{p}$, over which $B_{1}$ was applied, $\phi=\gamma B_{1} t_{p}$.

The initial excitation of the magnetisation is in general done with a $\pi / 2$ pulse to transfer the magnetisation completely into the transverse plane and let it decay back up to equilibrium, governed by relaxation and diffusion after the RF pulse. The response creates a change in flux which induces a detectable voltage in the receiver coil inside the magnet system, the NMR signal.

\section{Principles of NMR experiment}

Many NMR experiments involve initially setting the samples magnetisation in a $B_{0}$ field, then applying a $\pi / 2$ excitation to disturb it from equilibrium. This magneti- 
sation is then manipulated by its environment and a sequence of other RF pulses and gradients (spatially encoding magnetic fields, to be discussed in Section 2.2.4) under which spatially local pockets of magnetisation precess at local Larmor frequencies. The magnetisation is left to lose phase coherence while also returning to equilibrium. During this process specific parts of the magnetisation can be purposely dephased and rephased. Under time reversal this creates a signal 'echo' which, once detected and analysed, conveys information on the sample environment (such as spin location, which can be related back to a semi-classical idea stated in the below section).

A specific string of pulse, gradients, and timings are called a pulse sequence or pulse program. The versatility of NMR comes from the number of permutations of pulse sequences which allows a skillful practitioner to do spectroscopy (obtain a chemical spectra of a molecule's unique magnetic environment), relaxometry (calculate $T_{1}$ and $T_{2}$ ), magnetic resonance imaging (density map of protons), diffusion and velocimetry (molecular motion and pore structure), or some combinations of these in a single experiment. Physically parts of this are enabled by the NMR instrumentation using a synthesiser - which compares the mathematically complex signal transmitted into a sample and the signal acquired - to extract phase information. This is what sets NMR apart from other light based techniques like scattering and x-ray, which can only focus on intensity.

\subsubsection{Relaxometry}

The second term in the Bloch-Torrey equation (2.15) contains the influence of the relaxation times $T_{1}$ and $T_{2}$ on the local magnetisation. These two mechanisms are further explained below. The study of the relaxation times $T_{1}$ and $T_{2}$ is useful for timing NMR experiments but can additionally yield information on soft matter systems as will be briefly mentioned in Section 2.3.1. The pulse sequences for finding the relaxation times are briefly described but can be pictorially seen in Callaghan [22, Section 2.6]. The term spin is used but may be thought of as individual nuclei magnetic moments.

\section{Longitudinal $\left(T_{1}\right)$ Relaxation Time}

$T_{1}$ is called the longitudinal or spin-lattice relaxation time. Energy is put into the spin system by the $\pi / 2$ excitation pulse to rotate the magnetisation into the $x y$ plane. This energy later dissipates, with the characteristic relaxation time of $T_{1}$, through the interactions of individual spins with their surroundings (lattice) re- 
turning the local magnetisation to equilibrium alignment with the $B_{0}$ field. Isolating the $T_{1}$ behaviour from equation (2.15), this process will be described by the differential equation

$$
\frac{d M_{z}}{d t}=-\frac{M_{z}-M_{0}}{T_{1}}
$$

in this case with a $\pi / 2$ excitation occurring at $t=0$. The solving the differential equation can give a solution in the form

$$
M_{z}(t)=M_{z}(0) e^{-t / T_{1}}+M_{0}\left(1-e^{-t / T_{1}}\right) .
$$

Physically $T_{1}$ can be measured through an inversion recovery process. An initial $\pi$ excitation is used to flip the magnetisation to anti-parallel with the $z$ axis. A time $\tau$ is allowed to elapse in which the magnetisation begins to return to equilibrium under $T_{1}$, then a $\pi / 2$ pulse rotates the magnetisation into the $x y$ plane where its magnitude can be detected. This process is repeated for a range different times in which the magnetisation has had a chance to undergo $T_{1}$ relaxation. From the initial $\pi$ pulse equation (2.16) simplifies to

$$
M(t)=M_{0}\left(1-2 e^{-t / T_{1}}\right)
$$

and a value of the spin-lattice relaxation can be extracted from plotting the inversion recovery data to such a curve. It is critical to know this value when running successive scans of an NMR experiment as residual magnetisation would effect the results of future scans. In the common case of sequences with $\pi / 2$ pulses spin recovery time is $5 \times T_{1}$ by which equilibrium will have been restored.

\section{Transverse $\left(T_{2}\right)$ Relaxation Time}

$T_{2}$ is the transverse or spin-spin relaxation time and is solely concerned with the behaviour in the $x y$ plane. Here no energy is added or removed from the system but exchanged between neighbouring spins as each experiences a slightly different magnetic environment due to each others moments. As one spin gains energy and the other loses it, their precession frequencies are either sped up or slowed down. This begins to dephase the coherent local magnetisation. These energy exchanges are random, so the process is irreversible and the signal is lost. Isolating the $T_{2}$ behaviour from equation (2.15) shows that this process will be described by the differential equation

$$
\frac{d M_{x, y}}{d t}=-\frac{M_{x, y}}{T_{2}}
$$

with solution

$$
M(t)=M_{x, y}(0) e^{-t / T_{2}}
$$


A Carr-Purcell-Meiboom-Gill (CPMG) pulse sequence [21, 26] is used to measure $T_{2}$. The original magnetisation $M_{x, y}(0)$, resulting from a $\pi / 2$ excitation, will begin to lose coherence and dephase due to spin-spin transferrals. After a time $\tau$, an inverting $\pi$ pulse flips the $x y$ plane magnetisation so after the same time $\tau$, the nonrandom dephasing will have rephased into an echo. Its height is smaller than after the initial pulse as the random $T_{2}$ effects can not be rephased. Therefore the decay in the $x y$ plane signal of the echo train is governed by $T_{2}$. It is measured through continuation of the experiment with successive $\pi$ pulses to allow for dephasing and rephasing into new echos, which decay in magnitude by equation (2.19).

$T_{2}$ is limited by $T_{1}\left(T_{1} \geq T_{2}\right)$, as the magnetisation is decaying with $T_{1}$ back to thermal equilibrium. Therefore spin-spin relaxation will cause a decay faster than spin-lattice. Both relaxation times are important as they act as limiting factors of the total length of an NMR experiment after which there are significant reductions in signal-to-noise and eventual complete loss of signal. $T_{2}$ relaxation is impacting when spins are in $x y$ plane and $T_{1}$ when they align in $z . T_{2}$ is of particular focus when using RARE based imaging experiments (see Section 2.2.4) as their pulse sequences are very similar in structure to CPMG trains.

\subsubsection{Magnetic Field Gradients}

When Rheo-NMR is introduced later in Section 2.3, examples are given of different types of NMR experiments that can be applied to study the rheological behaviour of matter. Specifically, the aim of NMR studies in this thesis is to observe material velocities within a shear geometry. To achieve this it is necessary to encode the material for both position and displacement (and hence velocity). There are two methods which are used in this thesis to achieve this: a 1D image (or profile) and a $2 \mathrm{D}$ image. Both are sensitive to local displacements, so velocity information can be overlaid on the image dimension(s) (i.e. making a velocity map). The two processes, velocity encoding and imaging, involve measuring position in terms of material distribution and dynamics: imaging requires position measured once, while velocity (and diffusion) require measuring a position twice and extracting the displacement. For a further interest in the use of magnetic field gradients the reader is directed to Callaghan [27].

\section{Velocimetry}

Velocimetry, PGSE (Pulsed Gradient Spin-Echo) NMR, propagator (velocity probability distribution) encoding, transversing q-space (reciprocal displacement space), 
or translational dynamics NMR, are all terms which may be given to the processes for obtaining velocity / flow information with NMR [22, and reference therein].

The core of velocimetry experiments is to first impart a local Larmor frequency distribution inside the sample. Spins (or local magnetisation vectors) then acquire a specific phase over time, characteristic for their position in space. This requires a non-static non-homogeneous background magnetic field (in addition to the static ideally homogeneous $B_{0}$ ) which instead has a variation in space. The NMR instrumentation commonly applies a non-homogeneous field, using gradient coils applying gradient pulses, aptly named as the first derivative of the field (including the homogeneous $B_{0}$ ) gives a constant gradient $\mathbf{G}$ (i.e. $\mathbf{G}=\nabla B_{z}{ }^{9}$ ).

If spins are experiencing slightly different, but well defined, magnetic environments then they will precess at slightly different Larmor frequencies when compared to their neighbours. The frequencies being determined by equation (2.14). Having well defined magnetic environments is essential as this makes it applicable for meaningful spatial encoding. Position dependence of local precession is

$$
\omega(\mathbf{r}, t)=\gamma B(\mathbf{r}, t)=\gamma\left(B_{0}+\mathbf{r} \cdot \mathbf{G}(t)\right)
$$

where the time dependence comes solely from $\mathbf{G}(t)$ as it is assumed that the material elements are fixed during the gradient pulse. Similarly this can be integrated over a time during which the gradient may be turned on and off and the encoding considered instead as imparting a phase pattern on the spins that is a function of position

$$
\begin{aligned}
\int_{0}^{t^{\prime}} \omega(\mathbf{r}, t) d t & =\int_{0}^{t^{\prime}} \gamma\left(B_{0}+\mathbf{r} \cdot \mathbf{G}(t)\right) d t \\
& =\phi\left(\mathbf{r}, t^{\prime}\right) .
\end{aligned}
$$

As such the gradients can be thought of as winding up the phase of excited spins in a helical shape along the direction of the field variation [see 28, Figure 2] . This twisted pattern remains as the gradient is turned off and the entire system precesses at the Larmor frequency determined by the static $B_{0}$ field.

To measure displacement a gradient is applied a time $\Delta$ after the start of the initial gradient achieving opposite polarity. Simply swapping the sign of second gradient produces a gradient echo when the spins all rephase, $\Delta \phi=0$, while instead applying a $\pi$ RF pulse (inverting the sign of the phases) in-between two gradient pulses of the same sign gives a spin echo. Suppose a molecule containing a spin

\footnotetext{
${ }^{9}$ The assumption in labelling the total background field $B_{z}$ is that the static background field $B_{0}$ is considerably greater than the gradient field. Hence the $B_{x}$ and $B_{y}$ components of the total background field, that must exist due to Maxwell's laws, are relatively negligible [27].
} 
has moved in the time $\Delta$, it will not see the identical environment to unwind its phase pattern and a residual phase will be left. In the spin echo case the residual phase is

$$
\begin{aligned}
\Delta \phi\left(\mathbf{r}, t^{\prime}\right) & =\int_{0}^{\tau} \gamma\left(B_{0}+\mathbf{r}_{\mathbf{1}} \cdot \mathbf{G}_{\mathbf{1}}(t)\right) d t-\int_{\tau}^{2 \tau} \gamma\left(B_{0}+\mathbf{r}_{\mathbf{2}} \cdot \mathbf{G}_{\mathbf{2}}(t)\right) d t \\
& =\int_{0}^{\tau} \gamma \mathbf{r}_{\mathbf{1}} \cdot \mathbf{G}_{\mathbf{1}}(t) d t-\int_{\tau}^{2 \tau} \gamma \mathbf{r}_{\mathbf{2}} \cdot \mathbf{G}_{\mathbf{2}}(t) d t \\
& =\gamma \mathbf{r}_{\mathbf{1}} \cdot \mathbf{g}_{\mathbf{1}} \delta-\gamma \mathbf{r}_{\mathbf{2}} \cdot \mathbf{g}_{\mathbf{2}} \delta
\end{aligned}
$$

where $\delta$ is the time that the gradients of magnitude $g_{1}$ and $g_{2}$ are applied and $\tau$ is the time when the $\pi$ RF pulse occurs. Setting the gradient amplitude equal $g_{1}=g_{2}=g$

$$
\begin{aligned}
\Delta \phi\left(\mathbf{r}, t^{\prime}\right) & =\gamma \delta \mathbf{g} \cdot\left(\mathbf{r}_{1}-\mathbf{r}_{2}\right) \\
& =\gamma \delta g(v \Delta) \\
& =\Delta \phi(\delta g, v \Delta)
\end{aligned}
$$

with $v \Delta$ being the velocity component in the displacement encoding direction set by the gradient coils multiplied by the displacement time $\Delta$. This makes the residual phase proportional to the parameter we which to extract, the velocity. Note that $\delta g$ is proportional to a vector $\boldsymbol{q}=\gamma \delta \boldsymbol{g} / 2 \pi$ which characterises a reciprocal displacement space (the q-space eluded to earlier). Fourier transforming q-space into displacement space gives a displacement probability distribution called the propagator $P(Z, \Delta)$. The peak of this distribution is the mean velocity.

Velocimetry allows for the extraction of velocity data. However to get the velocity 'map' that is desired for this work the information needs to be spatially localised. The velocity sensitive component needs to be followed up by an imaging experiment.

\section{Imaging}

Magnetic Resonance Imaging (MRI) is one of the most widely recognised NMR applications due to its use in hospitals as a diagnostic and monitoring tool. Like velocimetry it uses magnetic field gradients, however it only requires imprinting position information on the spins to link them with a location in absolute space.

For 1D imaging, and the first dimension of 2D imaging, frequency encoding is one method used (and the particular one utilised in this research). As mentioned previously, subjecting the sample to a gradient places the spins in different magnetic environments and 'labels' them with a precession frequency governed by 
equation (2.20). The sample in this state can be described as a spectra of precession frequencies weighted by the density of spins in each Larmor frequency environment. With frequency encoding, the NMR signal is acquired while the gradient is on, collecting data in the time domain (as the inverse Fourier Transform of the spectra). The time domain is linearly related to an imaging space, $\mathrm{k}$-space, define by

$$
\boldsymbol{k}=\frac{\gamma \boldsymbol{g} t}{2 \pi} .
$$

$\boldsymbol{k}$ is reciprocal for position in real space so a further Fourier Transform translates k-space data into a plot in real space with each point weighted by the density of spins. This completes the circuit of domains and Fourier Transforms as this image is simply the initial Larmor frequency spectra but in terms absolute position in space. The first direction in imaging is commonly referred to as the read direction, and gradients used to do this type of k-space encoding are called read gradients.

As the above approach transversed the imaging k-space by stepping through $t$ with a fixed gradient (as other terms in equation (2.23) are constant) a different method must be used to transverse $k$-space in an orthogonal direction to create a 2D image. This can be done by iterating different gradient strengths in the second direction applied for a fixed time $t$. k-space is stepped though using gradients that impart a different winding or phase on the spins for each value of $\boldsymbol{g}$. This uses the effect of the gradients with respect to equation (2.21) as apposed to equation (2.20), the first dimension imaging approach. As such this direction is called the phase direction and the type of encoding is phase encoding. A Fourier Transform in both directions on k-space will produce a 2D spin density map of the sample.

A third dimension in imaging space is termed the slice direction. For imaging directions that do not undergo frequency or phase encoding (phase and slice for 1D and just slice for 2D) slice selection is used to determine a thickness of sample that the image is averaged over. This technique utilises gradients, in the non-imaging directions, pairing them with a simultaneous $\pi$ RF pulse with a set bandwidth. Only the spins with a Larmor frequency defined by (2.20) that fall within the bandwidth of the RF pulse are effected by it and their phases inverted. Spins with Larmor frequency outside the bandwidth continue to dephase and magnetisation from these spins are lost, leaving the magnetisation just from within the slice in space.

\section{Pulse Sequences}

The specific pulse sequences used by this thesis are a PGSE-RARE experiment (2D velocimetry using Rapid Acquisition with Relaxation Enhancement (RARE) imaging) and a 1D velocimetry with double slice selection experiment, shown in Figures 


\subsection{1 and 2.12 respectively ${ }^{10}$.}

The PGSE-RARE experiment [29-31] encodes for velocity using a Pulse Gradient Spin-Echo sequence shown in the first portion of the pulse sequence. This is followed by a RARE imaging sequence [32] which transverses k-space in the phase direction through successive echos looping the phase gradients so a complete image can be collected off a single excitation (saving time). When processed the result is a $2 \mathrm{D}$ image of a sample cross section, coloured to show direction and magnitude of flow. Note that velocity can only be encoded for in one direction so to get a complete 3D flow field three velocity maps would be required. The benefit of having a $2 \mathrm{D}$ velocity map is seeing not only the velocity profile but its evolution in space.

A 1D velocimetry experiment [33] was also used to obtain simple velocity profiles across the sample. In particular the experiment was used for the case where the second imaging direction had no variation and therefore did not add anything to the results. This begins with a PGSTE component, using a STimulated Echo rather than a Spin Echo ${ }^{11}$.

\subsubsection{The NMR Spectrometer}

Experiments conducted as part of this thesis employed the use of a Bruker wide vertical bore superconducting NMR spectrometer, seen in Figure 2.13. The Larmor frequency of ${ }^{1} \mathrm{H}$ for this magnet system is $400 \mathrm{MHz}$ (equivalent to a magnetic field strength of $9.4 \mathrm{~T}$ ). It comprises a superconducting coil to generate the static $B_{0}$ field. An RF coil with a $25 \mathrm{~mm}$ internal diameter was used for all experiments (shown 2.14a bottom left) and is inserted into the center of the $B_{0}$ field coil with a probe stand. The samples and geometries of interest are housed inside the RF coil. Inside the magnet system, around the RF coil, is a Micro2.5 system containing three orthogonal gradient coils, to allow for both spatial and displacement encoding in all three dimensions. These define $x, y, z$ in the lab frame. Experiments were run using TopSpin and ParaVision software from Bruker.

\footnotetext{
${ }^{10}$ Note that the RF pulses are depicted two different ways, as hard lines or soft sinc functions. Soft RF pulses are ripple-like in the time domain of the pulse sequence but under Fourier Transform they approximate a hard hat function in the frequency domain with a fixed bandwidth which is useful for slice selection.

${ }^{11}$ An obvious difference between the two echoes are that two $\pi / 2$ pulses invert the displacement encoding spins with STE rather than one $\pi$ with SE.
} 


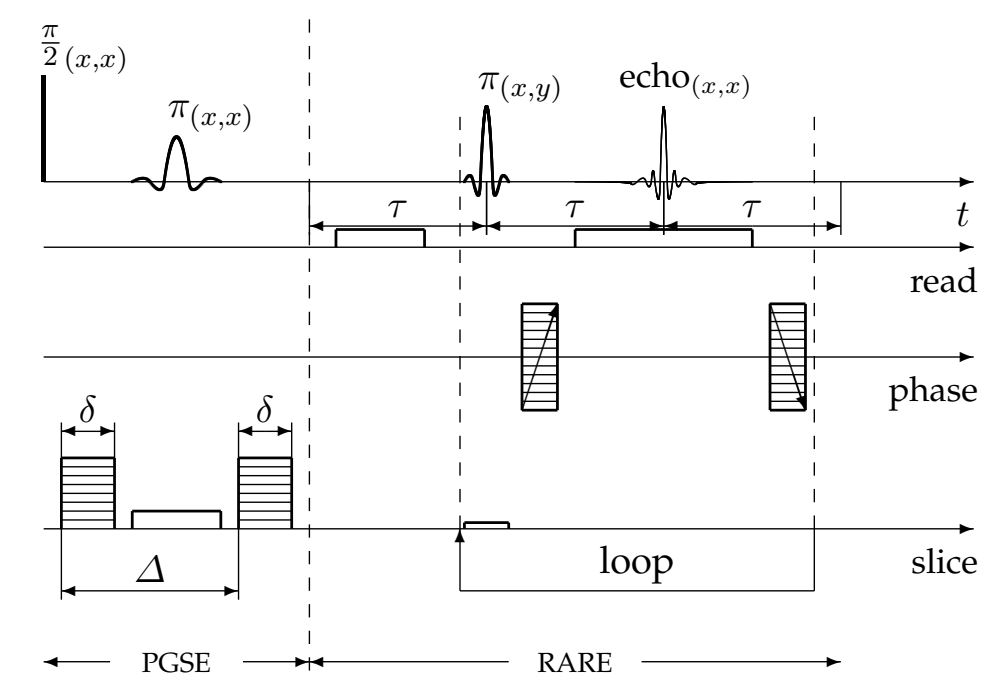

Figure 2.11: PGSE-RARE pulse sequence used to record 2D velocity maps. It utilises both displacement and spatial encoding methods.

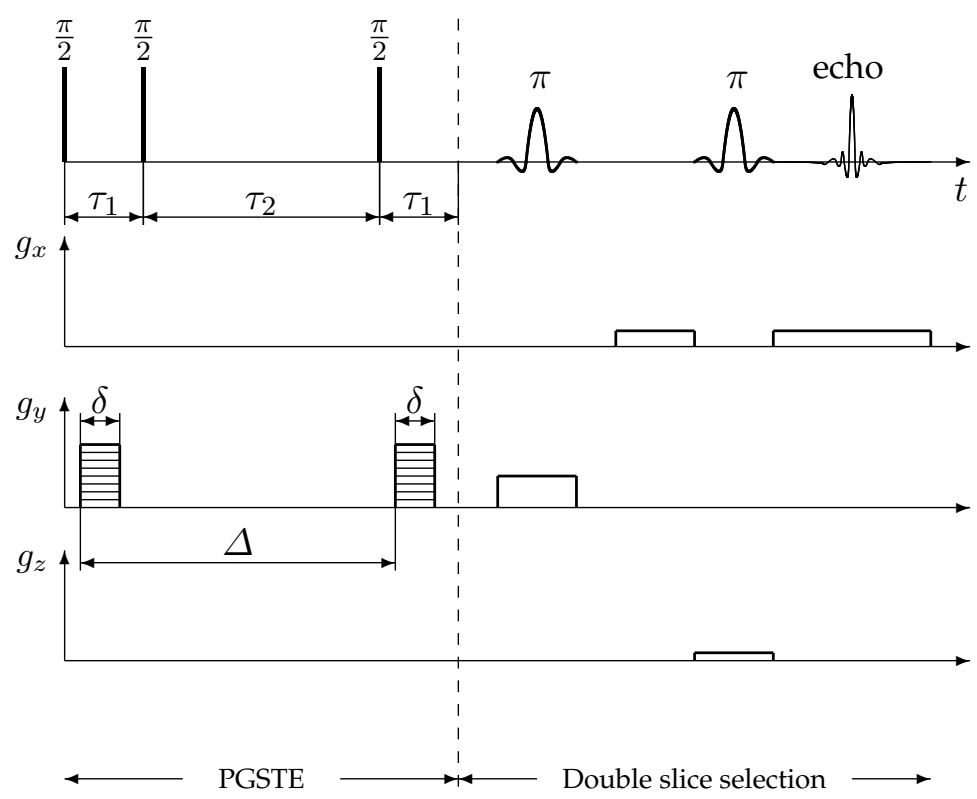

Figure 2.12: 1D double slice selection pulse sequence used to record $1 \mathrm{D}$ velocity profiles. 


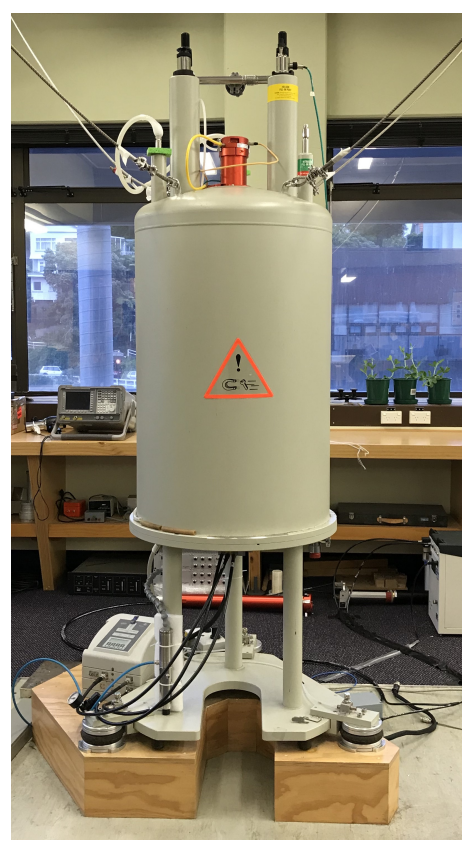

Figure 2.13: Bruker $400 \mathrm{MHz}$ NMR magnet used for this thesis. Console and other peripherals not shown.

\subsection{Rheo-NMR}

As mentioned previously, NMR is one technique, among many, which aims to contribute to rheological studies by probing microscopic features of samples under deformation. A brief history of the combined technique of rheo-NMR and range of hardware used is introduced.

\subsubsection{Enhancing Rheological Studies with NMR}

Complex material deformation has been explored with NMR since at least the 1980s [34-36]. While not mechanically shearing the sample in the classical sense of rheometry, Martins et al. (1986) used spectroscopy (in particular, a relaxation of chemical shift due to director realignment) to extract viscoelastic coefficient for a nematic polymer system. Here the deformation was applied by disturbing the alignment of the macromolecules with the strong $B_{0}$ field through their diamagnetic anisotropy characteristic. Vera et al. (1986) and Lacelle et al. (1991), again with spectroscopy, sheared samples in Taylor-Couette geometries to study flow patterns / mixing. However the coining of the term rheo-NMR can perhaps be credited to Nakatani and colleagues from their 1990 paper on chain deformation in sheared polymer fluids using line broadening of a NMR proton spectra: "Rheo-NMR, the 
use of nuclear magnetic resonance to monitor the behaviour of entangled polymers subjected to shear" [37]. This paper specifically discussed a cone and plate rheometer design (with cone angles of $4^{\circ}$ and $8^{\circ}$ ) rotating inside a NMR system, and includes an awareness of the benefits and challenges with combining these two experimental techniques.

In the past three decades since the late 1980s, the field of rheo-NMR has expanded in both the NMR and rheological tools used and the types of samples studied. A progressing summary of this development can be found in reviews done by Callaghan in 1999 [38], 2006 [39], and 2012 [40].

Rheo-NMR aims to enrich rheological studies of materials by providing a method to visualise the flow field generated by deformation devices, to spatially resolve the dynamics of samples, and to probe molecular organisation and order. This has the potential to better the understanding of complex material systems and improve rheological models, important to many industries that utilise such materials. Compared to other rheo- visualisation techniques NMR provides a unique contribution in its variety of pre-existing tools to study soft matter because it can probe behaviour / properties on a wide range (up to 10 orders of magnitude) of distance and time scales Callaghan [see 38, Figure 13]. Spectroscopy can be linked to order parameters and orientation [41], relaxometry has been shown to relate to stiffness [42], while diffusion distributions can tell us about the droplet sizes for emulsions [43]. It does this without the need for tracer or scattering particles, or a transparent sample, with the flexibility of full 3D spatial mapping and velocity encoding in any direction.

\subsubsection{Types of Hardware and Set Up}

The term rheo-NMR is additionally used to reference specific hardware developed for studying materials under mechanical deformation in conjunction with an NMR system.

Historically Bruker has distributed strain controlled shear drive shafts developed by Callaghan and colleagues and first commercialised by Magritek, for this purpose. These comprise a shear geometry (usually Taylor-Couette or cone and plate), a drive shaft, a stepper motor and optical encoder, and a control unit with a web interface to control the shear rate applied.

Home built drive shafts appear in rheo-NMR literature as well. Typically these are just to apply the mechanical deformation to the sample inside a magnet system so its dynamics can be studied. However there have been attempts to fully integrate rheometry into NMR experimentation by incorporating drive shafts with 
torque sensors [see 4,44]. This is a powerful tool as it allows for simultaneous measurements of material properties in conjunction with collecting a flow curve describing the exact same environment. Others have taken the reverse approach, using permanent magnet systems around a rheometer [45] with promising results. This low field rheo-NMR apparatus, detailed by Ratzsch et al. aims to achieve a comparable range and resolution as obtainable with separate commercially available NMR and rheometer devices.

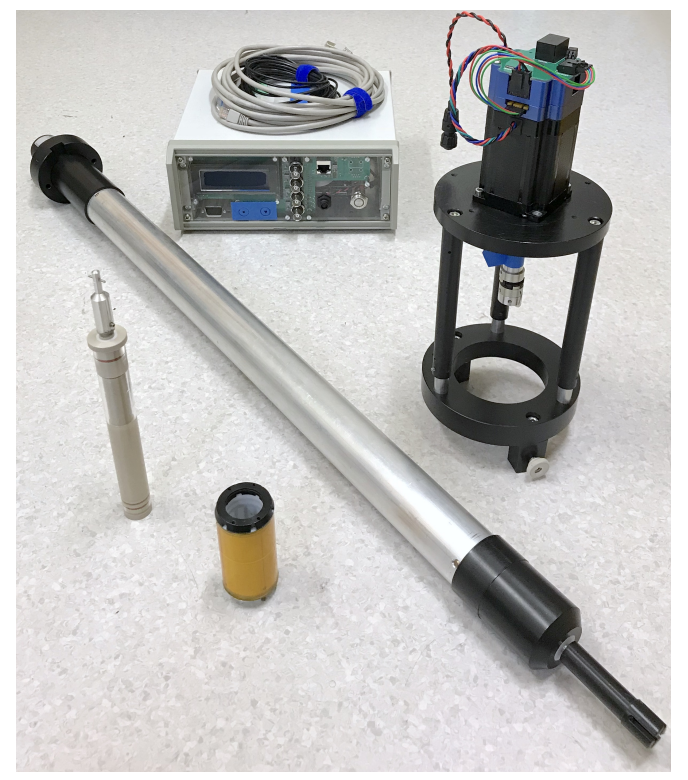

(a)

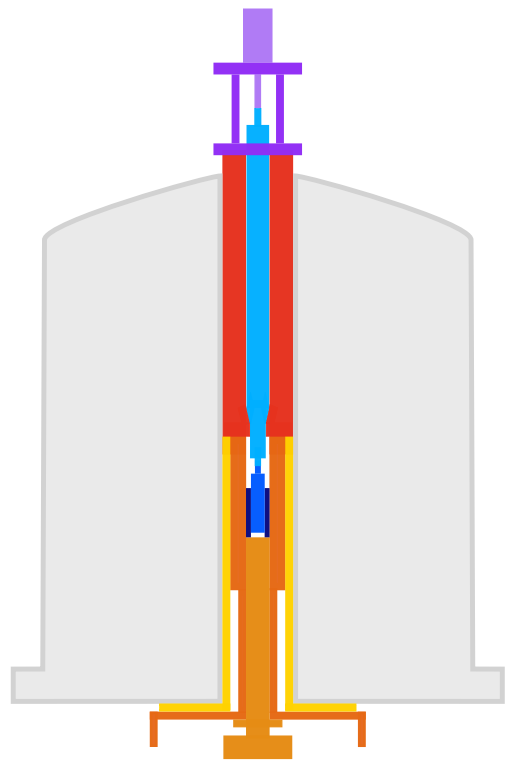

(b)

Figure 2.14: Components of Rheo-NMR kit (and RF coil) used in this thesis and how they are mounted in the magnet system (a,b respectively). This includes: a Magritek built drive shaft and motor stand (center / right, light blue / purple), a benchtop controller unit (top), a TC shear geometry (left, blue), an RF coil (bottom, navy). The coil is mounted on a probe (gold) to raise it to the correct height with respect to the magnet coil (not shown) and the imaging gradients (orange). The drive shaft is clamped to the upper shim barrel (red) which sits above the room temperature shims (yellow).

The rheo-NMR set up for the work presented in these thesis at times is a combination of (a) a Magritek style drive shaft and motor stand, and (b) a stepper motor, shear geometry, control unit, and software (in further development to [4]). The components are displayed in Fig. 2.14, with $2.14 \mathrm{~b}$ demonstrating the integration between the driveshaft and $400 \mathrm{MHz}$ NMR magnet. The TC shear geometry is positioned inside the RF coil with the bulk of the fluid area occupying the center (most 
homogeneous field) of the RF coil. It is coupled with a ' $\mathrm{T}$ ' shaped pin to the drive shaft which itself is clamped to the upper shim barrel to hold in place. A stepper motor and optical encoder in a feedback loop apply the motion to the shaft and are controlled through a benchtop controller unit. This is programmed via a PC web interface with a command line. The frequency range achievable in either clockwise or anti-clockwise rotation $0.01-10 \mathrm{~Hz}$ with a $0.01 \mathrm{~Hz}$ resolution.

\subsection{Case Study: Planar Shear Geometry}

As mentioned previously, there are an assortment of shear deformation geometries used in rheometry to study the mechanical behaviour of materials. Here the motivation and preliminary development of a novel shear geometry is discussed. This is of interest as it establishes the basis for the research described in the rest of thesis.

\subsubsection{Influence of Curvature in Taylor-Couette Geometry}

A notable stress (pun intended) is put on the design of many shear geometries used in rheometry: that they impart a homogeneous deformation. Approximating the shear environment as one that creates the simplest deformation (uniform stress) is beneficial when studying the complex materials typically of interest in rheology, as an uncomplicated limiting case. A theoretical description of the mechanical response is easier to realise, analysis is simpler to conduct, and the behaviour observed is characteristic of the material properties rather than the potentially more complicated fluid dynamics environment. The level to which the homogeneous approximation is valid depends on the specific geometry and relative dimensions.

For the Taylor-Couette geometry, prominent in both rheometry and rheo-NMR, a mathematical model was introduced in Section 2.1.2. When $r_{i} \approx r_{o}$, in a Newtonian liquid case, the model simplifies and the geometry is able to be considered the equivalent environment to a two infinite parallel plate arrangement (i.e. produce planar homogeneous flow). Physically this can be approached when the concentric cylinder's radii are large and as close to equal as still leaves a physical sample gap. For NMR adaptions this is harder to realise due to dimension restrictions, however it is plausible for benchtop rheometer.

To maintain the inherent cylindrical symmetry of the forces acting on the sample it is necessary to have a vertical bore NMR spectrometer. The wide vertical bore superconducting magnet used for this research can account for object diameters of up to $25-30 \mathrm{~mm}$. Superwide bore magnets only improve this by just over a factor of two. The narrowness of gap size $(\geq 1 \mathrm{~mm})$, and hence inner radius and 
$\kappa$ (equation (2.7)), are additionally limited by signal-to-noise considerations ${ }^{12}$. For example, the rheo-NMR Taylor-Couette geometry shown Figure 2.14a has an inner radius of $8 \mathrm{~mm}$ and an outer of $9 \mathrm{~mm}$, translating to $\kappa=0.89$, a stress variation difference of $21 \%$. Consequently the stress variation implicit in the Taylor-Couette geometry is more pronounced in rheo-NMR applications than those used in rheometry.

The influence of stress variation in the Taylor-Couette geometry was explored by Fardin et al. [46] and more specifically in terms of the geometric factor by Brox et al. [3]. Brox et al. looked at a collection of custom built geometries with $\kappa$ ranging from 0.67 to 0.96 , including a set of geometries of different outer radii with a constant $\kappa$ of 0.89 . A shear banding wormlike micelle solution similar to that described in Section 2.1.3 was tested using NMR-velocimetry. For geometries with identical $\kappa$ the onset of shear banding occurred at a set shear rate. However the onset of shear banding varies as a function of shear rate inhomogeneity, shifting to smaller shear rates for less ideal geometries. The Taylor-Couette measurements were additionally shifted from the onset shear rate observed in a benchmark comparison with a commercial rheometer using a cone and plate arrangement. Fardin et al. demonstrated the onset of shear banding (of a similar system) did have a dependency on the dimensions of the Taylor-Couette geometry but the authors of the work were less rigorous in controlling both radii and $\kappa$.

It can therefore be concluded that the ratio of curvature does play a role in the appearance of non-linear rheological behaviour of a shear-banding system, meaning that the phenomena is not solely dependent on the shear rate but additionally on how the deformation is applied geometrically. As changing the degree of inhomogeneity of the Taylor-Couette geometry does not appear to allow for the isolation of the material behaviour, it begs the question of how would a similar material system behave if the curvature was completely removed, in essence, in a geometry with true homogeneous deformation rather than approximated?

This thought experiment can be further probed by considering the link between the location of the high and low shear bands relative to the variation in applied stress. Shear banding in WLM systems has been comprehensively studied using a variety of rheo-visualisations techniques; NMR velocimetry, dynamic light scattering, ultrasound and particle tracking and they all show that the high shear rate band develops at the moving inner wall when studied in a Taylor-Couette geom-

\footnotetext{
${ }^{12}$ There needs to be sufficient sample present per imaging pixel volume (voxel) to get an adequate signal to resolve NMR information. Additionally there needs to be enough pixels across the width of the gap to be able to resolve the velocity profile shape. Minimum of 10 , however on the order of 25 is more ideal.
} 
etry $[47-51]^{13}$. If the curvature is removed from the geometry and the stress variation is now a constant (i.e. planar homogeneous flow) then which wall will the high shear rate band appear at, as both walls are then moving relative to one another and are indistinguishable? Will shear banding even eventuate? Or will a symmetric-like banding previously seen in a cone and plate geometry [52] be generated instead? To further study shear banding and potentially other non-linear rheological behaviour it is therefore desirable to have a shear geometry which imparts planar Couette flow for simple fluids.

\subsubsection{Planar Cylindrical Hybrid Design}

Genuine planar flow is mechanically complex to produce. The simplest condition would be generated between two infinite parallel plates producing planar-Couette flow as already shown in Figure 2.5. As stated, this is physically problematic to realise.

Outside rheology in fluid dynamics literature, alternative geometries can be found to generate planar Couette flow [53-55] to study turbulence. These comprise of two parallel sections of planar flow connected by two semicircular sections of circular flow to give a flow path the shape of a racetrack. This creates closed streamlines of sample flow. Designing geometries with closed streamlines is commonly done in liquid rheological studies, where it is done to eliminate end effects in one dimension and allow for an infinite maximum on the total amount of strain that can be applied to a sample. These features are necessary to increase usability of the novel geometry design too.

This formed the inspiration for a novel Planar Cylindrical Hybrid (PCH) shear geometry design for rheo-NMR, to study complex materials. The PCH design, construction, and preliminary testing featured as an aspect of the $\mathrm{PhD}$ thesis of Brox [4], assisted by summer research student Finnian Gray. The original iteration of the device is shown in Figure 2.15. This device utilised a racetrack shape (highlighted by Figure 2.15b) where the sample is trapped between a stationary outer wall and a band under tension between two rotors. This gave a fixed sample gap $1 \mathrm{~mm}$ (as mentioned in Sec 2.4.1) the whole way around. The shear is applied by rotating the band around two rotors like a conveyor belt. The device is designed to work with a Magritek style rheo-NMR accessory (driveshaft and motor) connecting with a similar T piece as shown for the TC geometry in Fig 2.14a and fits inside a $25 \mathrm{~mm}$ RF coil. The band is a silicone elastomer and the rest of the device is constructed from

\footnotetext{
${ }^{13}$ As they are so well documented in both rheology and rheo- techniques WLM solutions are considered model systems to illustrate non-linear mechanical responses.
} 


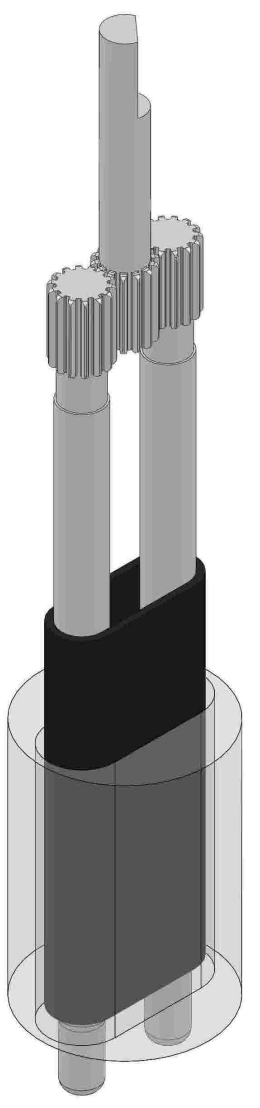

(a)

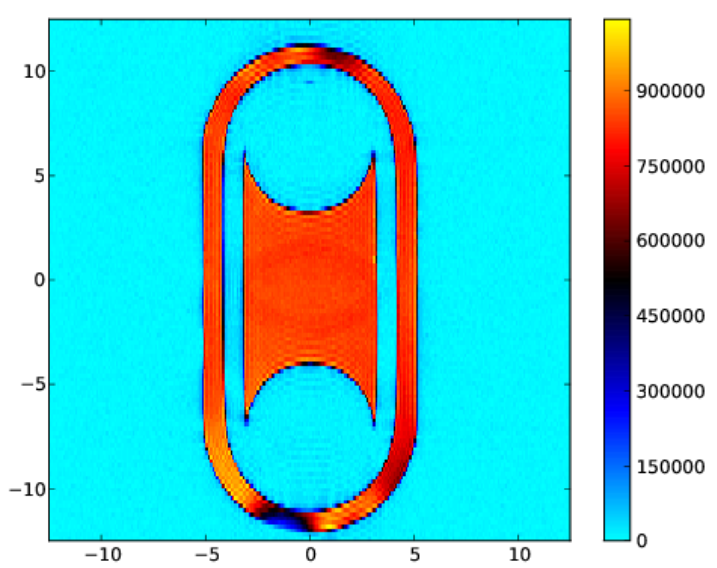

(b)

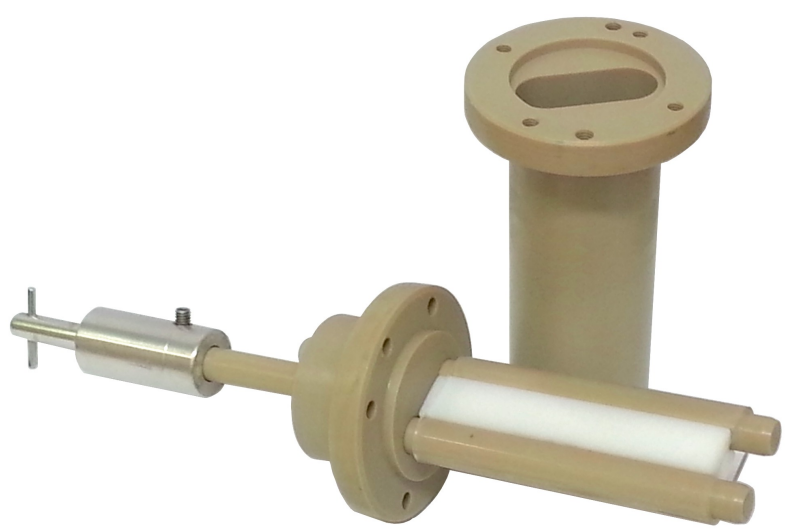

(c)

Figure 2.15: Description of original Planar Cylindrical Hybrid geometry design. (a) CAD cutaway showing the mechanics. The drive shaft couples to the top rotor pin whose teeth lock into two other rotors which act to turn the band, (b) MRI axial cross section of design where intensity of colour shows the sample region, the outer racetrack of sample is of interest to study (c) Photo of built geometry with inner wall component separated. Images used with permission of Tim Brox.

PEEK (polyether ether ketone) plastic, with the exception of a PTFE (ploytetrafluoroethylene) insert. These materials are important to the usability of the design as they are non-magnetic and mostly NMR inactive, meaning the sample material is solely responsible for the NMR signal detected. The rotors were $6 \mathrm{~mm}$ in diameter to be strong enough to hold the $1 \mathrm{~mm}$ thick band under tension. Optimising of these dimensions to achieve the greatest aspect ratio (length of planar region to gap 
thickness) possible is achieved. The outer walls fit a $23 \times 10 \mathrm{~mm}$ region, making it an order of magnitude smaller than previous devices used in fluid dynamics literature [53]. The PTFE insert shown in $2.15 \mathrm{c}$ was to support the planar sections of the band but was not used in testing.

Preliminary tests were done on the rheo-NMR PCH geometry with simple Newtonian fluids, water and PDMS (polydimethylsiloxane) oil (which has a higher viscosity than water) using a 2D PGSE-RARE NMR experiment to obtain velocity maps. These were compared to a fluid dynamics simulation run in with COMSOL Multiphysics which works by numerically solving the Navier-Stokes equation, see Figure 2.16.Looking across the planar gap with 1D slices for both methods were also compared. Qualitatively there was a good match between simulation and experiment and a linear velocity profile across the gap, however the maximum velocities were systematically less than those expected, and were thought at the time to be potentially due to wall slip.

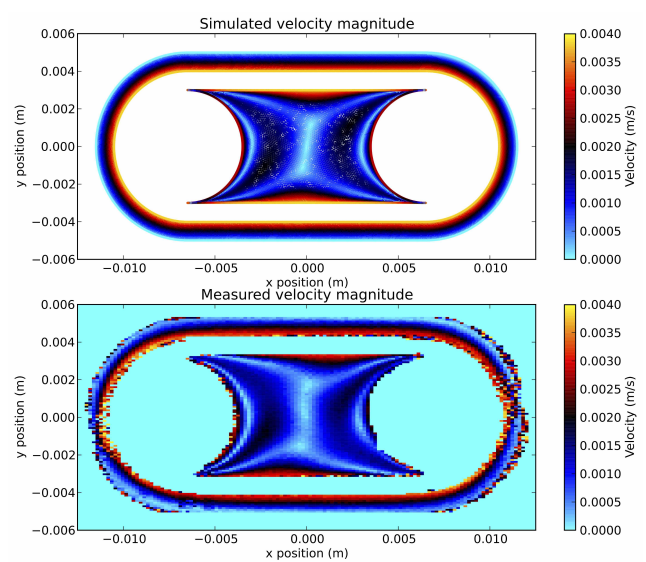

(a)

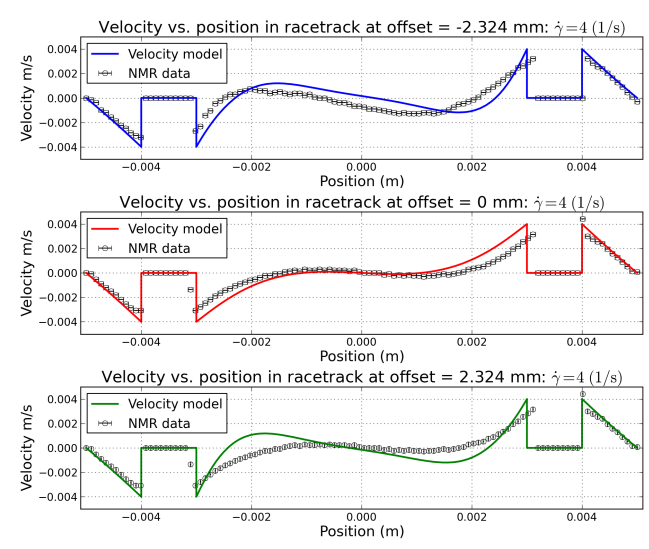

(b)

Figure 2.16: Results from preliminary testing of $\mathrm{PCH}$ with water at a shear rate of 4 /s. (a) A comparison of 2D COMSOL simulations of velocity magnitude (top) and NMR velocity map data (bottom), (b) a comparison of the NMR data with the COMSOL model at different positions across the racetrack. Used with permission of Finnian Gray.

The higher viscosity sample, PDMS oil, provided issues with loading and preventing leaking of the sample, and showed band distortion at higher shear rates, see Figure 2.17. 


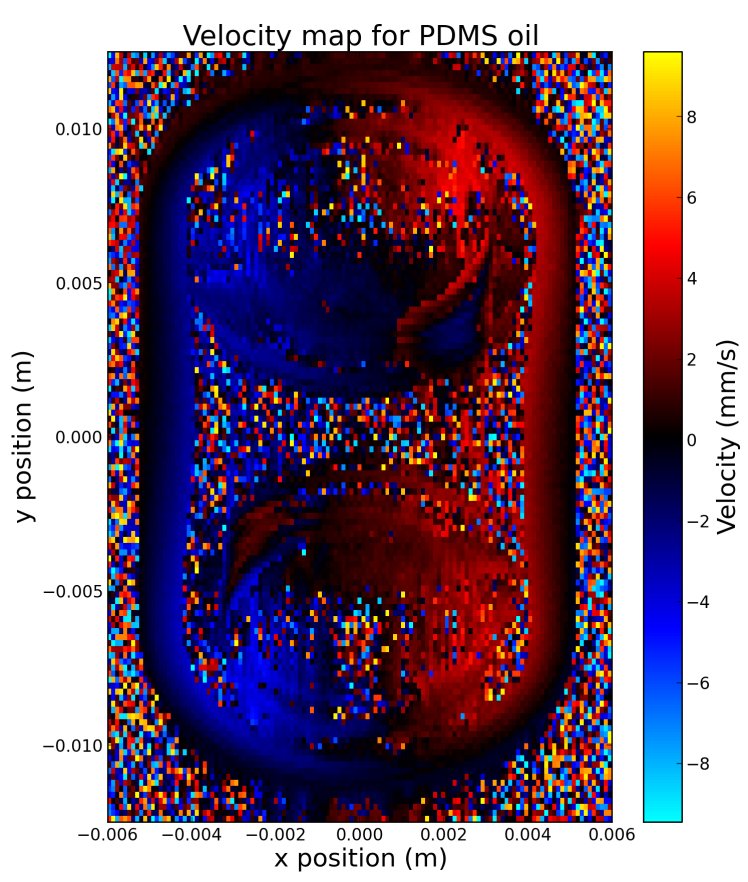

Figure 2.17: Velocity map showing a band distortion in planar regions for PDMS oil.

There was an issue with the constructed design in that the holes in the base of the geometry for the rotors holding the band to sit in were misaligned-aligned. This resulted in a twisting along the vertical direction. For testing this was managed by orientating the whole top such that the imaged region appeared properly aligned, however the potential secondary flows in the vertical direction were not accounted for.

\subsubsection{Further Work on PCH}

The Planar Cylindrical Hybrid (PCH) design and its preliminary results makes a compelling case to motivate further study, as it suggests simple Couette flow may be possible. What is required is updating the shear geometry design to address mechanical alignment issues in the first iteration and a refinement of testing methods is needed. Once done, an exploration can be conducted exploring the fluid dynamics and shear variation, as well as observing how a complex material (i.e. WLM) behaves. 


\section{Chapter 3}

\section{Experimental Methods}

The following chapter explores the necessary experimental methods that are later used to probe the second iteration of novel shear geometry introduced in Section 2.4. Included in this is the characterisation of the samples studied and a summary of the data acquisition and processing used to map the flow pattern in the geometry. This will be concluded with a section discussing anomalies in the NMR experiments and a record of considerations for future researchers using these techniques.

\subsection{Characterisation of Sample Fluids}

In the exploration of the novel shear geometry both a complex and a simple fluid are used. They are introduced below with focus on their relevant characteristics. This section begins with a brief comment on the rheometer preparation that is used for the complex material characterisation.

\subsubsection{Benchtop Rheometer}

As mentioned in Section 2.1.2, rheometers are instruments designed for the quantitative study of rheological properties by deforming / stressing samples and measuring their response. In this research a TA Instruments AR 2000 stress controlled rheometer (as shown in Figure 2.4) was used ${ }^{1}$. The set up and operation was as stated in manuals provided with the operation software [56, 57]. All measurements were made using a $60 \mathrm{~mm}$ diameter $2^{\circ}$ stainless steel cone and plate shear (drag flow) geometry. It offers an approximation to a homogeneous deformation with just $0.12 \%$ stress variation across sample gap.

\footnotetext{
${ }^{1}$ Note that the normal force balance was not operational and hence no normal force data will be presented.
} 
The rheometer environment was controlled to minimise potential sources of error. A custom built concrete slab table on rubber 'isolator' blocks to dampen building vibrations provided the benchtop surface for the rheometer. Rheological properties, in particular viscosity, can be heavily temperature dependant so effects were made to control sample temperature (at $25 \pm 2{ }^{\circ} \mathrm{C}$ ) with a Peltier element (underneath the plate of the shear geometry), a heat pump in the room and the door shut. Additionally all samples were left in the room for at least 30 mins prior to testing to equilibrate. As the WLM sample was light degrading, the room was lit solely by a desk lamp for these tests. The lamp created enough ambient light at the rheometer to ensure accurate loading and to monitor the sample during testing (as at high shear rates the sample had a tendency to be ejected). The electronics control box housing for the rheometer shaded the sample vessel and geometry from direct light.

\section{Instrument Checks}

While an operator of the rheometer is unable to manually calibrate it, checks were done, as per manuals, to ensure that it was operating as expected. Sources of error highlighted were:

1. System calibration factors (i.e. geometry height and inertia of the system were required to be reset each time the geometry was reconnected),

2. Peltier plate temperature,

3. Sample loading (over or under-filling the geometry effectively changes the diameter and may introduce more prominent edge effects).

The first was incorporated into operating procedures and the latter two were verified.

The temperature settings of the Peltier element were tested with a K-type digital thermocouple in thermal contact with the geometry plate. Thermal paste (RS Heat Sink Compound Plus) was used to improve contact and a tea towel placed over top to better simulate sample trapped between the two geometry surfaces (with little surface area in direct contact with the room). The Peltier element was set from $0.0-50.0^{\circ} \mathrm{C}$ in steps of $10.0^{\circ} \mathrm{C}$, after the feedback from the Peltier had been stable for over a minute the temperature was recorded with the thermocouple. Results are displayed in Figure 3.1. The slight variation in the readings was insignificant compared to the thermocouple error $\pm 1^{\circ} \mathrm{C}$ and the overall comparison was deemed in good agreement. 
Fill amount effects were explored by comparing flow curves from a batch of the complex (shear banding) sample for a range fill amounts encompassing a difference of $\pm 5 \mathrm{~mm}$ of the geometry radius $(30 \mathrm{~mm})$, Figure 3.2. It is clear the significant effect that under-filling can have on the processed data. Slightly over and under fills (deemed to be the boundary of the acceptably repeatable range) gave variation in shear stress of approximately $\pm 8 \%$.

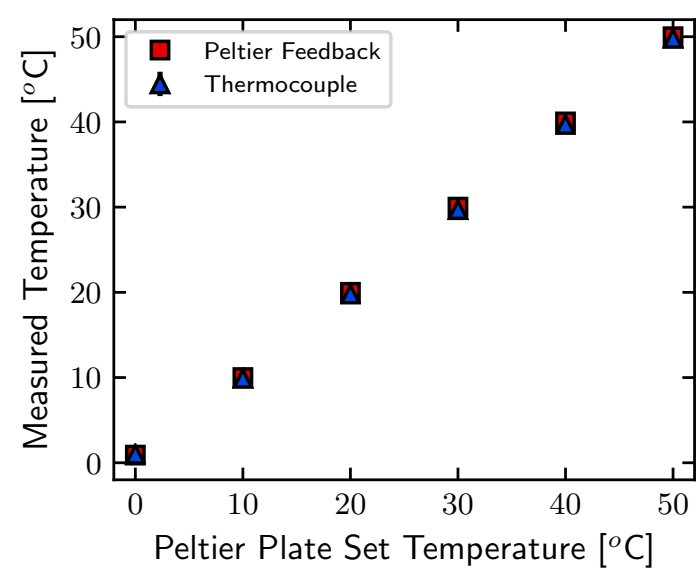

Figure 3.1: Temperature calibration test for AR 2000 rheometer. (red) Temperature obtained by the rheometer according to its feedback reading, (blue) are the $\mathrm{K}$ type thermocouple output. Fluctuations in reading were smaller than the error associated with thermocouple accuracy, which is too small to be visible on the plot.

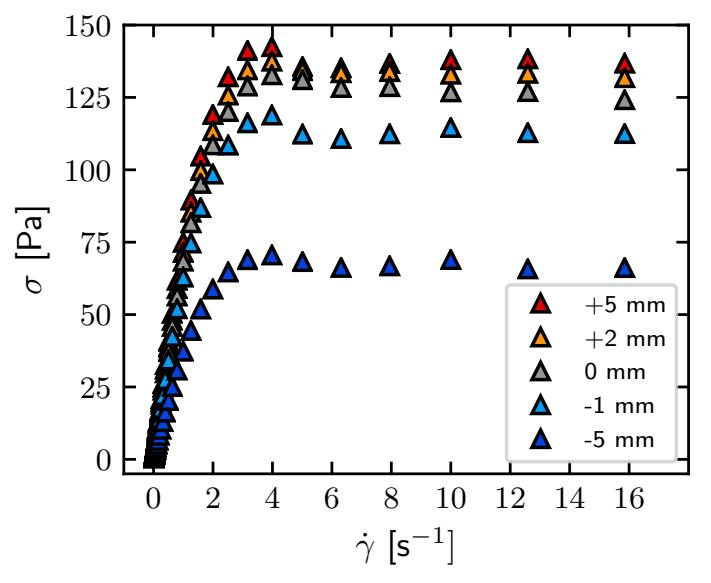

Figure 3.2: Fill amounts testing using a shear banding sample. The labels indicate the change to the nominal geometry radius $30 \mathrm{~mm}$ and are to the nearest $\mathrm{mm}$. 
The viscosity output values were reinforced by tests using three silicone oils of differing viscosities. From Table 3.1 the percentage difference can be calculated and the measured values are less than the maximum $4 \%$ variation which is acceptable [56].

\begin{tabular}{c||c|c} 
Sigma-Aldrich PN & Stated Viscosity [Pa s] & Measured Viscosity [Pa s] \\
\hline \hline 378402 & 9.71 & 10.00 \\
181838 & 58.8 & 57.39 \\
378437 & 97.1 & 100.35
\end{tabular}

Table 3.1: Viscosity data for the three silicone oils measured by the TA Instruments rheometer compared to the stated nominal viscosities of the product. Note these oils are not viscosity standards and so do not have an associated error. The variation in raw measured data was less than $1 \%$.

\section{Types of Experiments and Uses}

There are four types of rheometer experiments that are applicable to this research, two steady flow and two oscillatory experiments.

A 'peak hold' flow experiment holds the shear constant and records stress data at defined intervals in time. The resulting plot of the non-held shear value (either stress or shear rate) reveals any start up flow which can be used to determine waiting times for a sample to settle into a flow regime.

A 'stepped flow' flow experiment entails applying successive shear values in time steps, where data is collected at the end of each step leaving the sample time (determined by a peak hold experiment) to settle into the flow regime dictated by the shear rate. This produces a flow curve.

Oscillatory tests include a 'frequency sweep' and 'amplitude (strain) sweep'. The frequency sweep experiment is used to obtain plots for the loss and storage moduli $\left(G^{\prime \prime}\right.$ and $\left.G^{\prime}\right)$ to study the viscoelasticity of the sample. A strain sweep oscillates at a set frequency and increments the strain. The resulting plot is able to show the separation of the linear and non-linear mechanical response regions for deformations. From the linear response region a strain value is selected to hold constant for a frequency sweep test.

The mathematical theory of viscoelastic materials includes a subfield concerning linear viscoelasticity (linear relationship between stress and strain, so the response at any time is directly proportional to the initial stimuli). In this the dif- 
ferential equations of time derivatives for stress and strain are linear. There exists a variety of models to explain viscoelastic responses as a sum of time derivatives, differing by which time derivative they deem to be non-zero. The simplest cases are with the zeroth and first time derivatives of strain relating to stress. These yield equations (2.1) and (2.2) respectively. A more complex model is the Maxwell model $\sigma+\tau \dot{\sigma}=\eta \dot{\gamma}$, where $\tau$ is the relaxation time describing the competing visco and elastic responses ${ }^{2}$. Relating this model back to the complex relaxation modulus equation (2.5) it can be shown that for oscillatory shear $G^{\prime}$ and $G^{\prime \prime}$ are functions of frequency

$$
\begin{aligned}
G^{\prime} & =G_{0} \frac{\omega^{2} \tau^{2}}{1+\omega^{2} \tau^{2}} \\
G^{\prime \prime} & =G_{0} \frac{\omega \tau}{1+\omega^{2} \tau^{2}}
\end{aligned}
$$

with $G_{0}=\eta / \tau$ the relaxation modulus amplitude. $\tau$ is also referred to as the characteristic natural time for the response ${ }^{3}$.

\subsubsection{Doped Water}

One sample used in the thesis is a simple Newtonian fluid. This was done to trial the experimental methodology on a standard sample and to explore the fluid dynamics of the novel geometry compared to a TC geometry.

Distilled water doped with $0.15 \mathrm{mM} \mathrm{GdCl}_{3}$ was used for this. The $\mathrm{GdCl}_{3}$ was added to reduce the $T_{1}$ NMR relaxation time to allow for a reduction in time between successive NMR scans. Two batches of doped water were made starting from pre-diluted samples in the general lab stock. For these the relaxation times were $T_{1} \approx 570 \mathrm{~ms}$ and $T_{2} \approx 510 \mathrm{~ms}$ for batch one and $T_{1} \approx 440 \mathrm{~ms}$ and $T_{2} \approx 400 \mathrm{~ms}$ for batch two.

\subsubsection{Wormlike Micelle Surfactant System}

As mentioned earlier, wormlike micelle (WLM) solutions are an example of a well documented shear banding material (pages 16 - 17). In terms of soft matter they are surfactant solutions, classified as amphiphilic, with a solvent loving head and a solvent hating tail (often a long carbon chain). Depending on their chemical environment this property results in them self-organising into complicated structures. In

\footnotetext{
${ }^{2}$ This is a different $\tau$ from the one used to name certain delay times in an NMR experiment.

${ }^{3}$ See Barnes et al. [8, Chapter 3] for derivations.
} 
WLM solutions these are elongated flexible and cylindrical, hence they are named after their animal similarity.

The WLM solution chosen to help explore the PCH geometry in this thesis was similar to that used in both the development of PGSE-RARE pulse sequence employed in this thesis, Figure 2.11, and in the paper looking into the influence of curvature in TC geometries which was part of the novel geometries motivation (see $[3,31]$ respectively). It is made of $8.09 \mathrm{wt} . \%(0.238 \mathrm{M})$ cetypyridinium chloride $(\mathrm{CPCl})$ and $1.9 \mathrm{wt} . \%(0.119 \mathrm{M})$ sodium salicylate $(\mathrm{NaSal})$ diluted in a distilled water brine $\left(0.5 \mathrm{M} \mathrm{NaCl} \text { and } 0.15 \mathrm{mM} \mathrm{GdCl}_{3}\right)^{4}$. The total $10 \%$ weight concentration regime of $\mathrm{CPCl}$ and $\mathrm{NaSal}$ makes this a 'semi-dilute' solution where the system is viscoelastic.

The preparation of the samples involved weighing out the desired quantity of the NaSal salt into a $50 \mathrm{~mL}$ volumetric flask and dissolving with the $0.15 \mathrm{mM}$ $\mathrm{GdCl}_{3}$ brine. This was repeated for the two chloride compounds $\left(\mathrm{NaCl}\right.$ and $\left.\mathrm{CPCl}^{5}\right)$ into a $200 \mathrm{~mL}$ volumetric flask, however the mixture required heating (between $30-50^{\circ} \mathrm{C}$ ) and swirling to remove bubbles before it could be cooled back down to room temperature and topped up to the correct volume. Stirrers were added to each flask and they were alternatively mixed on a hot plate for 30 mins each $(2 \times$ 15 min sessions) before combining the final solution into an amber jar. The jar was wrapped in aluminium foil to protect from potential light degrading. To ensure proper development of the WLMs, the jar was placed on rollers overnight, then left for several days in a drying cabinet set to $30^{\circ} \mathrm{C}$. Three batches were made in total.

The rheometer from Section 3.1.1 was used to characterise the bulk rheology of the WLM batches before NMR experiments were performed. For obtaining flow curves a stepped flow test was used, logarithmically stepping the shear rate from $0.01-100 / \mathrm{s}$ (though occasionally restricted up to $20 / \mathrm{s}$ as sample was ejected from geometry after that) with 10 points per decade. A sampling time of $5-10 / \mathrm{s}$ was used in conjunction with a maximum equilibrium prior wait time of $60 \mathrm{~s}$ (discussed later in this section). Results are shown in Figure 3.3.

From this the shear measurements can be seen to plateau to approximately $120 \mathrm{~Pa}$ after a shear rate of $\approx 3 / \mathrm{s}$. The difference among the samples is small and within the $\pm 8 \%$ error that comes from the accepted fill amount variation.

The shear storage $\left(G^{\prime}\right)$ and shear loss $\left(G^{\prime \prime}\right)$ moduli were measured using a frequency sweep oscillatory experiment, taken a strain of $4 \%^{6}$. The frequency of

\footnotetext{
${ }^{4}$ All chemicals were sourced from Sigma-Aldrich except for the $\mathrm{NaCl}$ which was from Pure Science.

${ }^{5} \mathrm{CPCl}$ was a monohyrdate and this water mass was accounted for.

${ }^{6}$ Determined by a strain sweep experiment to be well in the linear response regime.
} 


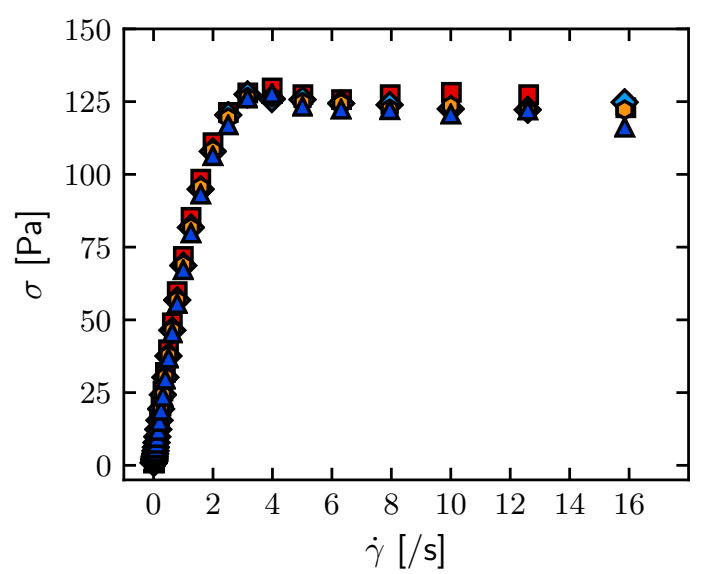

Figure 3.3: Flow curve for the three batches (squares, diamonds, triangles) of WLM solution measured before NMR experiments. (hexagons) The flow curve of batch two taken after the end of NMR experiments to see if sample degrades while in amber jar.

the oscillations was logarithmically stepped from $0.01-100 \mathrm{~Hz}$ with 10 points per decade, see Figure 3.4. Dashed lines show the fit of the Maxwell model with $G_{0}=190 \mathrm{~Pa}$ and $\tau=0.39 \mathrm{~s}$ within the expected range for this WLM sample.

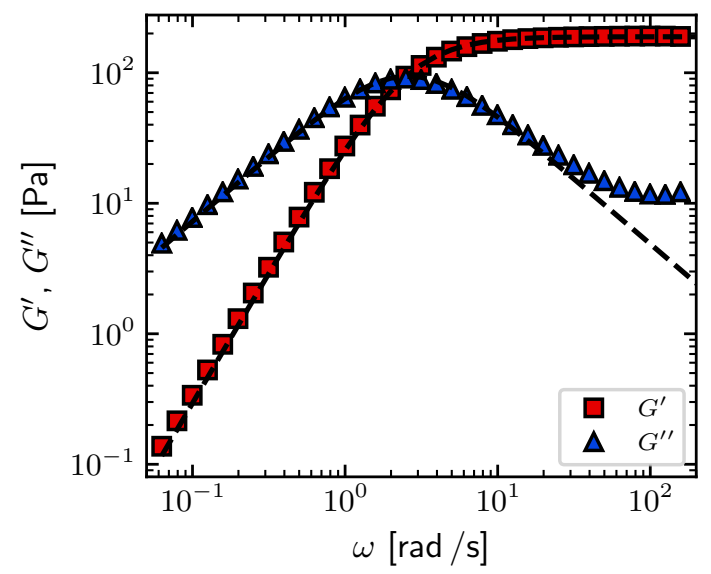

Figure 3.4: Frequency sweep data on the second batch of WLM solution at a $4 \%$ strain. Dashed lines are the Maxwell model fit for equations (3.1) and (3.2).

A peak hold experiment provided the start up dynamics of the WLM solutions, Figure 3.5. The shear rate was held at three different values $(\dot{\gamma}=0.5,5,10 / \mathrm{s})$ and the resulting stress was measured as a function of time. The two shear rates above 
the onset of shear banding show a trend similar to that found by López-Barrón et al. [58] with a stress overshoot then a multi-step decay down to a stress plateau. The overshoot and start of stress plateau were seen at $t \approx \tau$ and $t \approx 10 \tau$ respectively in Lopez-Barron et al., however the shift in the timing that we see here is believed to be due to stress controlled nature of the rheometer that means it undergoes a sinusoidal feedback process to achieve a constant shear rate. The rheo-NMR drive shaft is strain controlled and so the current stress controlled case will be the limiting factor for start up.

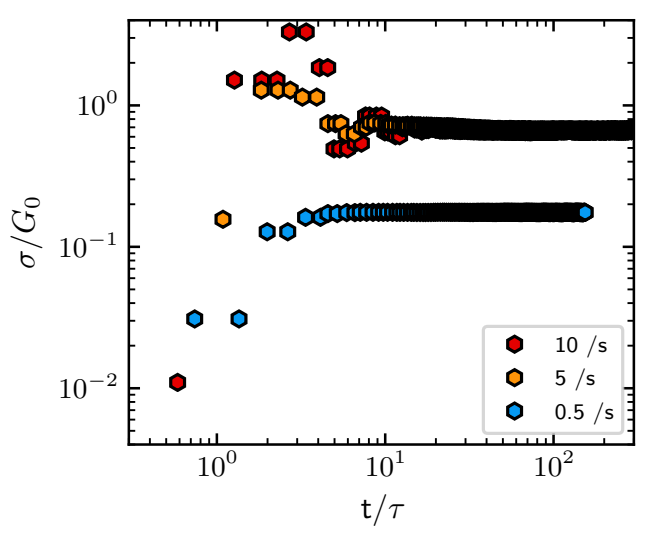

(a)

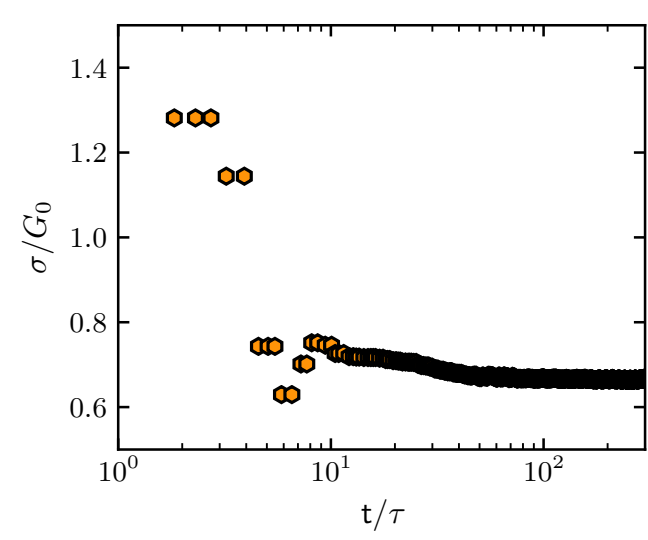

(b)

Figure 3.5: (a) Start up flow experiment at $\dot{\gamma}=0.5 / \mathrm{s} \mathrm{(blue),} \dot{\gamma}=5 / \mathrm{s}$ (orange) $\dot{\gamma}=10$ /s (red) with both stress and time as a ratio of viscoelastic parameters. (b) Zoomed in plot of $\dot{\gamma}=5 / \mathrm{s}$ time evolution, which shows additional decay after $t \approx 10 \tau$ with a longer relaxation time.

The rheological behaviour of the WLM solution was taken into consideration for the timing of the NMR experiments. Considering the Maxwell model relaxation time and the time-dependent steady shear experiment, it was decided that a $1 \mathrm{~min}$ pre-shearing would be allowed to occur before any NMR data would be collected. The NMR experiments themselves were approximately 3 mins for 8 repeat scans, after which the shear was stopped and a minimum of 5 mins was allowed to elapse before the other shear rate could be performed.

Similar to the water sample, $\mathrm{GdCl}_{3}$ is used to reduce $T_{1}$ for the WLM solution. Of the three batches made, batch one came from the first stock of doped water and had a final $T_{1}$ and $T_{2}$ of $\approx 400 \mathrm{~ms}$ and $\approx 200 \mathrm{~ms}$. The latter two were made of the second batch of doped water and had a final $T_{1}$ and $T_{2}$ 's of $\approx 450 \mathrm{~ms}$ and $\approx 220 \mathrm{~ms}$. This same solution was made by Feindel and Callaghan [59] with and without the 
$T_{1}$ reducing agent showing that its inclusion had negligible change on the rheology of the overall solution.

\subsection{Data Acquisition and Processing}

To explore the flow fields of the two aforementioned samples inside the novel $\mathrm{PCH}$ and the benchmark TC shear geometries, the NMR velocimetry experiments described in Section 2.2.4 (in particular, pages 25 to 26) were employed. In this section different sampling orientations for those two methods are discussed, including how they are processed to yield the plots and maps in Chapter 5.

\subsubsection{Flow Volumes Sampled for 2D and 1D Plots}

Earlier it was introduced that NMR imaging (or simply spatial encoding) is done through the use of three orthogonal gradient coils. These coils define a laboratory Cartesian frame $x, y, z$, where $z$ is, by convention, along the axes of the $B_{0}$ field (vertical in this case). For imaging there are three orthogonal axes read, phase, slice $(r, p, s)$ shown in Figure 3.6. The laboratory axes can be assigned to the imaging axes in any order either exactly or under a rotation. Meaning that 3D space can be explored from every possible viewpoint. For this work there is a particular interest in encoding for velocity perpendicular to a symmetry plane in the shear geometries and hence all experiments were either manually aligned with the laboratory frame or artificially rotated using a rotation matrix to assign $r, p, s$ to $x, y, z$.

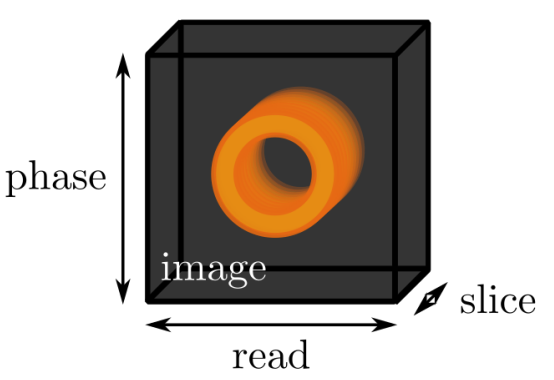

Figure 3.6: MRI dimension labels.

Using the symmetry of the two shear geometries, three slice orientations are defined for PCH and two for TC. These are displayed in Figure 3.7 where the image's surface is shown for the axial slice (thickness of image is along $z$ ) while only the 
thickness of the other orthogonal slices (as they are viewed by an observer looking along the surface of the page) can be seen.

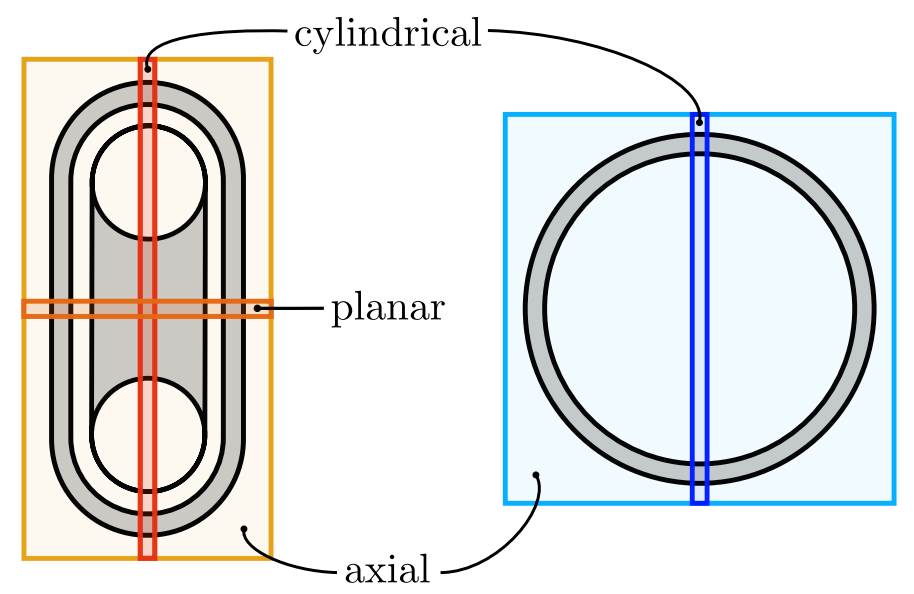

Figure 3.7: Names and corresponding orientations for different slices for both the novel PCH and TC shear geometries that will be referred to in this thesis. Slices are respectively orthogonal.

The 'cylindrical' slices look at the curved regions of the geometries (for TC this is independent of rotation in the plane of the page), while the 'planar' slice looks along straight region of the $\mathrm{PCH}$ that is ideally inducing the desired planar-Couette flow. Flow can be encoded along the height, width, or thickness of each slice orientation. The thickness of these slices (the volume depth over which the signal is averaged for the image) was typically $1 \mathrm{~mm}$ to balance the interplay between losing smaller scale velocity information, and having a voxel (3D imaging pixel volume) that includes sufficient sample to give satisfactory signal-to-noise.

The length of read and phase image dimensions (see Figure 3.6) are labelled by a Field Of View $(\mathrm{FOV})^{7}$. The number of pixels chosen for each direction determine the overall image resolution. For a fixed FOV, more pixels translate to small voxels (weaker signal-to-noise). The resolution was selected to obtain $\approx 16-26$ pixels across the sample gap of $1 \mathrm{~mm}^{8}$ in the geometries to resolve the velocity profile and still have sufficient signal.

\section{D velocity maps}

The PGSE-RARE pulse sequence was used obtain 2D velocity maps of the $\mathrm{PCH}$ and TC geometries to observe the evolution of flow around the sample region. Figure

\footnotetext{
${ }^{7}$ i.e. $20 \times 20 \mathrm{~mm}^{2}$ produces a square image with $20 \mathrm{~mm}$ sides.

${ }^{8}$ Discussed in Section 2.4.1.
} 
3.8 shows an example, with the velocity maps on the left. The colour (blue / red) indicates the direction of flow, and the lighter / brighter the colour the faster the velocity (i.e. black regions represent stationary or average stationary flow). As such, the colour is brighter closer to the inner wall of each design as they are the moving surfaces.

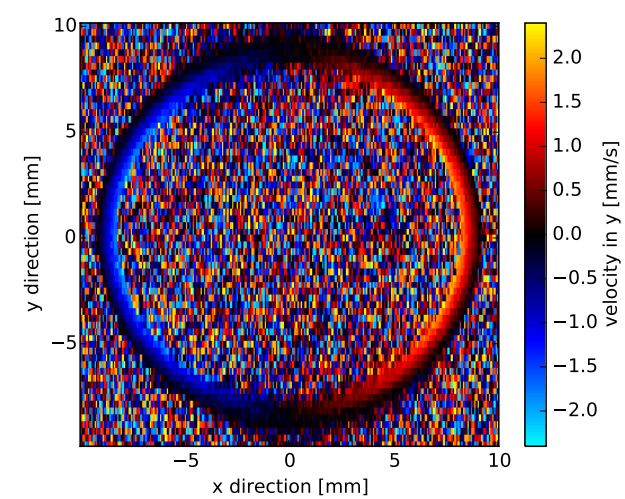

(a)

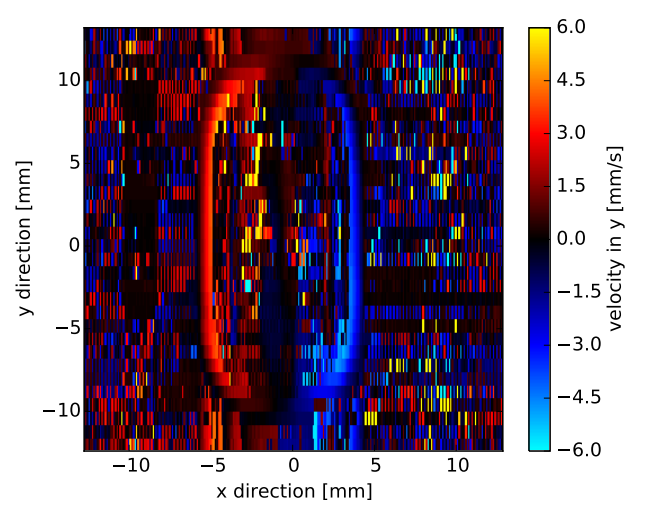

(c)

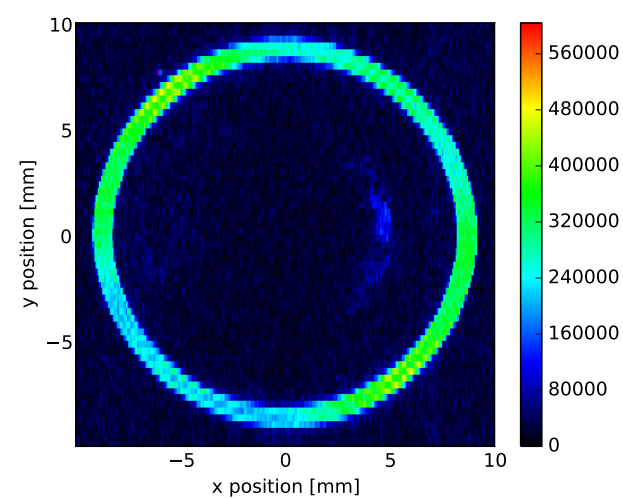

(b)

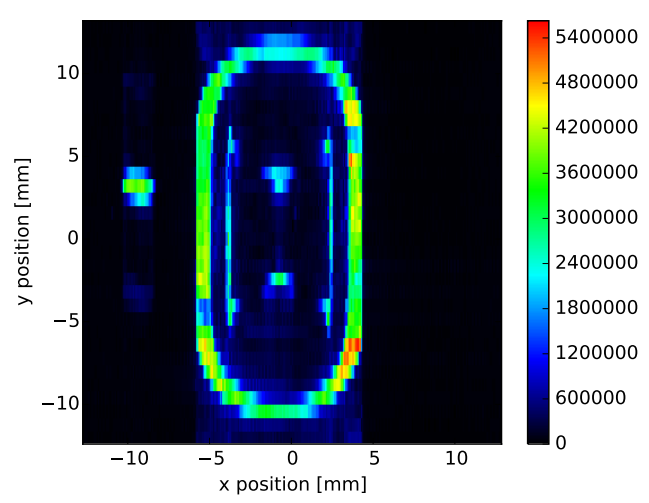

(d)

Figure 3.8: Examples of PGSE-RARE 2D maps: (top row) TC shear geometry and (bottom row) $\mathrm{PCH}$. The left column shows the velocity maps with velocity encoded in the height direction of each image. On the right is the corresponding axial RARE images of the geometries.

To study a planar region of the novel shear geometry, it can be useful to isolate a single planar section in the velocity map. The image of the entire geometry, along with the velocity map, helps determine the pixels that represent sample in the planar region so that the velocity map can be cropped to just include these pixels. An example of a crop is shown in Figure 3.9. 


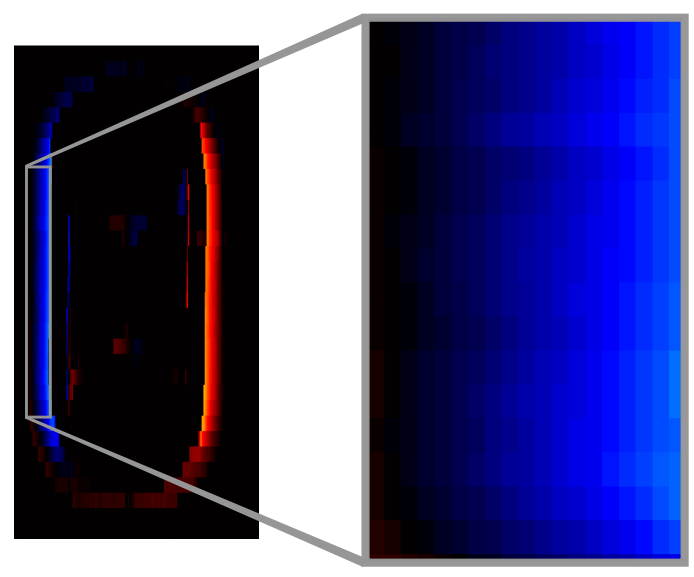

Figure 3.9: Selection of planar region from 2D axial velocity map.

\section{$2 \mathrm{D}$ velocity maps to $1 \mathrm{D}$ velocity profiles}

To probe the flow behaviour over a range of shear rates, the same pulse sequence was used to take 2D velocity maps for $1 \mathrm{~mm}$ thick planar and cylindrical slices. This produces a velocity map like that in Figure 3.10a, with the second imaging direction (phase) along $z$. Each column in the map is averaged to produce a single value and the string of all columns gives a 1D velocity profile, Figure 3.10b. Much more for the $1 \mathrm{D}$ plots than in the $2 \mathrm{D}$ velocity maps, the shape and magnitude of the velocity profile from the inner moving wall to the outer stationary wall is evident. Figure $3.10 \mathrm{~b}$ shows the expected linear profile for the Newtonian fluid (water). From repeating this processing method for tests at a range of different shear rates, then super-imposing the respective sample gaps on top of one another (see thresholding section for gap picking discussion) can a comparison be made on how the shape and magnitude of the velocity profile evolves as a function of shear rates.

Note that in the refinement of the PGSE-RARE pulse sequence by Kuczera [60] there is the discussion of the observed variation in the $z$ (vorticity) direction with a WLM sample that has also be seen in other rheo-visualisation techniques [61, 62]. For NMR visualisation Kuczera showed that the periodicity of the undulations in the vorticity direction is on the order of the chosen pixel size $\approx 1.25 \mathrm{~mm}$ and hence not resolved. Variation in $z$ was not significant in tests performed for this work either. Averaging over the $z$ was therefore able to be utilised to improve results.

\section{D velocity profiles}

$1 \mathrm{D}$ velocity profiles were also acquired directly using the 1D double slice selection pulse sequence. A second slice selection occurs in the phase direction, meaning 


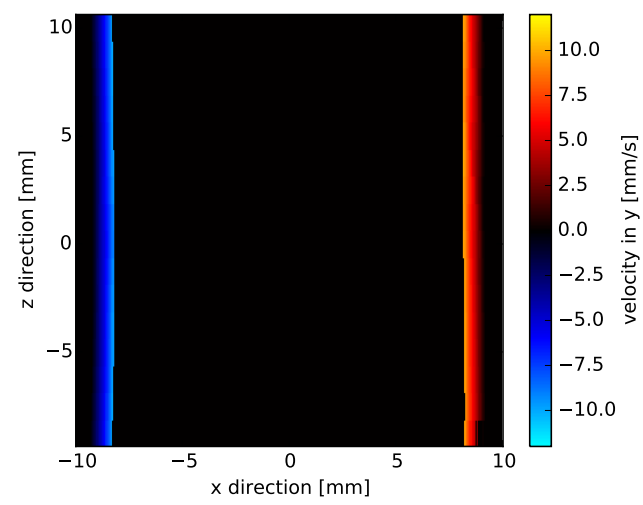

(a)

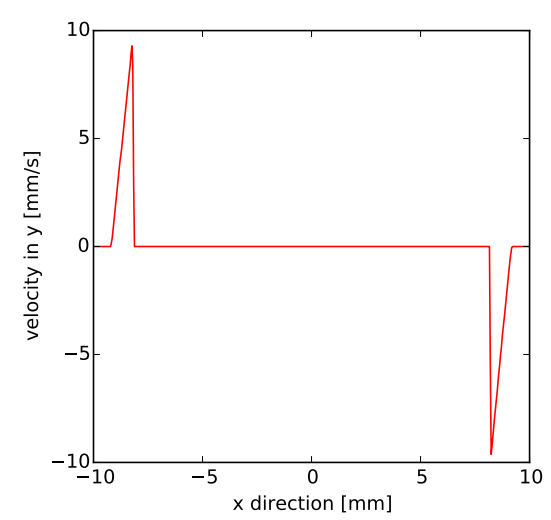

(b)

Figure 3.10: (a) 2D cylindrical slice velocity map for TC geometry, with 16 steps in the vertical direction over $20 \mathrm{~mm}$ length. (b) 1D velocity map obtained from averaging over center $10 \mathrm{~mm}$ along $z$. Data outside the center $10 \mathrm{~mm}$ is not averaged over as field inhomogeneities start to weaken the signal.

the phase FOV is sampled by a single slice. Read is the only spatially resolved dimension. This effectively does the collapsing down of the signal along $z$ that was done manually to get Figure 3.10b. However the velocity information can also be displayed in a 2D format such as Figure 3.11. The maximum intensity, in this 2D map, is the mean velocity sampled as function of the physical read dimension.

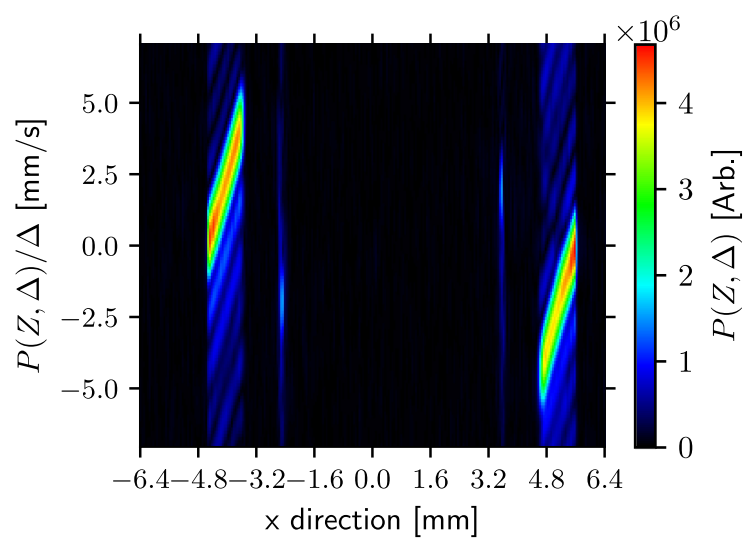

Figure 3.11: Map showing the propagator spatially resolved. Here the horizontal axis represents a real dimension, i.e. $x$, while the vertical axis is the corresponding highly under-sampled and zero-filled propagator converted into velocity. The intensity scale is the magnitude of the propagator in arbitrary units. The mean velocity is still contained by the peak of the propagator function. 


\subsubsection{Data Processing and Thresholding}

To produce the images, velocity maps and velocity profiles described above the raw NMR data requires processing.

MRI experiments run with ParaVision (usually to check set up conditions including orientation) have outputs displayed as an already processed image. However the majority of testing for this thesis was conducted through TopSpin software where, except for $T_{1}$ analysis, data is exported and processed separately. Magtriek's Prospa analysis software helped process $T_{2}$ data and 2D images / velocity maps. For further user controllable processing, Python scripts were used. These scripts were adapted from those used by past and present members of the Magnetic Resonance Physics group at Victoria University of Wellington, Stefan Kuczera and Tim Brox. The python scripts processed both 1D and 2D image / velocity data and extracted 1D information from 2D experiments.

The basis for data processing lies in Fourier (and inverse Fourier) transforms. Fourier transforms translate imaging dimension data from k-space to position (real) space. Moreover they can be used for velocity encoded data processing. In this technique for generating velocity data, Fourier transforms turns the collected qspace data to a form of displacement space. This generates the columns of Figure 3.11 , of which the position of peak value $R_{p}$ represents the velocity $v$ for a known displacement time $\Delta$ through equations

PGSE-RARE

$$
v=\frac{1000}{\gamma \delta \Delta g_{\max }} \frac{2 \pi}{Z} \frac{q_{\text {dim }}}{2}\left(R_{p}-\frac{Z}{2}\right)
$$

1D double slice selection

$$
v=\frac{1000}{\gamma \delta \Delta g_{\max }} \frac{2 \pi}{Z}\left(q_{\text {dim }}-1\right)\left(R_{p}-\frac{Z}{2}\right)
$$

where $q_{d i m}$ is the number of different $q$ (gradient) steps that were used in the NMR experiment, $g_{\max }$ is the maximum gradient strength used to collect the q-space data, and $Z$ is the zerofill used to smooth q-space data. The two forms of the equations come from how each pulse sequence 'steps' or transverses through q-space. The 1D velocimetry experiment steps linearly from zero to positive $\left(q_{d i m}-1\right)$ times the $q$ step size (restricted by the phase winding gradient increments $<2 \pi$ for certain Fields Of Flow (FOF) or expected velocity ranges). The PGSE-RARE used here steps linearly from negative to positive q-space, still with the same number of steps one of which is zero, hence the maximum comes from $q_{d i m} / 2$ times the $\mathrm{q}$ step size.

Velocity maps and profiles are thresholded to lift the actual sample velocity data from the surrounding noise. This is implemented from the Python scripts by using 
the RARE image (in the PGSE-RARE case) that is generated alongside the velocimetry map to create a mask. The mask takes all the pixels in the image that are above a certain chosen threshold factor (i.e. 0.5) times the maximum signal value in the image and creates an equivalent matrix with corresponding entries labelled 1 , and 0 for regions where the pixel signal is below the threshold. Multiplying this mask by the velocity map produces a plot where the only regions with velocity information are those which actually have a detectable sample above a certain threshold to begin with.

To make a quantitatively comparison of velocity profiles for different NMR experiments, it is essential to know where the sample gap is in the processed data. This will determine a fixed point to plot all data in reference to. Due to potential mechanical wobble or movement of the inner wall of both geometries the outer wall was used as this reference point. It can be established from the masked data when the signal goes from zero to non-zero. As pixels have a finite length it is not a significant concern that the first true sample pixel by the outer wall would be exactly zero. Using this method and just extracting the velocity information across the gap can give plots like Figure 3.12.

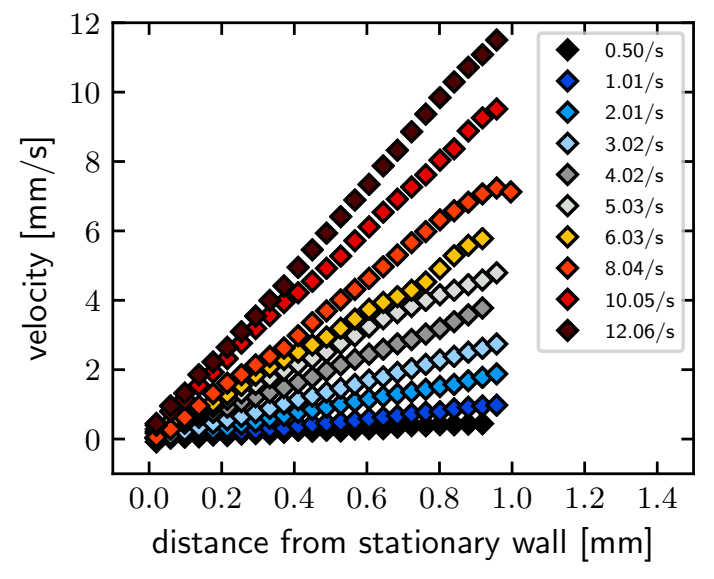

Figure 3.12: Example of $1 \mathrm{D}$ velocity data extracted over a single sample gap for a range of shear rates.

\subsubsection{Pixel Location}

Discussed earlier, in terms of pixel resolution, was the idea of the trade off between sufficient signal-to-noise and enough data points to clearly map the velocity distribution of a sample under shear. For narrow high shear rate bands in a WLM sample 
or cases of wall slip, a high resolution is desirable. These close-to-the-wall features may be further obscured by the location of the voxel in space. The pixels have a finite width (typically around $40-50 \mu \mathrm{m}$ ) and could overlap both undetectable wall material and sample. For images, this simply results in a weakened intensity proportional to what percentage of the voxel is wall, assuming even density of spins in the sample. In velocity encoding, a voxel's velocity is the average velocity of all the sample present in it. A $50-50 \%$ pixel at the moving wall will have an average velocity lower than that of the moving wall yet its marked location in space is at the wall location. This shifts the detected velocity down at the moving wall and up on the stationary wall. Consequently a $\pm 1 / 2$ pixel width error should be considered for all images. As the stationary wall is used as a reference point, all plots in the form of Figure 3.12 will have the first point inset half a pixel width, which factors in the error and thresholding methods.

\subsection{NMR Velocimetry Considerations}

The NMR velocimetry pulse sequences have been used for previous studies, and the PGSE-RARE sequence explored in detail by Kucera [31, 60]. To gain familiarity as part of this research additional validation studies were conducted. Through this process it was noted the variety of influences that can effect the final NMR results. A few of these can be mitigated, while others are considerations that an NMR practitioner should be aware of.

\subsubsection{Mechanical Wobble}

Mechanical wobble (or misalignment) is a concern with any kinetic design. In this specific case it could impact the flow conditions that the samples are subjected to, so a potential issue to be aware of. Improvements in both the rheo-NMR TC geometry (made prior to this study) and the $\mathrm{PCH}$ geometry (discussed in Chapter 4) aimed to minimise mechanical wobble. The residual effect of wobble can be monitored in some NMR experiments as an increased gap width in the image, doubling of maximum velocity line in 2D propagator plots, or physical shifts in the gap width when plotting an image with a q dimension. In the $\mathrm{PCH}$ geometry, the band position relative to the outer stationary wall may vary by $\approx 150 \mu \mathrm{m}$, not unsurprising considering the number of influences on its behaviour (mold accuracy, placement of rotor under tension, dynamics of fluid on inside edge of band etc.). In data at increased band speeds the wobble is less apparent. When data was collected for a range of shear rates (approximately an order of magnitude difference) the displace- 
ments measured were constant and instead $\Delta$ was varied to compensate. Using 8 repeat scans for each q-step (improve signal to noise) and a repetition time of $\approx 2.5 \mathrm{~s}$ (governed by $T_{1}$ ) resulted in at least $20 \mathrm{~s}$ time elapsing per q-step. For shear rates an order of magnitude apart that translated to sampling a portion of the band versus several rotations. Consequently mechanical wobble may still be present at higher shear rates, however it is lost to the observer potentially due to averaging effects over a single q-step.

\subsubsection{NMR Repeatability}

Repeatability of an experiment under identical conditions is key when the focus is on detecting potentially subtle differences in sample flow. Specific tests were run immediately after, a day after, or several months after (with same style geometry and new sample) for the PGSE-RARE with good agreement, which builds confidence in the technique. Observed differences were usually due to mislabelled gradients or odd q-step influences (commented on below), both can be managed to an extent.

Results presented later in the thesis took the approach of mechanically reversing the flow direction. The flow environment does not have any irreversible features and logically reversing the flow direction should not yield any differing results for the evolution of the velocity profile along the sample track. This was another way to confirm results observed were repeatable.

\subsubsection{Unwanted Magnetisation in a Single q-step}

A further consideration with position encoded velocimetry experiments is the refocusing of undesired signal. The unprocessed data for velocimetry experiments is displayed in the time domain on completion of an experiment. It is segmented by 8 q-steps. It was not unusual for one of these q-steps to have a signal visibly larger than the rest. The processed data for that q-step produces a distorted image which may show through in odd sections of flow in the velocity data. The issue comes from having both slice selective gradients and q-encoding gradients acting on the same direction. Slice selection utilises a soft $\pi$ pulse during an applied gradient from which only the frequencies inside the bandwidth of the pulse are exactly dephased and rephased by the first and second halves of the pulse. Everything else loses coherence. However through specific cases of timings and strengths of PGSE gradients, a dephasing is imparted on the spins, of which some of this magnetisation that would otherwise be lost is being rephased by the slice selection gradients. 
A similar approach appears in spectroscopy, coherence pathway selection, where gradients and pulses are used to choose a desired evolution of magnetisation to refocus into a detectable signal.

The constructive interference of coherence pathways is reinforced by experiments showing that, for fixed timing of an experiment, shear rate tests see this rephasing of unwanted magnetisation in different q-steps, all corresponding to the equivalent gradient strength. When possible, the timing of experiments for these gradient strengths were tweaked to negate this effect.

\subsubsection{Image Flow Distortions}

The flow of the sample during an NMR experiment not only has influence on the PGSE component of the experiment but the imaging part as well. Spins encoded by imaging gradients may have moved out of the imaging volume by the time the rephasing occurs. Figure 3.13 shows the distortion in both the image and velocity map for axial images, and which becomes worse for higher shear rates (from equation (2.8)).

In general, little change was made to the image distortion pattern by decreasing the number of phase steps, changing the q-encoding direction (or turning $\mathrm{q}$ gradient off entirely), rotating FOV, and trying different schemes to order k-space in the phase direction (see [60]). The loss of signal in the top and bottom of the image may therefore be due to the time between read gradients or during read rephasing acquisition in which the spins have moved out of their read voxel to a neighbouring one so are not detected. Spins moving perpendicular to read remain in the same magnetic environment so there is signal on the left and right gaps. Yet spins moving parallel to read in the top and bottom gaps travel the most between environments and so are not detected. This is projection onto the read gradient.

For the PCH geometry due to its planar sections, the spins remain in their specific read environment for longer, so the flow distortions to image are only seen in the end cylindrical regions.

\subsubsection{Optimising Timing Parameters}

Considerable time was spent optimising the NMR parameters to improve the performance of an experiment or series of experiments. Careful consideration was applied to the overall length of an experiment with regards to $T_{2}$ relaxation, choice of FOF for expected velocity range, aiming to use lower more stable gradients, and was manageable for stepping through a wide range of shear rates for consecutive 


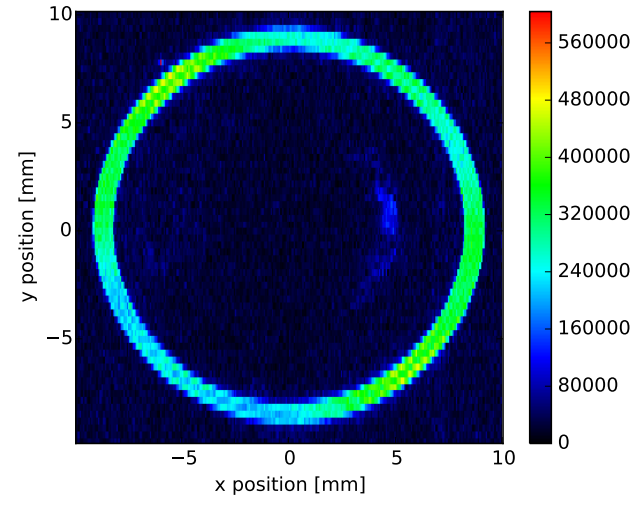

(a)

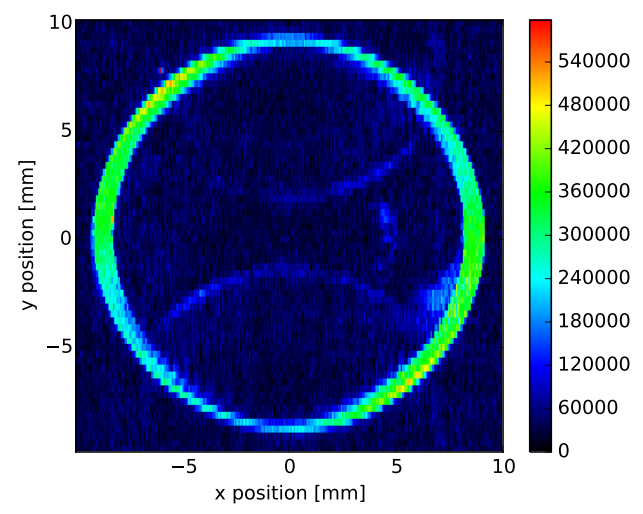

(c)

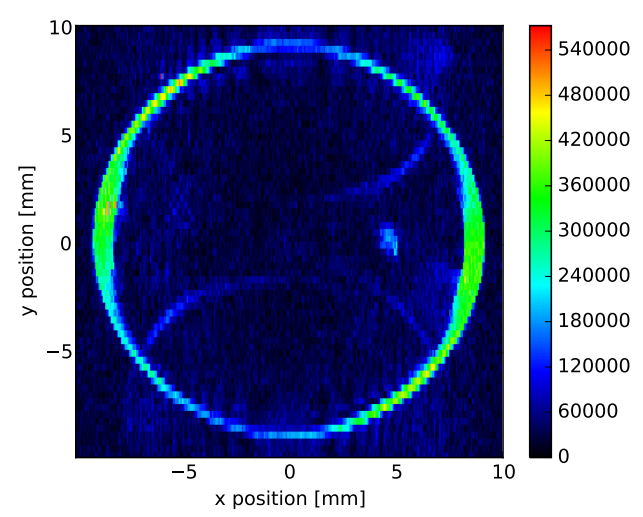

(e)

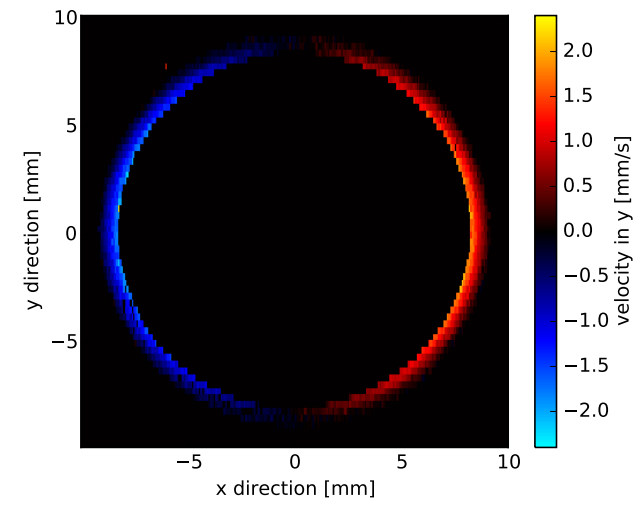

(b)

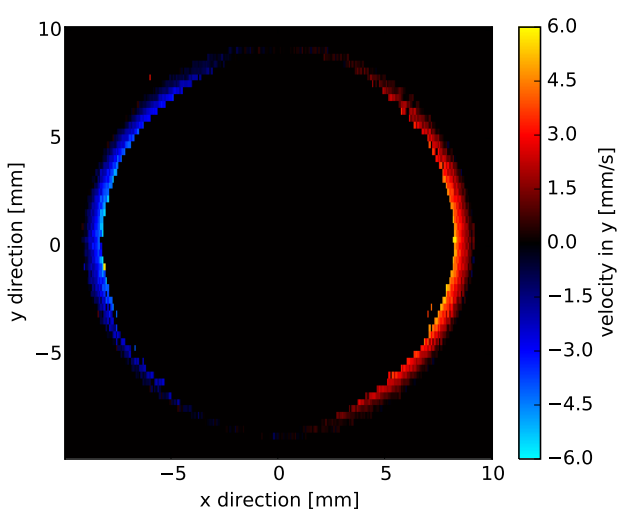

(d)

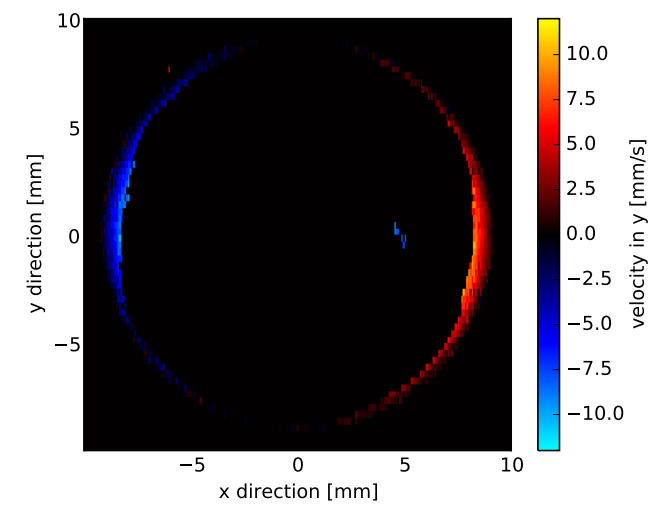

(f)

Figure 3.13: PGSE-RARE plots from TC geometry with water showing flow distortions for shear rates (top to bottom) $\dot{\gamma}=2,5,10 /$ s. Left column is RARE images, right column is the corresponding velocity map. 
experiments with additional rheological timings. An attempt was also made to avoid unwanted magnetisation described in Section 3.3.3. Overall timing, excluding a $5 \times T_{1}$ repetition time, was kept to less than or approximately equal to $T_{2}$, which along with 8 repeat scans, produced adequate signal to noise. The FOFs were taken to be the maximum expected velocity (times two if q-space scheme covered negative q-space) with a buffer of $20-50 \%$ of that value.

Optimising the timing from these constraints uses equation (2.22) for the velocity encoding final phase difference. Nyquist theorem states that this phase must not be equal to $2 \pi$ or exceed it for each gradient increment to avoid ambiguity of the signal. Hence

$$
g_{\max } \approx \frac{2 \pi N}{\gamma \delta \Delta v_{\max }}
$$

where $v_{\max }$ is the FOF and $N$ represents the number of gradient increments to maximum gradient (recall the different functions of $q_{\text {dim }}$ in Section 3.2.2). Displacement encoding described in Section 2.2 assumes that $\delta \ll \Delta$, the time when the encoding gradient is active the spins are 'stationary' relative to how long they are left to move before they are rephased.

Two approaches were taken for the range of shear rates of study. A range over two orders of magnitude used a set upper limit for the gradient for lower shear rate experiments, then kept $\delta$ and $\Delta$ constant for higher shear rates (adjustments for odd $\mathrm{q}$-step experiments). For a range closer to one order of magnitude the displacement, $v \Delta$, measured were kept constant along with the gradient pulse dimensions $\delta$ and $g_{\max }$. 


\section{Chapter 4}

\section{Planar Cylindrical Hybrid (PCH) Geometry Development}

This chapter presents the further development of the novel shear geometry, the planar cylindrical hybrid (PCH), including the methodologies designed for its effective use. The work presented in this thesis picks up from the first realised iteration of this device, introduced in Section 2.4, aiming to address the misalignment issues and improve usability. As part of the entire rheo-NMR set up, the temperature control apparatus was amended to improve control over the temperature inside the magnet. The temperature set up is presented here, along with set up procedures for the geometry (included is the calibration of the NMR imaging system and an exploration into the orientation and flow direction physically captured by the velocimetry experiments).

\subsection{Modifications to Previous Design}

In an effort to realise a shear geometry that could subject a material to planar Couette deformations, a first iteration of a PCH geometry was designed and built (compatible with a rheo-NMR testing environment). For reader clarification an illustration of the design is explored here in Figure 4.1, with individual components labelled $^{1}$. Figure 4.1 also includes the nominal dimensions of the ideal design so that the reader can get a sense of scale.

This iteration can be broken down into two main sections; the cap and the cup. The cup made up the outer wall of the geometry with a racetrack shaped hole. The

\footnotetext{
${ }^{1}$ Note that it has already shown in a photo and in a section of a computer animated design in Figure 2.15.
} 


\section{CAP}

(inner wall component)

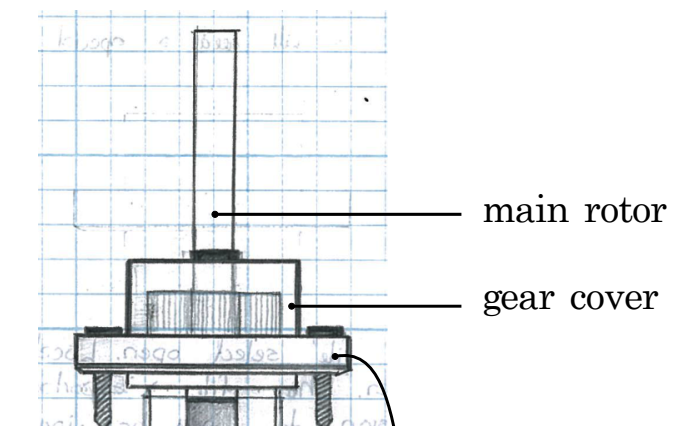

CUP

(outer wall component)
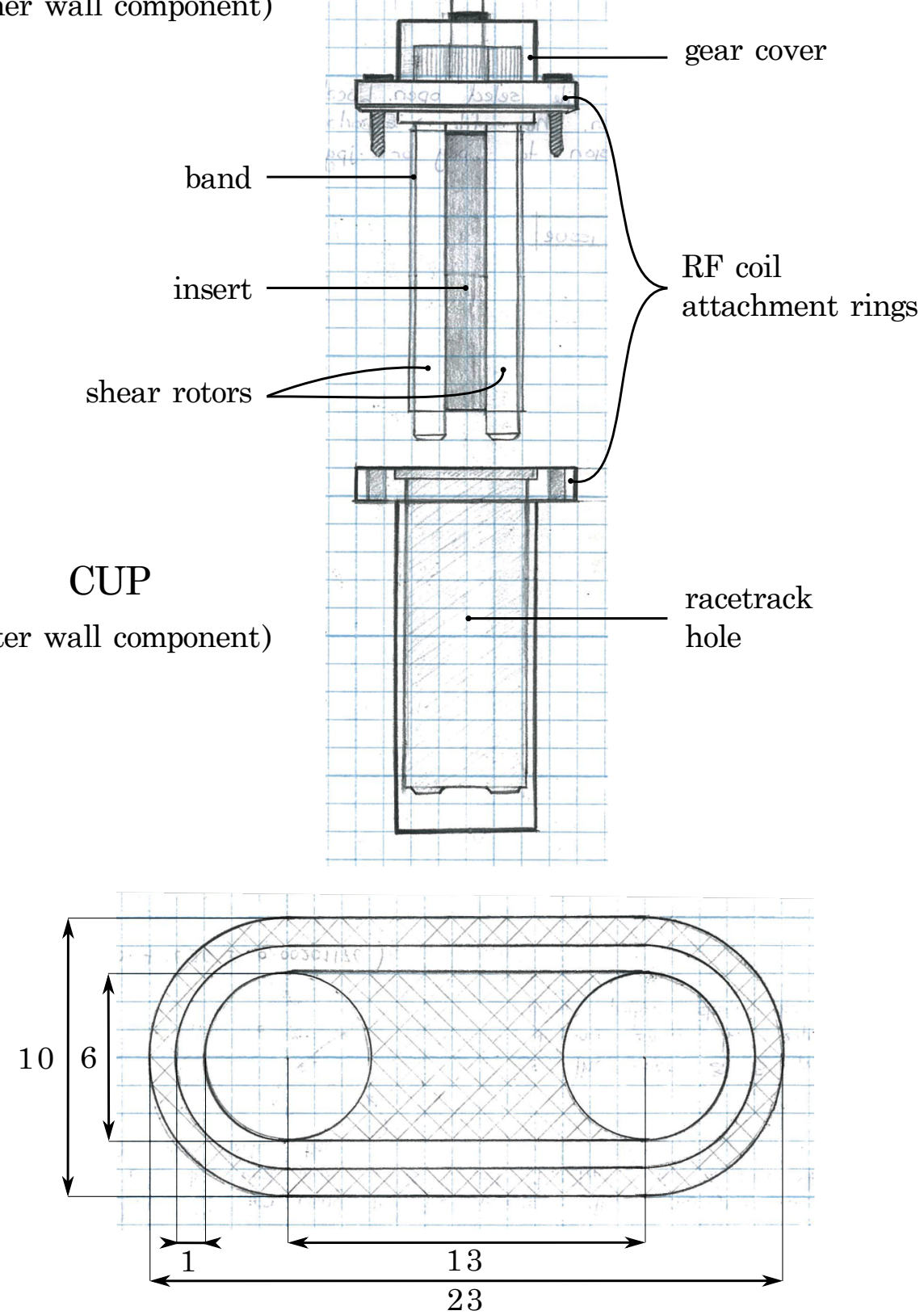

Figure 4.1: Illustration of first iteration of PCH shear geometry with labelled components (top) and nominal dimensions (in $\mathrm{mm}$ ) of the shear geometry with sample area hatched (bottom). The device has be drawn with the cap and cup components separate. 
cap comprised the lid to the cup, topped by a main rotor to connect to an external drive shaft, and the inner wall aspect which applies the shear: two (shear) rotors wrapped in a conveyor-like band (with a potential insert piece to hold rotors apart). The same names are used for similar components in the updated design and any new ones will be introduced when applicable.

The purpose of the second iteration of the design is to address issues with the first device, as well as make enhancements so it is more effective in achieving its aim. The alignment / dimensions of the inner wall components, in how they relate to the uniformity of gap width, needs improving. Additionally the geometry can be adapted to assist with better temperature control of the sample, as well as in how it fits (a) together and (b) with the NMR system.

\subsubsection{Plug - Alignment of Rotors}

One issue with the first iteration of the $\mathrm{PCH}$ geometry was in the misalignment of the holes at the base of the geometry cup racetrack hole used to align and stabilise the rotors. The holes work against the tension of the band in an attempt to keep the rotors vertically straight. An axial MRI image with four slices at different heights, Figure 4.2, shows a twisting of the band which results for the misalignment of the rotor holes.

This misalignment is significant when compared to the thickness of the gap, where uniformity of the gap is necessary to obtain truly planar regions of flow. It is important to note that the left planar gap appears straighter only because the position of the rotors has forced the insert to be rotated and not in proper connection with the rotors (coincidentally compensating for the bottom rotor in the image not being far enough to the left). The degree of twisting of the rotors due to the misalignment changes as a function of height. As such, the solution for previous studies with this iteration, was to twist the top cap to minimise the twisting effect in the imaging volume. However this will still result twisting above and below the imaging volume which could introduce unwanted vertical flows.

Mechanically the rotor holes were difficult to drill. To assist with this in the updated version of the design the cup component is separated into two pieces, a tube and a plug. The 'tube' is similar to the cup with the racetrack hole milled through the entire piece, while the plug provides the floor of the sample volume. This allows unrestricted access to the surface where the rotor end holes will be cut out, improving the likelihood of mechanical accuracy. 


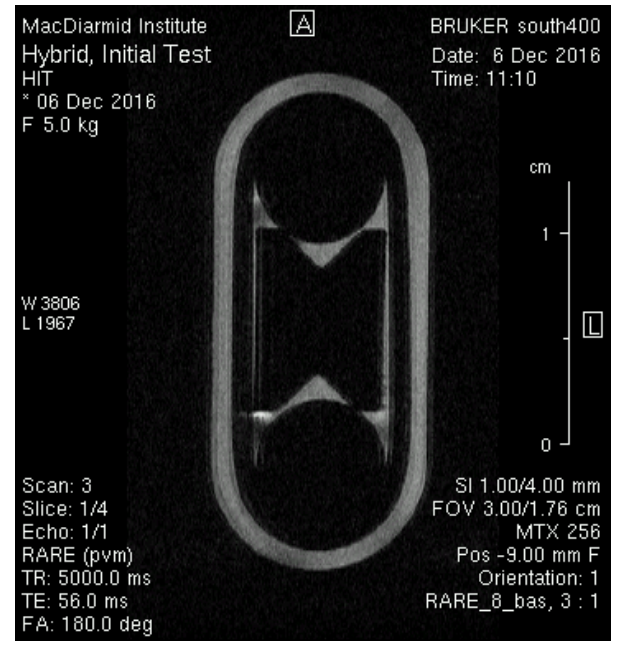

(a)

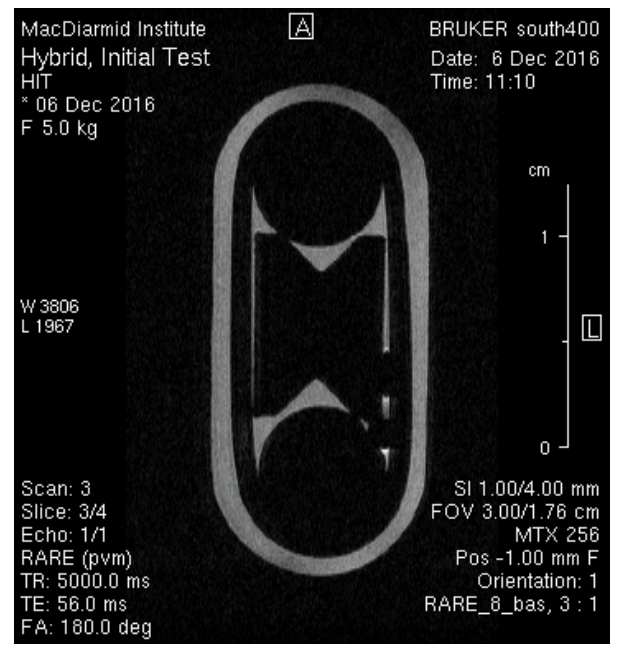

(c)

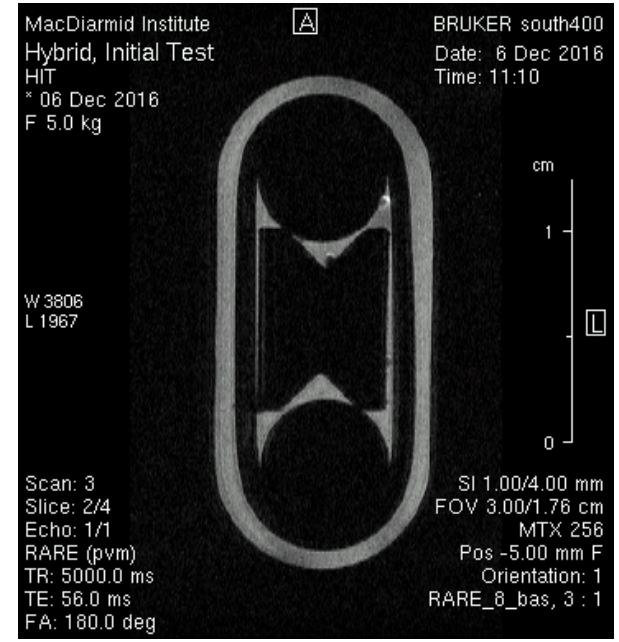

(b)

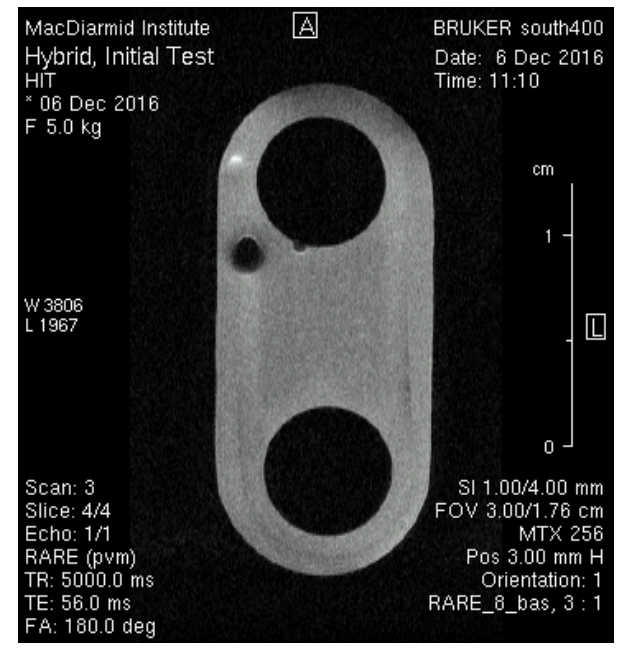

(d)

Figure 4.2: Axial MRIs of the first iteration of the PCH. Slices are $1 \mathrm{~mm}$ thick and evenly spaced to span $17 \mathrm{~mm}$ in $z$ (a) top to (d) bottom. (a-c) Show the insert (center), two circular rotors and the band wrapped around them. (d) is below the height of the band and insert so the misalignment of the holes is visible. 


\section{Features}

The plug was a flat disk with a raised piece the shape of the racetrack, Figure 4.3. The raised racetrack shaped piece was designed to have a locating fit with the racetrack hole in the tube piece. This helps accurately align the plug and hence the new rotor holes in the base of the sample environment, relative to the outer wall of the geometry.

Additionally the shape of the rotor holes were changed to a tapered shape, with the end of the rotors themselves made to match. The only contact between the holes and rotor is now at the sloped sides. This tapered fit assists with alignment and a repeatable fit between these two pieces is easier to confirm with MRIs. Sample fluid will need to be pushed out of the tapers to accommodate the rotors (sample added before the cap piece can be inserted), but due to the tight stabilising fit wanted between the two surface this is not possible. Small grooves were cut in the sides of the tapers, as shown, to allow for this.

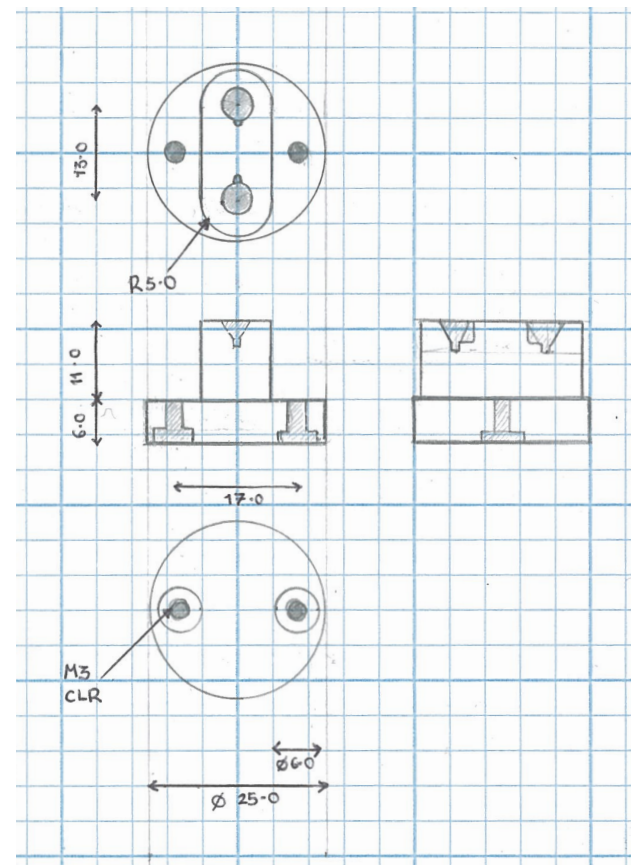

(a)

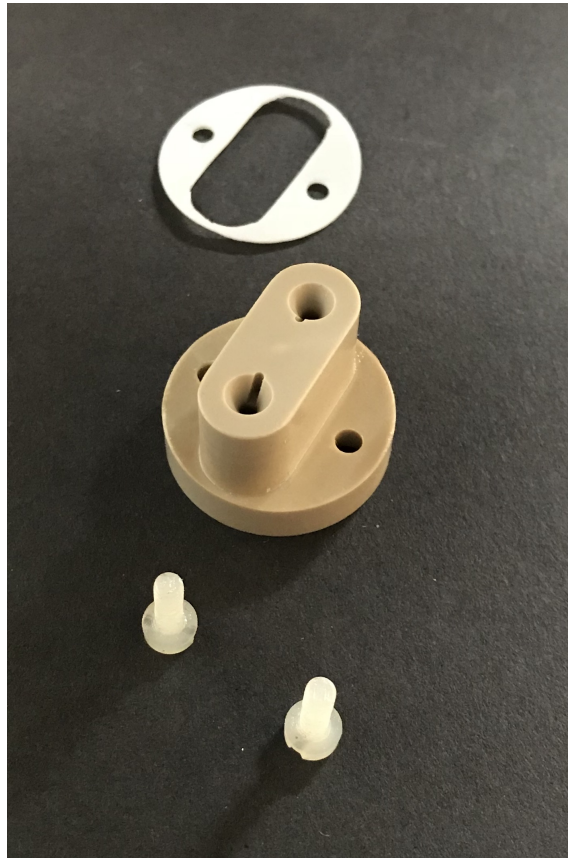

(b)

Figure 4.3: (a) Diagram showing top, bottom and side views of plug piece, with dimensions. (b) Plug components, from top down: PTFE face seal, PEEK plug, M3 Nylon base screws (connects to tube piece). 


\section{Attachment to Tube}

It was important for the practicality of the design to have a flat base so the assembled geometry could stand unaided on a benchtop. Consequently the base screws which pull the plug into the base of the tube have been countersunk, Figure 4.3a.

An earlier idea had been to have an o-ring to create the seal between the tube and plug, like in rheo-NMR TC devices. However this o-ring groove was not doable with the current workshop equipment. The solution was instead to have a Teflon face seal (top in Figure 4.3b) which sits between the two pieces. This leaked so a layer of Vaseline was used between the plug and face seal. 10 turns of the screws reaches the point of resistance which, along with the Vaseline and face seal, provides a watertight seal, and puts the sample floor at a repeatable height. The Vaseline section lies outside of the imaging FOV.

\subsubsection{Band Elastomer}

The band is a key component in the geometry as its flexibility allows for straight walled shearing and a flow field with closed streamlines. It is mixed from a twopart Sylgard 184 Silicone elastomer kit poured into a custom built mould made to dimensions to allow the band to be under tension while around the rotors (see [4] for image and description of process). The mould is cylindrical to prevent any memory shape in the elastomer or unevenness in the band structure when it is rotating.

Trimming of the band was required at both ends make the band $\approx 45 \mathrm{~mm}$ in length. This was tricky to do with a craft knife to achieve a straight end and avoid tiny nicks that could turn into large tears when under tension. To help this, two additional approaches were tried. The first was to use an o-ring to block off the lower end of the cylindrical mould to prevent the thinner layer that would form where the inner piece of the mould was sitting in the base of the mold case. This had mixed success as the o-ring satisfied this but the elastomer material set slightly to the o-ring itself. This resulted in a sticky end which was not noticeably less uneven than craft knife cutting attempts. Secondly the amount of elastomer poured into the mould was reduced to give a self seal end at the top of the mould ${ }^{2}$.

What was explored as part of the geometry development was the extent that the band was NMR active. As can be seen in Figure 4.2, the band appears NMR transparent in the MRIs. To further confirm this, a small amount of band material was

\footnotetext{
${ }^{2}$ For future use of the mould it is recommended to make mixtures of at least twice the amount required $(\approx 2.22 \pm 0.11 \mathrm{~g}$ from mould dimensions and density of elastomer base) as roughly $2 \mathrm{~g}$ can be lost in the transfer process to the mould and another $0.3-0.4 \mathrm{~g}$ sets on the mould funnel.
} 
allowed to cure in a separate $5 \mathrm{~mm}$ NMR tube and its relaxation times were found, $T_{1} \approx 1.4 \mathrm{~s}$ and $T_{2} \approx 10 \mathrm{~ms}$. The spin-spin relaxation time was additionally extracted from WLM in PCH tests using a non-negative least squares mathematical optimisation macro in Prospa. These were in agreement, with the band component of the $T_{2}$ decay contributing approximately $10 \%$ to the total $T_{2}$ decay curve. Equivalently for doped water in the PCH geometry the $T_{2}$ of the band was not significantly 'visible'. Very short $T_{2}$ s like $T_{2} \approx 10 \mathrm{~ms}$ are ideal for the study in this work as both MRI and velocimetry imaging experiments used are based on a RARE sequence. As eluded to earlier this means that the band signal will have easily decayed before it can be detected. Therefore it should not influence the data presented later in this thesis.

The importance of how the band is put on the shear rotors will be left to Section 4.3.1 as this discusses the set up and use of the geometry.

\subsubsection{Interaction with RF coil}

The RF coil is a component in the NMR system which houses the sample, and transmits and receives the NMR signal. Two improvements were made concerning the interaction between the $\mathrm{PCH}$ geometry and the coil:

\section{Aligned Attaching}

Alignment of the inner wall and outer wall in the sample region of the geometry and how vertical the whole device is sitting relative to the RF coil, and hence the imaging gradients, are both important. The former ensures a repeatable and desired flow environment, while the latter makes the imaging and velocity encoding process easier as vertical rotations of the $r, p, s$ coordinates to do not need to be factored into experiments.

The alignment can be controlled by the four screw holes on the top and bottom RF coil attachment rings (cap and cup respectively) visible in older version of the device in Figure $2.15 c^{3}$. The holes are arranged in a square, however as in Figure $4.4 \mathrm{a}$ the threaded holes on the top of the RF coil are in a rectangular formation. As such only one diagonal pair of holes on the $\mathrm{PCH}$ rings will be used to attach the geometry to the RF coil. For this diagonal pair both ring layers have the screw holes drilled out (the force between the base of the screw head on the ring's top surface and the catching of the thread in the RF coil) which holds the geometry in place. In contrast for the other diagonal holes, only the top ring layer is drilled out

\footnotetext{
${ }^{3}$ More than four holes can be seen in this image. The two holes closest to the center front and center back of the cup ring were extra holes drilled as part of the design process and should be ignored.
} 
(meaning the force is now between the screw head on the top ring surface and the catching thread on the bottom ring), holding the cup and cap together and controlling flow environment. Having only one set of threaded holes in each case prevents the occurrence threads not lining up between adjacent layers, which would prevent a flush surface interaction and deform the screws. M3 nylon screws were trimmed to a thread length $17 \mathrm{~mm}$ and $11 \mathrm{~mm}$ were used respectively. They were threaded until no light was visible between the three connecting layers. The two types of attachments are shown in Figure 4.4b.

\section{Cooling Gas Flow}

Rheological behaviour is highly temperature dependent ${ }^{4}$. Berret et al. [63] shows that the onset of shear banding of a CPCl-NaSal WLM solution, similar to the sample used in this thesis, shifts by a magnitude in onset shear rate over a change of $10{ }^{\circ} \mathrm{C}$. Section 4.2 addresses this from a NMR system point of view while here it is approached in terms of geometry design.

As part of the NMR temperature control system cold gas is pushed around the geometry up through the bore hole of the magnet. Figure 4.4a shows holes in the black layer of the RF coil which assist this flow. While the tube and plug component of the geometry cannot be easily carved into the gas flow around the geometry can be improved by making divots in the ring layers of the cap and tube. This means that the flow is not blocked off at the top RF coil by the rings. Due to the sloped walls of the black layer, the placement of these divots are not important. Divots were made approximately semicircular, of diameter $5 \mathrm{~mm}$, to approach the same cross-section surface area as the gas delivery tube inside the magnet probe. These are illustrated in Figure $4.4 \mathrm{c}-4.4 \mathrm{~d}$.

\subsubsection{Improved PCH Device}

Implementing all these improvements results in the second iteration of the $\mathrm{PCH}$ shear geometry, and the one which shall be used to study a complex material in the following chapter. The components are shown in Figure 4.5 along with MRIs showing the main slice orientations. The readers attention is drawn to the center MRI and the improvement in the alignment of the rotors. A dot may be noticed in the axial (center) MRI, off to the left of the shear environment. This is the marker dot used to help orient the geometry for experiments (discussed in detail in Section 4.3.4).

\footnotetext{
${ }^{4}$ Consider that most everyday materials become runnier (less viscous) when heated.
} 


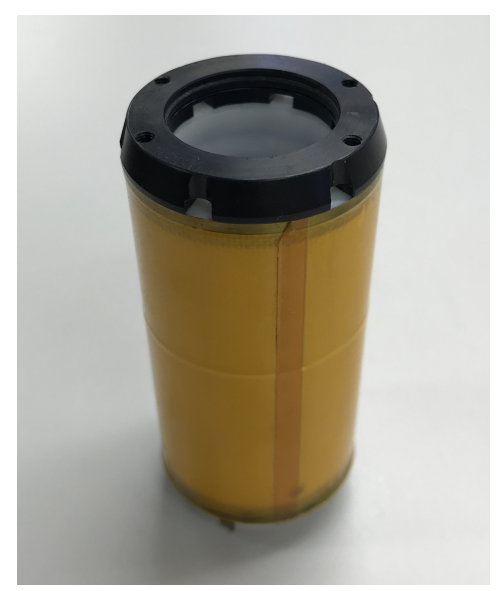

(a)

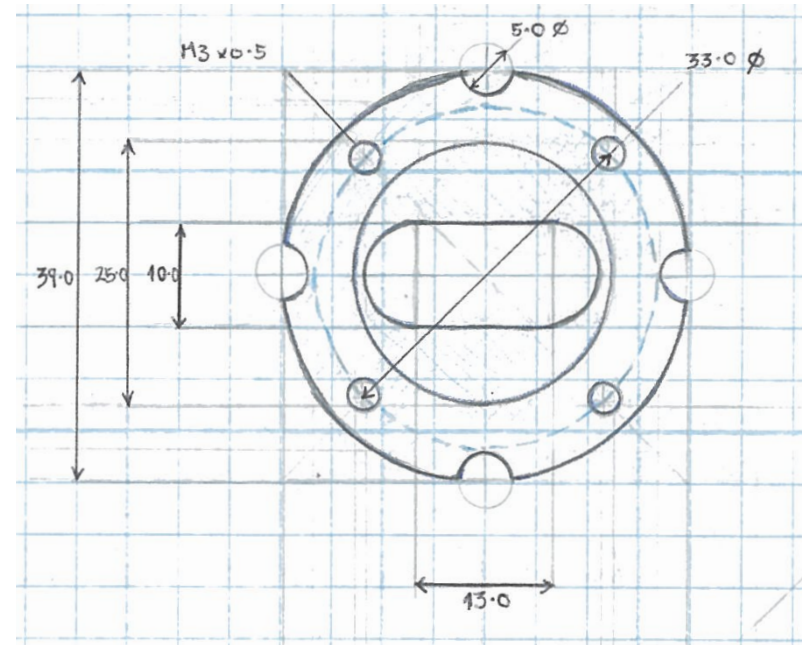

(c)

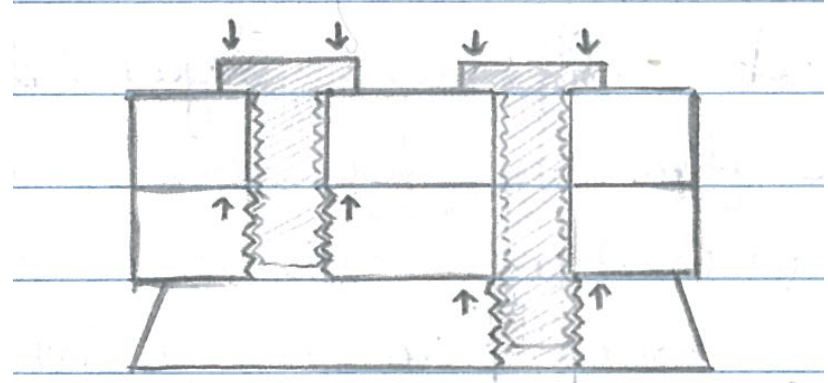

(b)

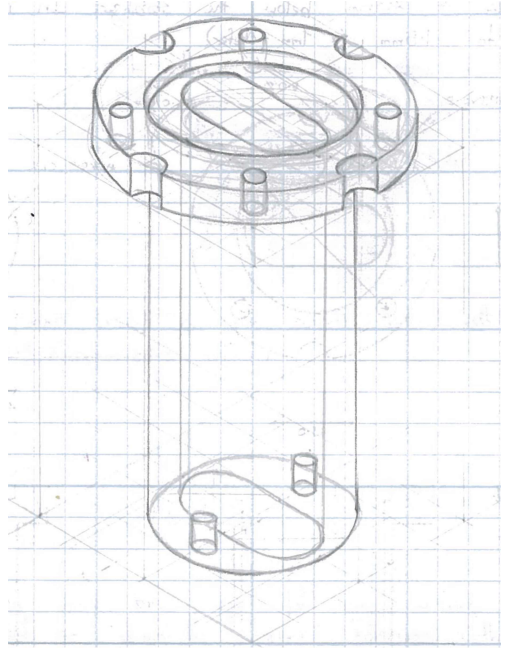

(d)

Figure 4.4: (a) Top of a $25 \mathrm{~mm}$ RF coil, black section has slopped walls and gas flow holes. (b) Two screw types for attaching the top and bottom RF coil attachment rings of the $\mathrm{PCH}$ and the top of the RF coil. (left screw) Only bottom ring is threaded so screw head and this threaded hole holds two ring layers together. (right screw) Both rings drilled out so screw head and thread on RF coil hold geometry onto the coil. (c, d) Technical drawing of bottom RF coil attachment ring on $\mathrm{PCH}$. Top ring has the same outer pattern. 


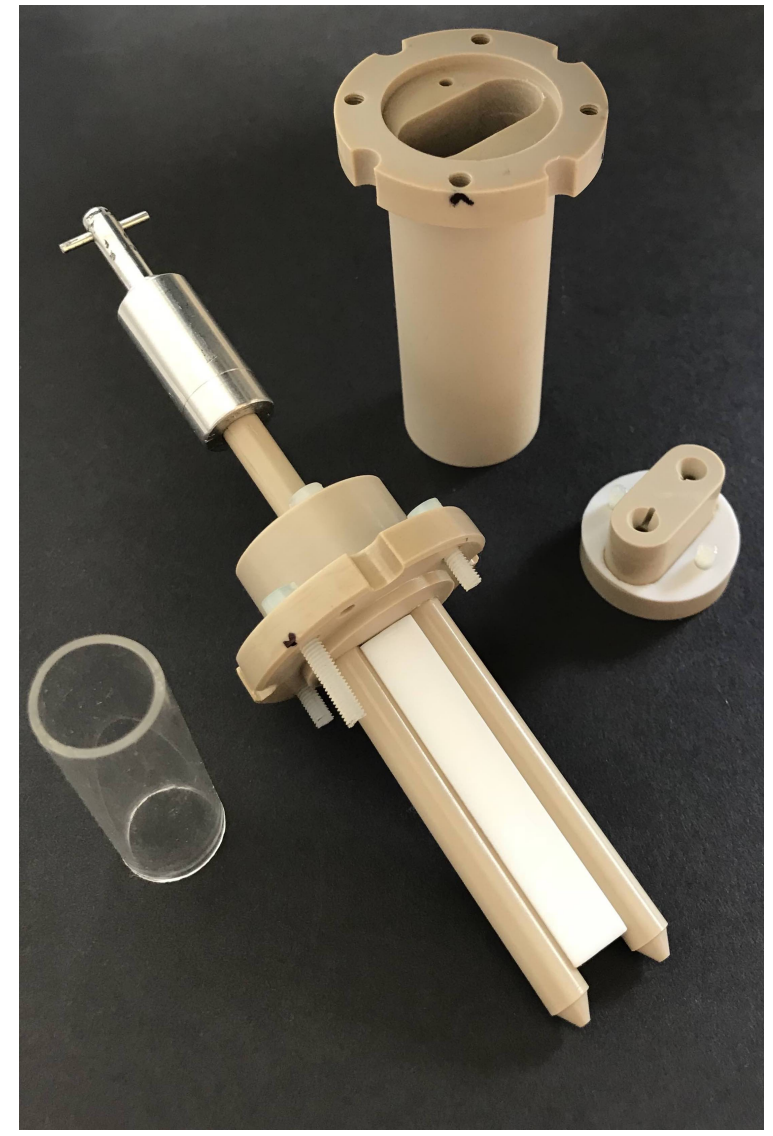

(a)

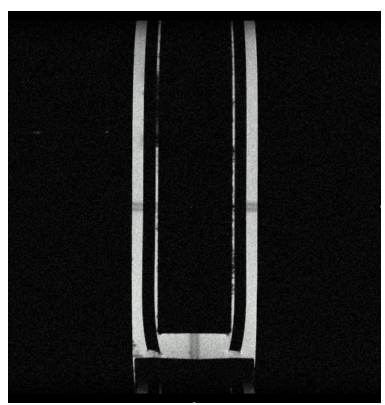

(b)

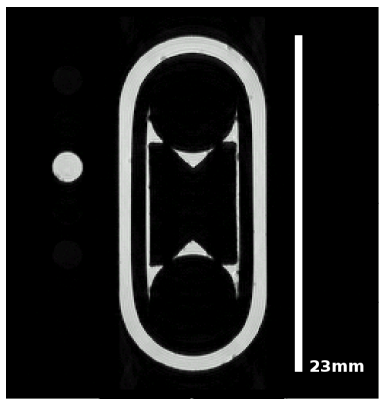

(c)

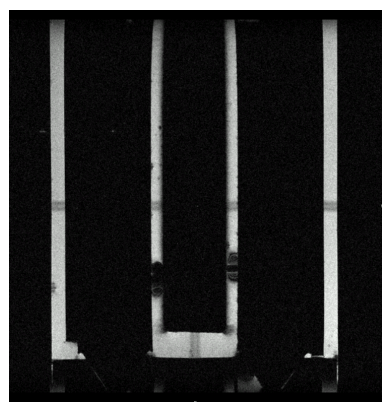

(d)

Figure 4.5: Version of Planar Cylindrical Hybrid developed in this these. (a) Partly disassembled components showing the band, cap, tube and plug. (b-d) MRIs showing the three main orientations: planar, axial, and cylindrical slices. 


\subsection{Temperature Control for NMR System}

As mentioned previously, controlling the temperature of a sample during rheological testing is key. The NMR Spectrometer used in this thesis was equipped with a Bruker Variable Temperature Unit (VTU) to assist with this. It controls temperature in the sample region of the magnet by pairing a gas supply (dry compressed air for above room temperature, boil-off from a liquid nitrogen dewar for below), a heater, and a thermocouple near the sample to provide feedback. A probe head dewar (thin double walled silvered tube) sits inside the magnet probe and delivers the gas to the base of the sample while also housing the heater coil. The gas supply flow rate is fixed and its temperature is determined by the source, hence the heat exchange with the sample is controlled by the heater and PID parameters determined in Topspin.

The desired temperature for experiments in this work was $25^{\circ} \mathrm{C}$ as this aligns with other WLM experiments and rheometer measurements. Using boiled off liquid nitrogen as a gas supply is an additional hassle and is over-kill for the temperature required. To get a significant (at least $10^{\circ} \mathrm{C}$ ) temperature difference between the desired $25^{\circ} \mathrm{C}$ and the cooling gas temperature a A60015A Adjustable Coldstream Air Gun by Meech was connected to cool the building supply compressed air (which typically sits around room temperature but is weather dependent). The degree of cooling is related to the cold fraction (percentage of input air released out the cold end of the Coldstream Air Gun), which is quoted as achievable up to $70 \%$ at 5.4 bar and up to a $50^{\circ} \mathrm{C}$ cooling of the gas. As Coldstream Air Gun is incredible noisy approaching this pressure and that level of cooling was not required, an input pressure of 1.5 bar with the cold fraction control knob at $3 / 4$ turn (with lowest flow rate insert) was found to be sufficient. Measuring the output over the course of three hours (on order of range of shear rate experiments) with a thermocouple the temperature, was found to fluctuate around $11.9 \pm 0.7^{\circ} \mathrm{C}$, approximately $13^{\circ} \mathrm{C}$ below the desired temperature.

The building supply air was tapped into near the magnet with a ' $\mathrm{T}$ ' junction and valve, then connected through a pressure regulator to the Air Gun. In Figure 4.6 the Coldstream Air Gun can be seen cushioned by a foam block and zip-tied to a leg of the magnet housing. From careful testing, by gripping the Air Gun and manually moving it up and down below the bore of the NMR magnet, it was found to produce slight eddy currents when moved in this magnet field. However the device was not attracted towards the NMR magnet. Consequently it is safe to use the position in Figure 4.6 for the Air Gun. Flexi grey tubing that came with the device channeled the cold air output into the opening of the probe head dewar 


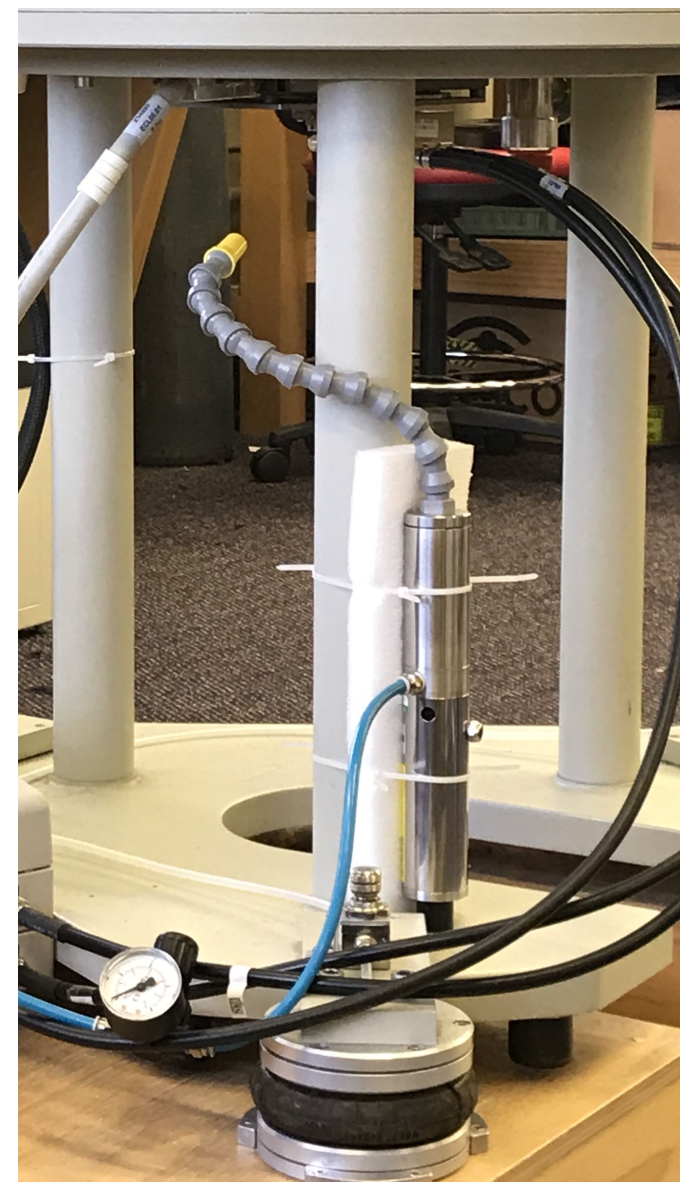

Figure 4.6: Coldstream Air Gun assembly at NMR magnet (not connected).

with the use of Teflon tape to create a stable fit.

This temperature control set up was sufficient for the experiments dealt with in this thesis. The set up is now additionally available for controlling other NMR experiments which require temperature below room temperature.

\subsection{PCH Preparation and Use}

This section outlines how a rheo-NMR practitioner would interact with the device, from assembly and sample loading, to pre-experiment MRI checks. To prepare the NMR system for experiments the imaging gradients must be properly calibrated and the user should have a good understanding of the image and flow orientation link between the physical set up and the processed NMR data. An explanation on how these were done for the magnet system used here are included. 


\subsubsection{Assembly}

The PCH geometry needs to be deconstructed and cleaned before sample loading. Once dry, the plug was assembled and attached to the tube as in Section 4.1.1, and any excess Vaseline wiped off. To move the band onto the rotors both surfaces needed to be lubricated with water and detergent. The band can then be slid on, with a couple of mms left between the end of the band and the start of the rotor tapers. The insert was forced in at this stage so the band would be under even distortion and less likely to tear. Massaging the band as water was being used to wash off the detergent was necessary to ensure even tension (and therefore thickness) throughout the circuit of the band. Compressed air, not heat, removed the water from the geometry.

From the nominal dimensions of the design, a sample volume was calculated to be between $2.5-5.5 \mathrm{~mL}$ depending on whether the insert was used or not, and the specific height of the band used. The doped water sample does not have a shear memory and has a low viscosity so could be syringed into the racetrack hole. For the WLM sample obtaining the correct volume was done by weight and carefully poured into the hole, in a darkened room to protect the sample $e^{5}$. Next the inner wall component of the shear geometry was inserted into the tube, and secured with one diagonal set of ring screws. Care must be taken with more viscous samples, and the cap lowered slowly to prevent material being forced out the top of the geometry. The grooves cut in the attachment rings for the cooling gas to flow act as rotational alignment guides for aligning the cap and tube together.

The final set of the screws go through the gear cover. These will determine how the rotors fit into the tapers. A series of images (ParaVision), Figure 4.7, were taken of cylindrical slices with the geometry raised in the RF coil so the base of the sample environment was centered. From these it was determined that $93 / 4$ turns was the first discernible point (to the nearest quarter turn) where complete contact between the rotors and tapers were made.

If the rotors were pushed down too far they may start to buckle or bend, and if not pushed down enough the rotors may be pulled too close together and the band bows in the planar region, Figure 4.7. In that case there is also more freedom for mechanical wobble when the rotors are rotated. Both these extremes will cause inhomogeneities in the flow field which should be minimised.

\footnotetext{
${ }^{5} \mathrm{~A}$ rough estimate of the density of the WLM solution was made by filling a measuring cylinder and letting it settle to measure the volume and added weight. Assuming the evaporation was negligible it was tentatively found to have a density similar to that of water.
} 

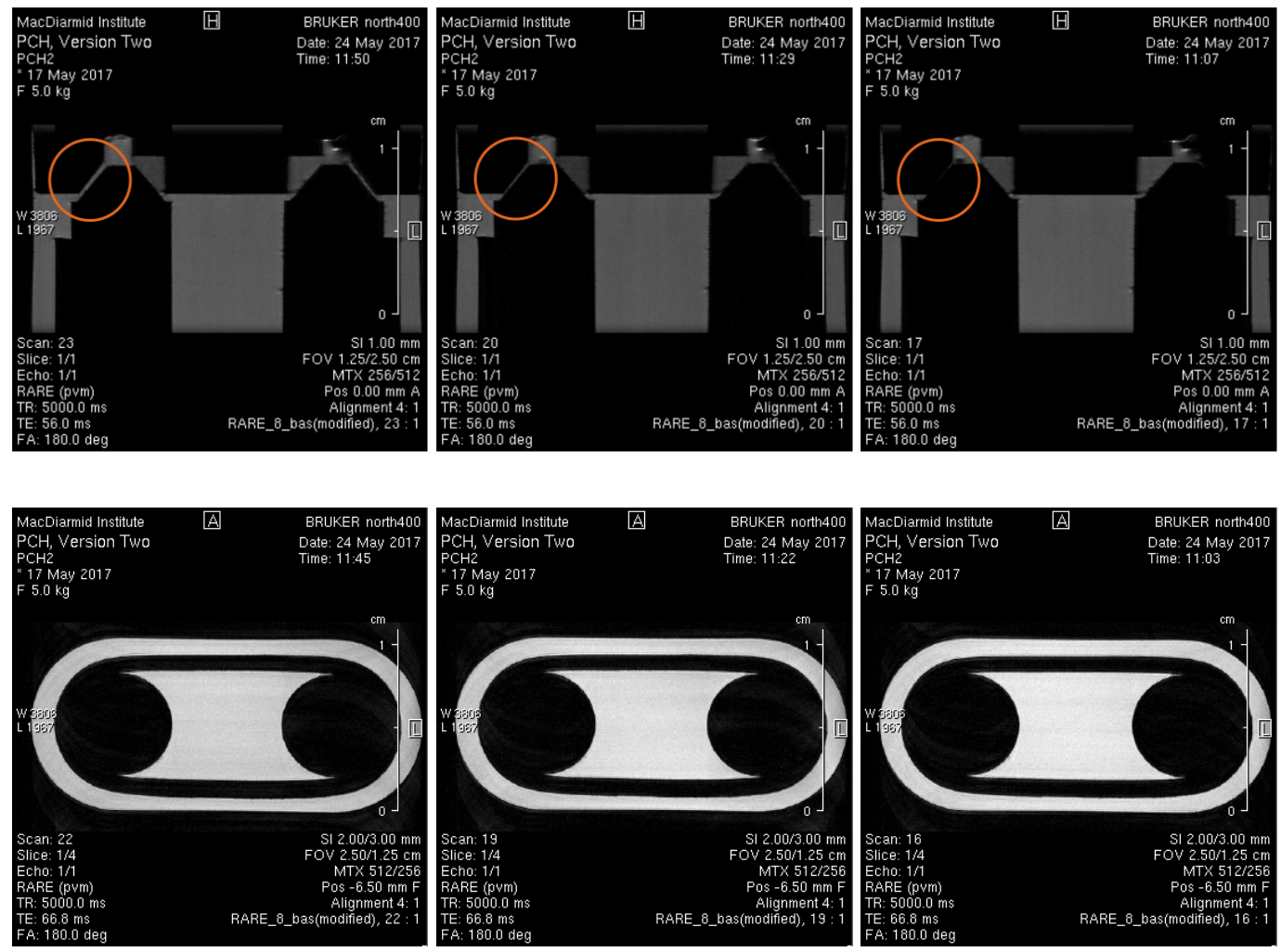

(a) $91 / 4$ turns

(b) $91 / 2$ turns

(c) $93 / 4$ turns

Figure 4.7: MRIs of the PCH geometry studying the effect of the number of turns of the gear cover screws on the fit between rotor ends and tapered holes. (top) Cylindrical slices centered around the base of the sample region (view upside down). (bottom) Axial slices to compare corresponding band shapes $8 \mathrm{~mm}$ up from base. The orange ring highlights one of the contact points.

The Teflon insert was designed in the original device iteration, however not used for experiments. As the changes in the new version have improved the alignment of the rotors the insert is now able to sit symmetrically between the two rotors and further stabilise their movement. The insert additionally keeps the rotors evenly separated in the vertical direction, compensating for the looser holes in the cap ring from which the rotor protrude (as this is a higher rotating part the fit requires more tolerance). The comparison Figure 4.8 shows the reduction of bowing of the band after the introduction of the insert piece. Consequently it will be used in all experiments henceforth.

For rheo-NMR experiments, the main rotor of the $\mathrm{PCH}$ is clasped by a spindle (similar to that on the TC geometry in Figure 2.14a) and the geometry is secured 


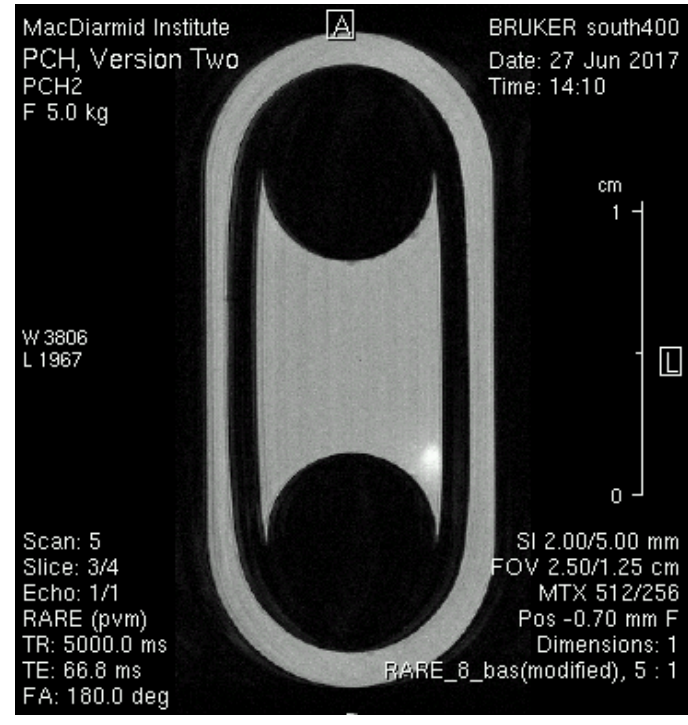

(a)

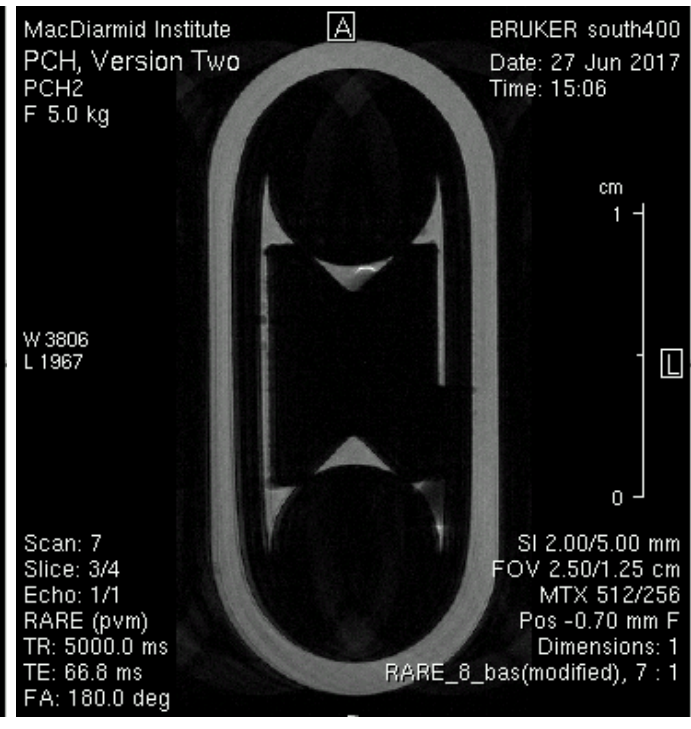

(b)

Figure 4.8: MRI axial slice of PCH geometry (a) without and (b) with stabilising insert.

to the RF coil (refer to Section 4.1.3). Once mounted to the probe and inserted into the magnet bore, the temperature control system can be attached. Lowered in from the top is the rheo-NMR drive shaft and motor, which is then connected to the controller unit box sitting on the operation desk of the NMR spectrometer. Through the network connection the drive shaft can be controlled via a web interface. This is geometry independent so frequencies are used (which can be converted into shear rates as discussed Section 5.3.2).

\subsubsection{Imaging Gradients Calibration}

The accuracy of both NMR imaging and velocimetry hinges on the gradient coils being correctly calibrated (object imaging dimensions need to match physical dimensions). This calibration requires a well defined and precise object to measure. Ideally this should be on the scale that the real imaging is interested in, to account for slight unintended spatial variations of the field. Both a standard imaging sample and the TC geometry were ruled out due to poor signal-to-noise and size of dimension measured (center pieces too large to easily pixel count measure in ParaVision).

Instead the criteria can be met by the rotors in the $\mathrm{PCH}$ geometry, which are clearly on an appropriate scale, and measure $6.00-6.01 \mathrm{~mm}$ in diameter (with 
Vernier digital calipers). A total of 32 data sets were taken per imaging coil direction $(x$ or $y)$ :

- 2 rotors per image

- 4 angle positions of the geometry in the $x y$ plane, each approximately separated by a quarter turn,

- 4 slices per scan.

Under recommendation of the micro-imaging manual only phase distances were measured $^{6}$. The average diameter and error were established from these 32 diameters. Scaling these to the known physical dimensions obtains a calibration factor that can be entered into the gradient scaling factors in each respective program used (i.e. TopSpin and ParaVision) as per manual. The scaling for $x$ and $y$ were in approximate agreement so the average was taken an applied to the gradient calibration constant to get a new value of $642936 \mathrm{~Hz} / \mathrm{cm}^{7}$. On single repeat image comparisons, the calibration reduced the average dimension over four slices to less than $0.7 \%$ with any single point variation less than $2 \%$ (from $3.3 \%$ and $4.2 \%$ ).

\subsubsection{Set Up Checks}

In preparation for using the $\mathrm{PCH}$ geometry for flow experiments, MRIs were used to perform pre-experiment checks to determine proper set up. Included in this was an investigation of the rotational alignment and vertical straightness of the geometry sitting in the RF coil with respect to the imaging gradients, the uniformity of the sample (lack of bubbles), and that the rotors are sitting in the tapered holes. The experiments (in terms of types of slices) and their purpose are summarised in Table 4.1. All images were done with equal or better resolution than the intended velocimetry tests so any small scale features in the velocity maps would also be resolved here.

For convenience these are done in ParaVision which requires first a set of three orthogonal slices to act as a reference frame for aligning all later slices. Figures $4.5 \mathrm{~b}$ to $4.5 \mathrm{~d}$ form a single example of this (in the special case where the symmetry axes of the geometry already physically lined up with the imaging gradient coordinates). From there a series of axial slices can be taken at different heights. The axial slices work well to confirm or deny the correct angle rotation in the $x y$ plane and will also show image distortions in the locations of bubbles in the sample. A cylindrical

\footnotetext{
${ }^{6}$ The focus here was on the $x y$ plane however a similar procedure can be used to calibrate $z$.

${ }^{7} 15.1009 \mathrm{G} / \mathrm{mm}$
} 


\begin{tabular}{|c|c|c|c|c|c|}
\hline 炁 & $\begin{array}{c}\text { Three } \\
\text { Orthogonal }\end{array}$ & Axial ( $z$ ) & $\begin{array}{c}\text { Lower } \\
\text { Cylindrical }\end{array}$ & Cylindrical & Planar \\
\hline $\begin{array}{l}0 \\
\infty \\
0 \\
0 \\
\vdots \\
\vdots\end{array}$ & $\begin{array}{l}\text { basis for } \\
\text { alignment }\end{array}$ & $\begin{array}{c}\text { bubbles, } \\
\text { angle, } \\
\text { dimensions }\end{array}$ & $\begin{array}{c}\text { rotors in } \\
\text { tapers }\end{array}$ & $\begin{array}{r}\text { vertic } \\
\text { alignm } \\
\text { dimensi }\end{array}$ & \\
\hline
\end{tabular}

Table 4.1: Set of preparation MRI experiments for $\mathrm{PCH}$ and what each one checks.

slice with the image offset in $z$ to capture the base of the geometry region checks that the rotors are sitting in the tapers like in the top panel of Figure 4.7c. Bubbles can then be removed through alternating shearing of the sample at $2 \mathrm{~Hz}$ for 5 mins and repeating the series of axial slices, until no bubbles were present in the sample region and also removed from the band / rotor / insert gaps as much as possible. As viscoelastic materials may have a deformation memory, the geometry was removed, Parafilmed to reduce chance of evaporation, and placed in a drying cabinet set at $30^{\circ} \mathrm{C}$ for several hours or over night to allow the sample to relax.

Before flow experiments these tests were repeated, minus the tapers check, and further planar and cylindrical slices were taken, Figure $4.5 \mathrm{~b}$ and Figure $4.5 \mathrm{~d}$ respectively. This confirmed the rotation angle in the $x y$ plane, and the vertical straightness of the two main non-axial slices, used in velocity experiments. To make later experiments easier, the probe was manually rotated and aligned with imaging axes. The angle was made to the nearest half degree, and vertical straightness over $10 \mathrm{~mm}$ in $z$ was deemed acceptable if approximately $\leq 100 \mu \mathrm{m}$. These MRIs additionally can be used to evaluate the gap dimensionality in the sample, which will be discussed in Section 5.3.2.

\subsubsection{Orientation and Flow}

While it is not necessary to consider for the azimuthal symmetric shear geometries typically used in rheo-NMR, the break in the flow symmetry of the novel geometry means that an understanding of the relationship for orientation and flow direction between the processed NMR data and the physical system is critical. Specifically it is necessary to know where the in flow and out flow of the planar regions are. To establish the mapping between physical and image space, two physical indicators must be present, a flow marker and an orientation marker, as well as a NMR gradient marker. 
The flow marker must indicate the mechanical direction of rotation. This rotation is controlled by the drive shaft which itself is driven by a motor. The sign of the user input (to the motor) was calibrated against the direction of the resulting motion by a physical indicator on the shaft. This was a blue flag, a portion of which can be seen in Figure 2.14a. It revealed that positive inputs translate to a clockwise rotation (viewed from above).

The orientation marker needs to be a truly asymmetrical feature within a FOV of the geometry when imaged. The asymmetry allows for up and down, as well as left and right to be established in the images. As it needed to be an NMR active feature, the simplest case is a drilled hole filled with doped water. To prevent image aliasing artifacts it was first considered to have the hole in the Teflon insert, however due to its material and size there was a high risk that the drilling could warp the insert. As such the marker hole was drilled to one side of the planar region, slightly shifted from the center of the planar region (giving right and left). It was made to extend beyond what would be the center of imaging space for the RF coil used but not so far that a $10 \mathrm{~mm}$ or $12.8 \mathrm{~mm}$ FOV in $z$ could not capture the end (giving top and bottom). A top view of its location is Figure 4.9.

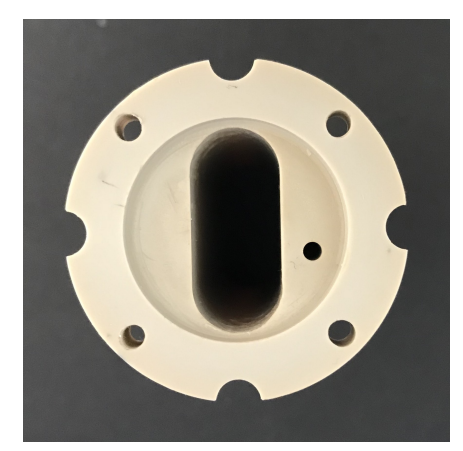

Figure 4.9: Orientation and flow marker hole is right of racetrack hole and below center to put it in an asymmetrical position.

The NMR gradient marker must match the physical directions of the gradients $(x, y, z)$ to the pulse sequence directions $(r, p, s)$ such that the PGSE (velocity) gradients can be observed. In particular, it is important to see the axis that the PGSE gradient pair align with, and whether they step through q-space from a positive to negative or a negative to positive gradient value. The geometry was manually aligned with gradient axes to make this simpler. To 'see' this a oscilloscope was attached onto each of the gradient amplifiers and screen shots were taken near the start and end time of each pulse sequence. 
To determine the orientation and flow one axial, three planar, and three cylindrical slices were taken with the same clockwise flow. An example of the thinking process using two velocity maps and RARE images as well as oscilloscope shots (Figure 4.10) will now be discussed:

- In the axial image the dot appears in the same location as in Figure 4.9 so the image is from the perspective of looking down at the geometry from above. Physically, the rotation of the inner wall is known to be clockwise (from motor flag). Red must then indicate flow toward the top of the velocity map and blue towards the bottom. On the scope images this colour direction choice correlates to the PGSE gradients stepping from negative to positive. The velocity encoding is in $y$, making $y$ the vertical axis that runs parallel to the planar regions length.

- For the planar slice image, the marker appears down from the top of the image so 'top' in the image map is also top in physical space. The marker is on the right so this slice is being viewed from an observer at the cylindrical region closest to the marker hole looking towards the planar region. Flow
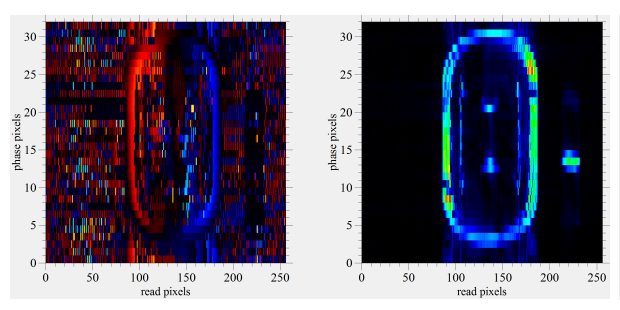

(a)

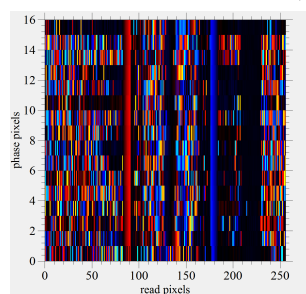

(d)

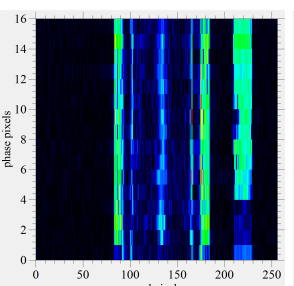

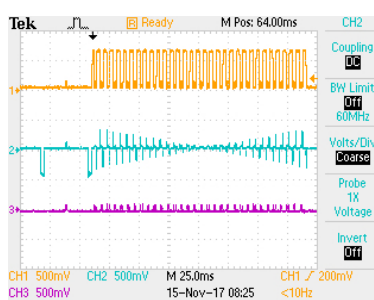

(b)

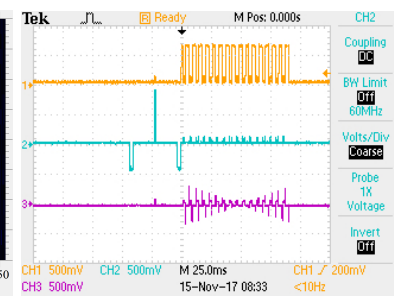

(e)

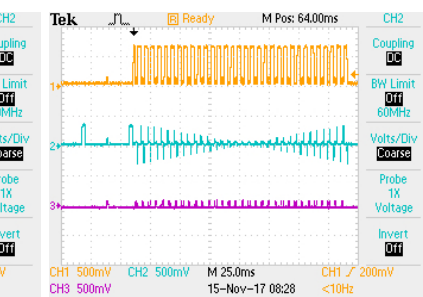

(c)

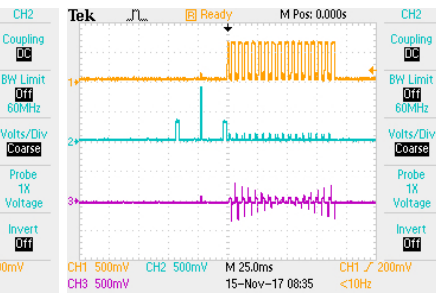

(f)

Figure 4.10: Orientation and flow experiment example for an axial (top row) and a offset planar (bottom row) slice. ( $a, d$ ) shows the velocity maps and corresponding RARE images positioned to pick up the marker hole (right on both images). (b, c, $\mathrm{e}, \mathrm{f})$ Are screen shots of the three gradient coil pulses sequences $(x, y, z$ from top to bottom) near the start (b,e) and end (c, f) of the NMR experiment. The q-gradients are the even height pair near the start of both $y$ lines. 
is still encoded along the $y$ axis stepping from negative to positive. Consequently the colours in this velocity map are assigned to exactly the same flow as in the axial slice. All these features are in agreement that the flow is into the page on the left and out of the page on the right.

Applying this logic to all the slices it is possible to construct an orientation and flow diagram, Figure 4.11. This also helps establish an understanding of how offsets defined in TopSpin translate to physical space in the geometry. The two possible $x y$ plane orientations shown are either defined by the signs of the offsets or the flow direction.

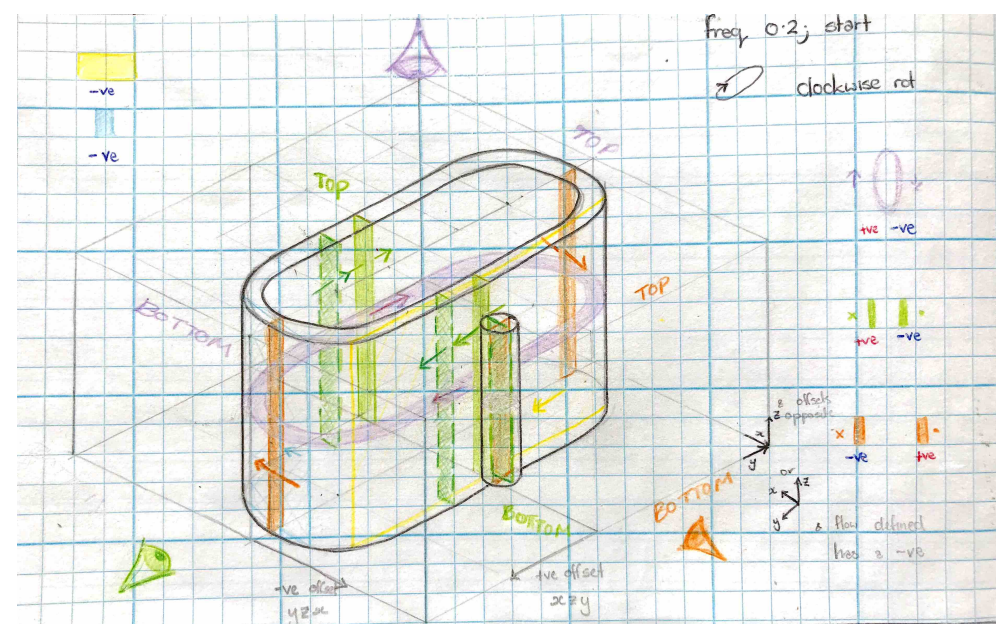

Figure 4.11: Orientation and flow of the three main slices: (purple) axial, (green) planar, and (orange) cylindrical. Arrows indicate flow direction, eyes show direction looking at the geometry for each slice (with top and bottom of each image labelled), and 2D pictures on right indicate colour and direction of flow for each raw $2 \mathrm{D}$ image taken.

Note: The choice of processing software is important as both TopSpin, Prospa and the Python script give different viewing perspectives as they process the data in slightly different ways. As Prospa is the most commonly used processing tool by this lab for quick, easily interactive analysis this flow and orientation study is done in terms on this. However a simple conversion between them can be made using the location of the marker in the images. Most result figures are done using Python script and so a conversion process was used to understand the figures. 


\title{
Chapter 5
}

\section{Shear Studies using PCH Geometry}

\begin{abstract}
A major aim of this work is to produce a new deformation environment with which to study complex materials. The PCH shear geometry and corresponding methodology that has been developed in this thesis, along with the pre-existing NMR techniques described in Chapters 2 and 3, has potential to achieve this and allows for a previously unstudied flow regime for rheo-NMR to be observed. This chapter presents the results of shear studies using both the (complex) shear banding WLM solution and the (simple) doped water. Both the planar and cylindrical regions are explored, with comparisons to velocity profiles of a TC shear geometry. Additional observations which pose open questions concerning interesting features and flows conclude the chapter.
\end{abstract}

\subsection{Observed Planar Region Flow Patterns}

The novel component of the geometry is that it combines closed streamlines with planar regions of flow. Hence it is the planar region which is of extra significance to explore. The two questions: 'how does the complex shear banding material behave?' and 'does the geometry produce the idealised planar Couette flow for simple fluid?' will be addressed.

\subsubsection{Evolution of Flow}

A powerful way to both qualitatively and quantitatively observe the evolution of a flow pattern is with the $2 \mathrm{D}$ axial slice velocimetry image described in Section 3.2.1 pages $46-47$. The same approach as Figure 3.9 is used to isolate the planar 
region, with a pixel resolution of $50 \mu \mathrm{m}$ across the width of the sample gap and $800 \mu \mathrm{m}$ along the planar region. To investigate the planar region the analysis was focused on an area the width of the gap and 17 pixels in length. From the actual pixel resolution the physical length of the planar region would be 16.25 however exactly centering this is difficult. As such the rows second in from either end are used as inclusive boundaries for the area of interest.

Tentative results for mapping the WLM flow pattern in this way suggest the following: the complex shear banding material studied does not, in fact, appear to shear band in the planar region. This can be seen in the Figure 5.1a for shear rates of $\dot{\gamma}=5 / \mathrm{s}$ and $\dot{\gamma}=10 / \mathrm{s}$ respectively (direction of flow is downwards). Both of these shear rates lie in the stress plateau region observed in the rheometer data, see Figure 3.3. Rows of data for the boundary and center of planar regions are extracted and plotted quantitatively in far right column of Figure 5.1a. They reveal a linear velocity profile throughout, with potentially an evolving offset as it progresses down the planar region (visually more apparent for the lower shear rate 2D image). The findings are tentative as the WLM sample presented some difficulties being imaged in this way ${ }^{1}$. However the rows in the maps which did not contain artifacts were still representative off all data seen to date. Consequently it appears that in the planar region the WLM sample's behaviour is more like that which would be expected for a Newtonian fluid under planar Couette flow.

The second of the two questions can be explored by shearing a Newtonian fluid, doped water, in the geometry. Identical tests to those conducted with the WLM were done for water and are displayed in Figure 5.1b. What is intriguing about these results is that while linear velocity profiles appear at the boundary of the planar region, the profile becomes curved in the center of the planar region, an evolution of the shape of the flow profile. This was reinforced by 1D velocimetry double slice selection tests with $\dot{\gamma}=5 /$ s spanning the planar region, as shown in Figure 5.2. It can be recalled that the most intense lines in this style of plot is the mean velocity, which helps to indicate the shape of the velocity profile. Both ends show the linear profiles, while in-between the ends a curved velocity profile shape develops asymmetrically in space.

A consideration for this could be that the deformation environment has transitioned into turbulence. The Reynolds number is a dimensionless variable that helps quantify the transition between laminar and turbulent flow for a fluid. Specifically it is the ratio of inertial and viscous forces, and written for Newtonian fluids in

\footnotetext{
${ }^{1} \mathrm{~A}$ few experiments had issues with optimising timing and gradient values and dealing with odd imaging features and artifacts.
} 

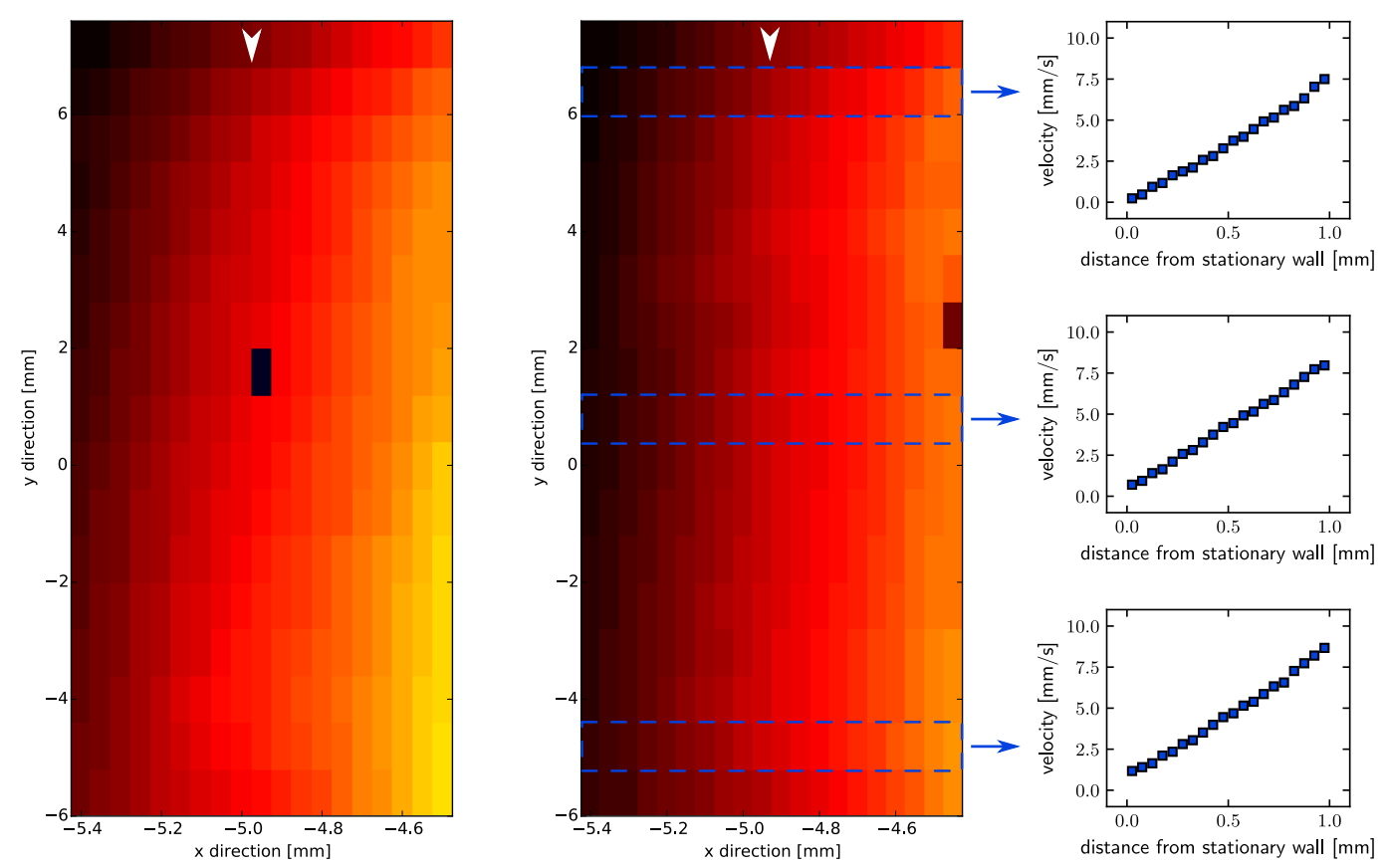

(a)
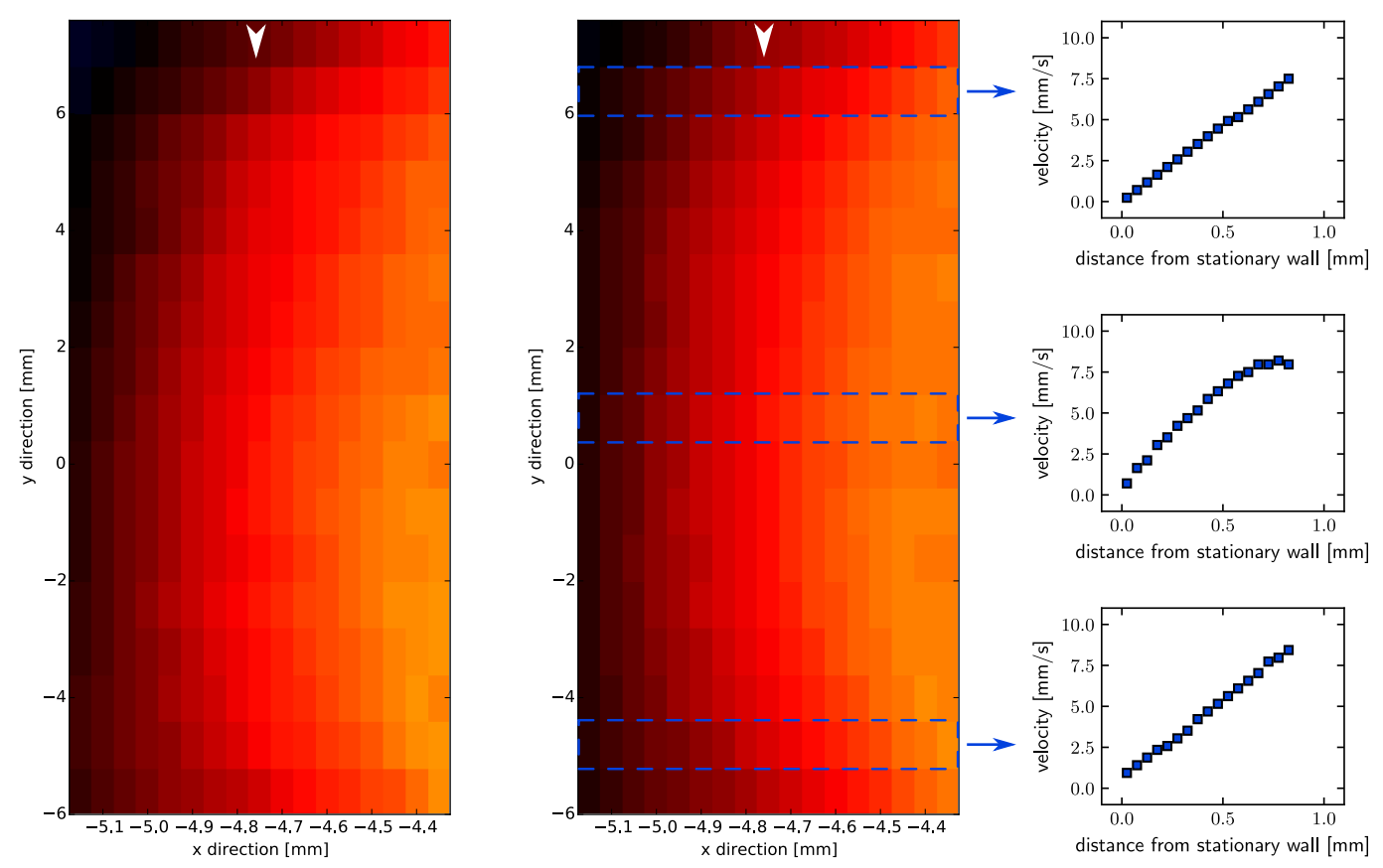

(b)

Figure 5.1: 2D axial velocity maps of planar region for $\mathrm{PCH}$ (physical area is approximately $1 \times 13 \mathrm{~mm}$ but has been stretched here to show flow pattern clearer). (a) is WLM solution, (b) doped water. From left to right: $\dot{\gamma}=5 / \mathrm{s}, \dot{\gamma}=10$ /s, velocity profiles across the sample gap taken from rows marked with a box. Direction of flow is downwards. 

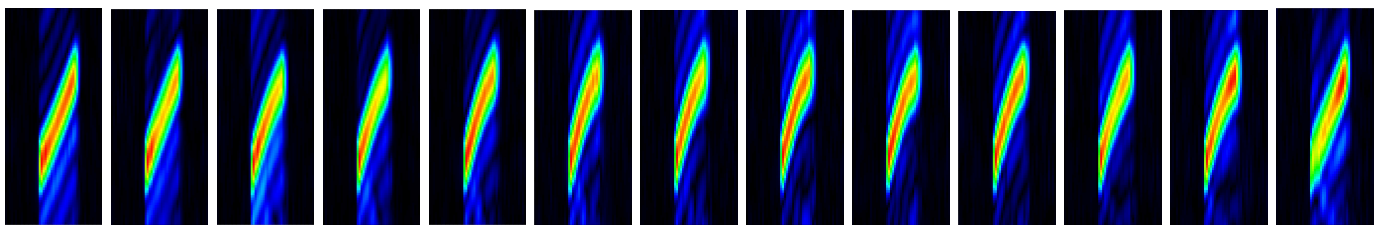

Figure 5.2: 2D displayed velocity distribution of water in PCH geometry sheared at $\dot{\gamma}=5 /$ s. Left to right shows the evolution of the shape of the velocity profile along the planar region taken at $1 \mathrm{~mm}$ intervals.

planar shear is

$$
R e=\frac{\rho v d}{4 \eta}
$$

where $v / 2$ is the characteristic velocity ( $v$ is the velocity of a single moving wall) and $d / 2$ is the characteristic wall separation (half the actual plate separation $d$ ) [64]. In Krug et al. [55], a study of the fluid dynamics planar cylindrical geometries, it was shown that a Reynolds number $<320$ indicates that the materials is in the laminar flow regime. For water at room temperature and a wall separation of $1 \mathrm{~mm}$ equation (5.1) simplifies to approximately the velocity (in units of $\mathrm{mm} / \mathrm{s}$ ) over 4 . The dimensionality of the PCH conveniently makes velocity and shear rate roughly equal (just with a change of units). This means that for the shear rates explored in this thesis the Reynolds number suggests that the flow is still in the laminar regime.

As seen in Figure 5.1b, the water is not demonstrating planar Couette flow. However this exciting flow pattern could imply a complicated deformation environment that is imparted by the geometry. This deformation may in turn be responsible for the 'simple' behaviour of the complex material. It can be concluded that as the WLM solution acts differently to water it is still responding to the deformation in a complex way, however, it manifests itself as a deceptively simple flow.

What is visible in Figure 5.1 is how the asymmetry along the direction of flow in the velocity profiles is more apparent for the lower shear rate example for both samples. However, extracted rows of $1 \mathrm{D}$ data from the 2D velocity maps did still show the same overall evolution, or lack thereof, for both shear rates.

\subsubsection{Function of Shear Rate}

Other tests were conducted solely focusing on a PGSE-RARE $1 \mathrm{~mm}$ planar slice, which was centered on the PCH's planar region. From these experiments $1 \mathrm{D}$ velocity profiles were extracted and plotted in the form of Figure 3.12 using the methods discussed in Section 3.2. The planar slice experiment allowed for a large range of shear rates to be explored as part of an effort to gauge the influence of shear rate on 
the shape of the flow profile.

Image resolutions for these experiment are shown in Table 5.1. These also include details pertaining to the TC benchmark comparisons discussed in the next section. From the table, the number of sample points across the gap for these resolutions is approximately $25-26$ for a nominal $1 \mathrm{~mm}$ sample gap ${ }^{2}$. The actual number of points used to resolve the velocity profile is not fixed and so may vary in the later plots. As the number of sample points is influenced by the actual dimensionality of the device and residual mechanical influences mentioned in the previous chapter, the RARE image is used to determine the number of sample points.

\begin{tabular}{c||c|c|c}
$\begin{array}{r}\text { Properties } \\
\text { of Images }\end{array}$ & $\begin{array}{c}\text { Effective } \\
\text { FOV [mm] }\end{array}$ & Pixels & $\begin{array}{c}\text { Voxel Size for } \\
\text { 1D Plots [mm] }\end{array}$ \\
$\begin{array}{c}\text { Geometry } \\
\text { and Sample }\end{array}$ & & & \\
\hline \hline PCH - WLM & $20 \times 10 \times 1$ & $512 \times 8 \times 1$ & $0.039 \times 10 \times 1$ \\
PCH - Water & $20 \times 10 \times 1$ & $512 \times 8 \times 1$ & $0.039 \times 10 \times 1$ \\
TC - WLM & $30 \times 10 \times 1$ & $512 \times 4 \times 1$ & $0.059 \times 10 \times 1$ \\
TC - Water & $20 \times 10 \times 1$ & $512 \times 8 \times 1$ & $0.039 \times 10 \times 1$
\end{tabular}

Table 5.1: Imaging resolutions for the 1D velocity plots collapsed down from 2D velocity map data. The FOV in phase was actually $20 \mathrm{~mm}$ with only the center $10 \mathrm{~mm}$ averaged over. Note that third row is data collected and included with permission by Tim Brox and used a 1D velocimetry experiment instead.

Dependency on shear rate in the PCH geometry for both samples can be seen in Figure 5.3. The range of shear rates that are presented are restricted by a maximum shear of $\approx 20 / \mathrm{s}$. This makes the information more meaningful as it aligns with the expected range for observing the WLM shear banding effect. Visually the shapes of the WLM and water profiles here are consistent with the shapes observed in the previously discussed experiments. For the WLM sample, shear banding was not evident at any of the shear rates. For water, the curved profile appears to be present throughout the shear rate range. Potential implications of this are that, while there exist asymmetry in the flow pattern along the planar region, at no point of the range tested does this alter enough to change the qualitative shape of the velocity profile in the center of the planar region in the $\mathrm{PCH}$ geometry.

\footnotetext{
${ }^{2}$ With the exception of the TC - WLM data which would be 17 .
} 


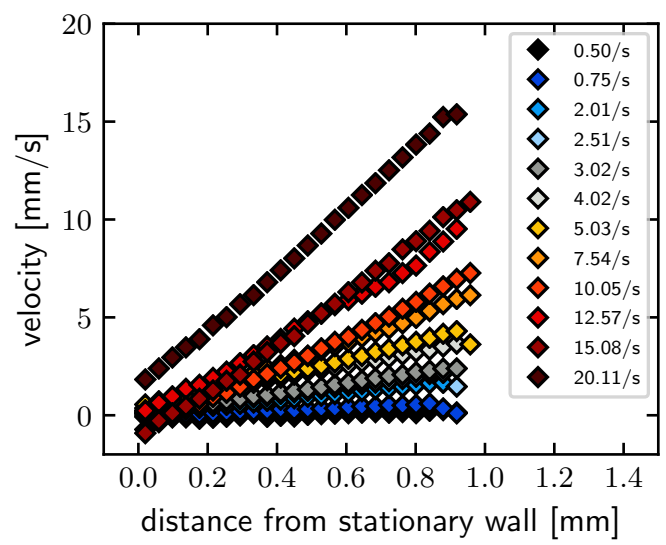

(a)

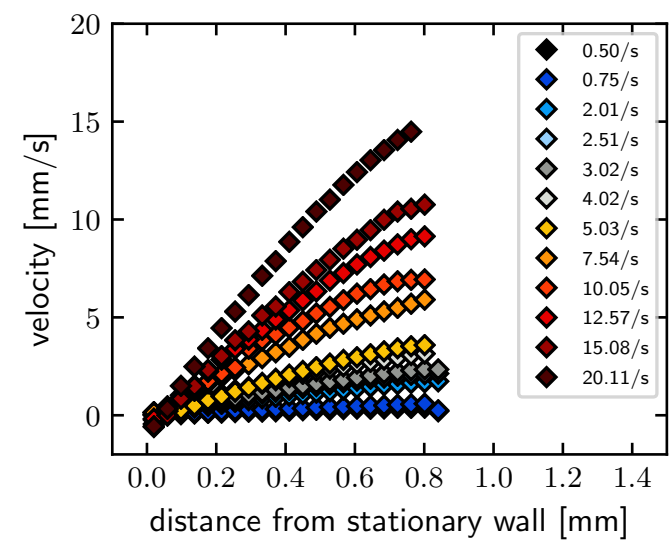

(b)

Figure 5.3: Velocity profiles across the gap in the $\mathrm{PCH}$ geometry for a range of shear rates with (a) WLM (b) doped water. Profiles come from planar slices taken at the center of the planar region. Imaging artifacts were more prevalent in the velocity profiles of WLM at lower shear rates, consequently corresponding shear rates have been removed from water plots.

\subsubsection{Comparison to Standard Geometries}

The Taylor-Couette shear geometry can act as a benchmark for the PCH tests because it is one of the most common rheo-NMR geometries and has been well explored in terms of its impact on simple fluids and shear banding materials. The device specifically used here was shown in Figure 2.14a, and like those previously used in NMR WLM research $[3,4,48,60]$, has $r_{i}=8 \mathrm{~mm}$ and $r_{o}=9 \mathrm{~mm}$.

To compare the TC to the novel geometry the same method was used with PGSE-RARE to collect 1D velocity information across the gap. The imaging resolution was kept the same, see Table 5.1, except for the data collected for the TC geometry studying the WLM sample. It suffered from misalignment of the inner cylinder and from image artifacts. However the shear banding phenomena has been well studied for the CPCl NaSal WLM system therefore data presented here in Figure 5.4a were collect by Tim Brox [4] and used with his permission. It is same WLM (with different $T_{1}$ reducing agent) in the same TC geometry. Figure 5.4b displays the water data.

It is clear from these plots the contrast in both the simple and complex material behaviours for the common Taylor-Couette designs and this novel shear geometry, even though they are both aiming to approach the same homogeneous flow con- 


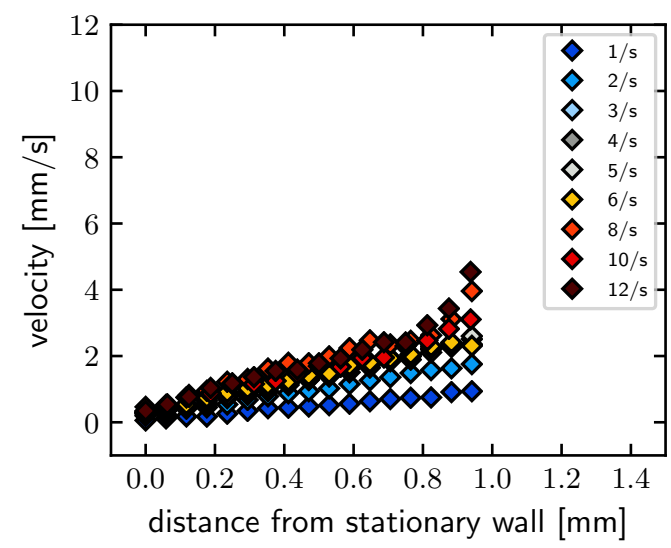

(a)

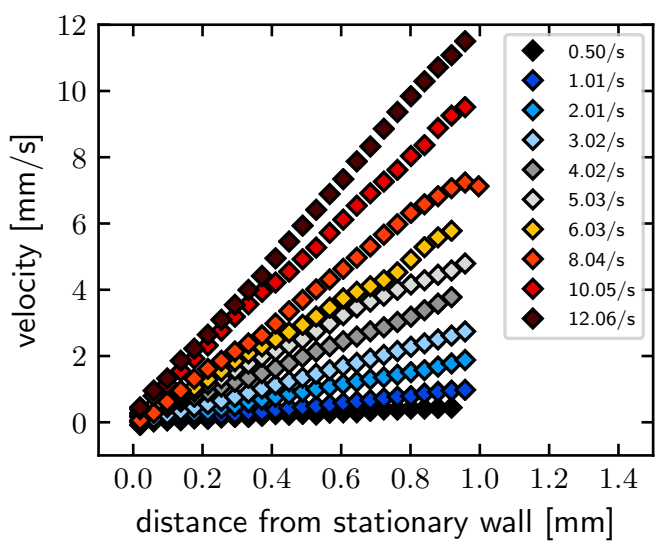

(b)

Figure 5.4: Velocity profiles across the gap in the TC geometry for a range of shear rates with (a) WLM (b) doped water. (a) is used with permission from T.I. Brox. These are done with geometries with identical radii. The higher shear rate band is starting to become visible at the higher shear rates presented. The other stark indication between the two behaviours is that when the material is shear banding the lower shear band rate is approximately constant and the velocity profiles stack up.

dition. What is intriguing is WLM sample exhibits shear banding both in the TC geometry and (evident in rheological data) in more ideal cone and plate geometry. For simple fluids these geometries approximate homogeneous deformation. The doped water evolution of the flow profile in the $\mathrm{PCH}$ geometry shows that simple homogeneous planar flow is not occurring, suggesting that the geometry imparts some sort of non-uniform deformation. However it is under these deformation conditions where the WLM behaves very differently. This makes the PCH geometry truly novel in the dynamics it induces in well explored samples. This could open the way to different applications of WLM materials.

\subsection{Observed Cylindrical Region Flow}

As discussed above, indication of shear banding for the WLM solution is not apparent in the planar region experiments. The other geometric component of the $\mathrm{PCH}$ geometry is the cylindrical regions, similar to two halves of a TC geometry. Hence, though not the main area of interest for the novel geometry, it is meaningful to briefly investigate if shear banding arises in the more familiar shaped region. 

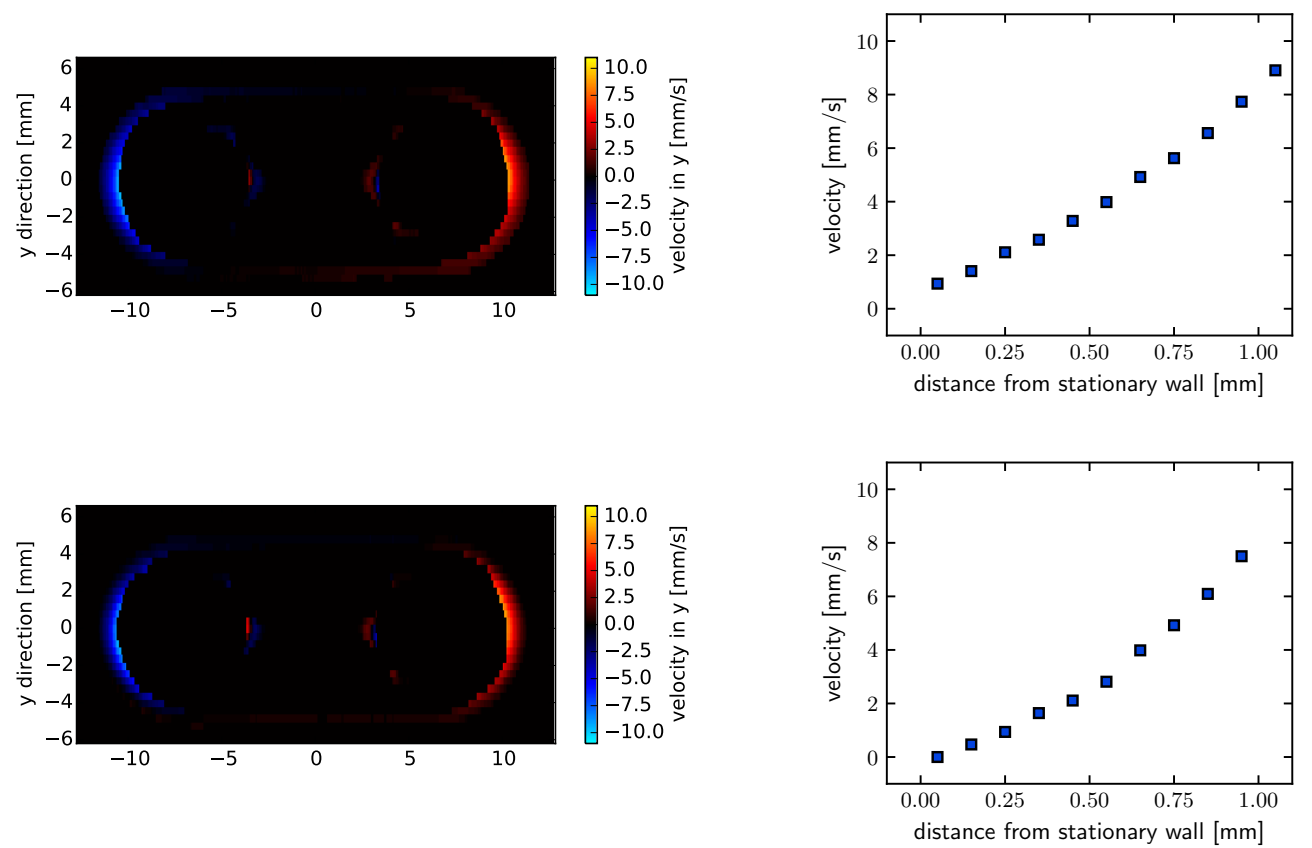

Figure 5.5: 2D velocimetry maps studying flow in cylindrical regions at a shear rate of $\dot{\gamma}=10$ /s with (top row) WLM and (bottom row) doped water in PCH shear geometry. Velocity is encoded in vertical direction. Extracted velocity profiles in the center of the cylindrical region for the left gap are positioned directly to the right of the maps. Flow is the geometry is clockwise.

The same method for Section 5.1.1 can be used, but instead with the velocity encoded parallel to the flow direction in the center of the cylindrical regions. The center row (at $y \approx 0$ for velocity maps in Figure 5.5) can be extracted and plotted to reveal the shape. Here in Figure 5.5 the entire geometry is imaged, but the cylindrical regions are more easily visible as flow in the planar regions now run mostly perpendicular to the velocity encoding direction. The FOV was $25.6 \times 12.8 \mathrm{~m}$ with $256 \times 32$ pixels, giving a $100 \mu \mathrm{m}$ pixel resolution spanning the cylindrical gap. A step down in resolution from the planar region 2D studies was necessary to provide sufficient signal-to-noise and reduce image flow distortions (see Section 3.3.4).

What it exciting to observe is that the complex material does not appear to shear band in the cylindrical regions either, instead a Newtonian-like, slightly curved, profile is shown (a visible curved profile is more expected for a Newtonian fluid in this geometry's cylindrical region as it has a less ideal $\kappa$ value of 0.8$)$. In this instance water shows a very similar response to that expected, though there is insufficient resolution to resolve the bend in the curve. Any complex manifestations 
in the WLM case to distinguish it from the Newtonian deformation are subtle.

For the WLM solution the curved profile shapes appears to scale with shear rate as depicted in Figure 5.6. This was studied at a similar shear rate range to the planar region work. The different behaviour of the WLM solution in the PCH geometry continues as it does not shear band even in the curved regions (similar to sections of a TC geometry) at all applied shear rates, only to have an increased data noise.

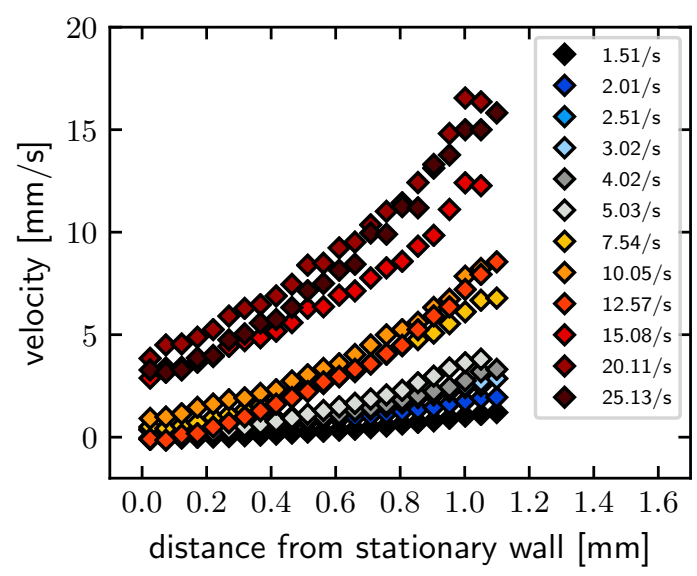

Figure 5.6: Velocity profile across the cylindrical gap in the $\mathrm{PCH}$ geometry for a range of shear rates with WLM. Obtained from a 2D PGSE-RARE $1 \mathrm{~mm}$ cylindrical slice collapsed down to 1D.

\subsection{Additional Observations}

Throughout the shear studies conducted on the PCH geometry additional effects of the flow behaviour, sample performance, and mechanical influences were observed. They will each be briefly discussed in this sections with some contemplation on their impact with regards to the main study.

\subsubsection{Degrading WLM Sample}

Changes in velocity profiles and $T_{2}$ characteristics of a WLM sample over time were observed while the material was loaded in the PCH geometry.

The first loading of the WLM solution was not subjected to its velocimetry experiments until almost a fortnight after it was initially put into the geometry, and had spent approximately $31 / 2$ days and one overnight in the magnet prior to this 
(where is it not continuously kept at or above $25^{\circ} \mathrm{C}$ nor Parafilmed to be kept more air tight). At this stage $T_{2} \approx 160 \mathrm{~ms}$, which is significantly lower than two later trials where the sample was loaded and tested with velocimetry experiments on consecutive days ( $T_{2} \approx 210,220 \mathrm{~ms}$ ). These experiments were also in better agreement with the relaxometry WLM tests conducted separately with sample in a NMR tube ( $T_{2} \approx 230 \mathrm{~ms}$ ). One of the two later PCH loaded samples was monitored for 5 days (with under 3 days magnet time) and over that period of time showed a decrease in $T_{2}$ of $10 \%$.

Further to that, the velocimetry results for the first loading otherwise conducted identically to those displayed for a later loading in Figure 5.3a showed bent velocity profiles. This shape, shown in Figure 5.7, neither directly resembles the planar profile shown in the 2D planar region maps or the 2D to $1 \mathrm{D}$ center of planar region slices tests for the other WLM experiments, nor any tests with doped water. From

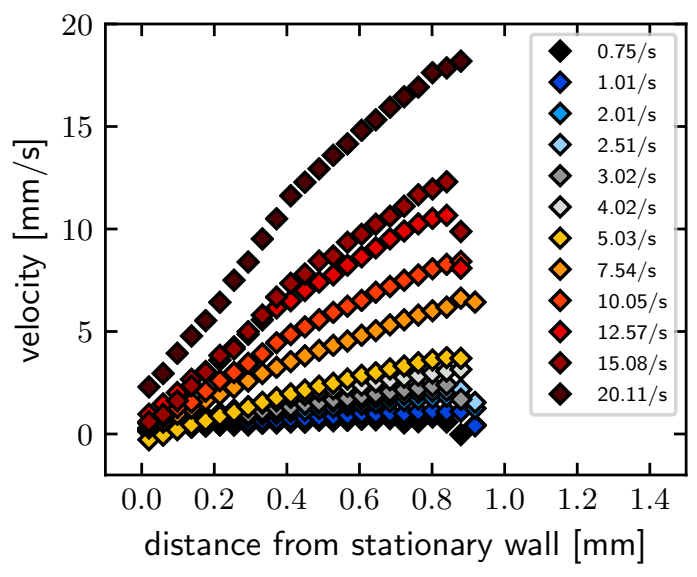

Figure 5.7: Velocity profiles across the planar region gap in the $\mathrm{PCH}$ geometry for a range of shear rates with degraded WLM. Planar slice was taken at the center of the planar region.

this the importance of timing was considered in later experiments with this sample, however, as it was not a main focus of this research its source was not further tested for. A tentative suggestion for this degrading of the WLM sample is the exposure to air, as the geometry can not be Parafilmed while it is being turned inside the magnet system. This might change the concentrations and potentially the structure of the solution as it dries out. For further exploration, reactions with the geometry / band material and the Vaseline could additionally be considered. 


\subsubsection{Variation in Gap}

The nominal geometry dimensions are shown in the lower portion of Figure 4.1, where the band and gap thickness both measure $1 \mathrm{~mm}$. ParaVision MRIs can be used to get a handle on the physical dimensions. For planar slice experiments an equivalent MRI was taken averaging over the centre $10 \mathrm{~mm}$. From this data, the band thickness was found to be $0.96 \mathrm{~mm}$ and $0.98 \mathrm{~mm}$ (WLM tests, $\mathrm{H} 2 \mathrm{O}$ tests) and the gap $0.91 \mathrm{~mm}$ and $0.87 \mathrm{~mm}$ (WLM tests, $\mathrm{H} 2 \mathrm{O}$ tests) in the center of the planar region with an error of $\pm 6 \%$. The specific band used and how it is placed on the rotors may effect this.

A slight variation over the length of the planar region is revealed in the MRI shown in Figure 5.8, where the boundaries of the planar region have an additional width of $0.150(+0.025-0.050) \mathrm{mm}$ compared to the center. This thickness is conserved (within $0.025 \mathrm{~mm}$ ) around the cylindrical regions. The variation is at the inner wall, reinforcing the method of using the fixed point for plotting comparison tests as the outer wall. The construction limitations in executing the design along

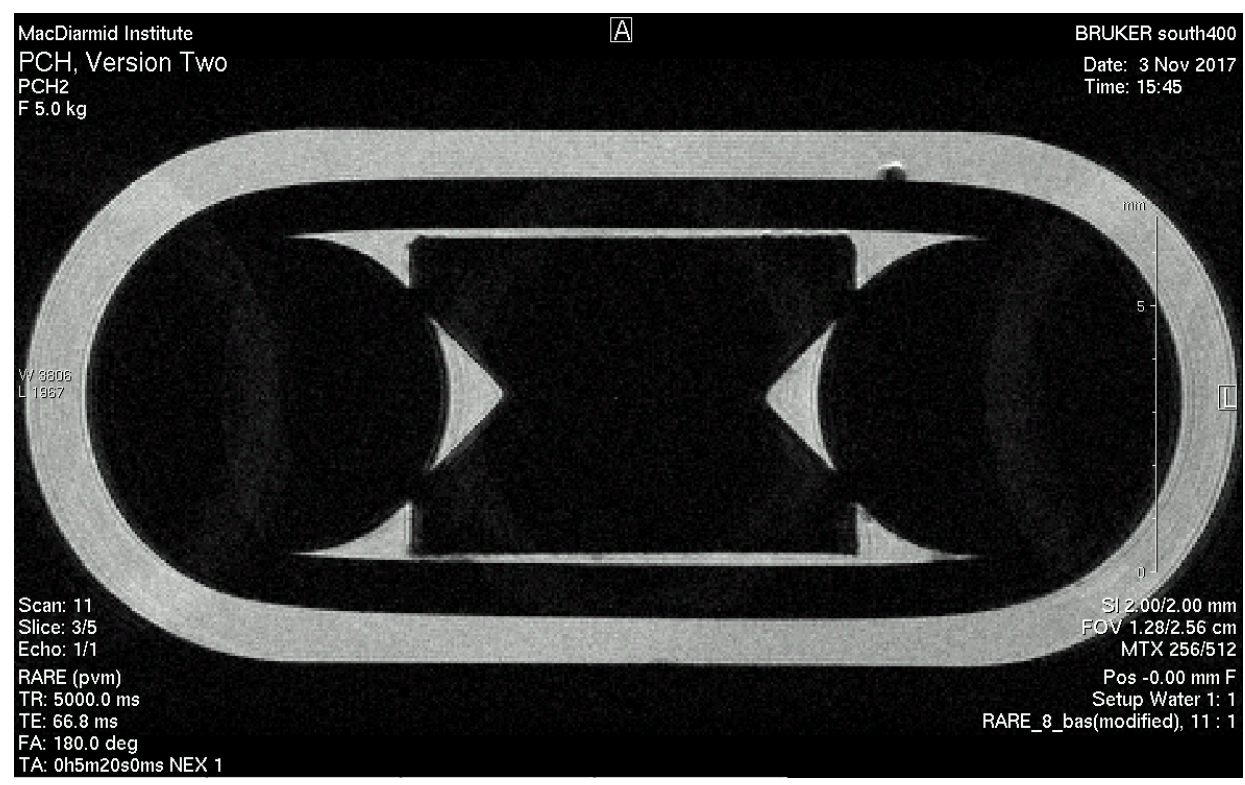

Figure 5.8: ParaVision MRI showing variation in gap thickness.

with the apparent bowing of the band in the center of the planar region, impacts the deformation that the device generates (which can be observed) and potentially the shear rate. In particular, as the velocity profiles obtained through this research do not exhibit shear rate dependant features, an uncertainty of the exact shear rate is not of major concern. However a variation in shear rate around the $\mathrm{PCH}$ geometry may be an influencing factor in the behaviour shown in previous tests. 
All shear rates stated so far in this thesis have been based off the assumption that the band is a rigid body around the rotors, as well as of the nominal dimensions. Based off equation (2.8) for the TC geometry where the shear rate is the inner wall velocity over the gap gives

$$
\dot{\gamma}(r)=\frac{2 \pi f r_{i}}{r_{o}-r_{i}}=\frac{2 \pi f(3+1) m m}{1 m m}=8 \pi f
$$

where $f$ is the rotation frequency of the rotors with is in direct correspondence with the motor frequency. If, instead, the dimensions determined from the MRI are used then this $8 \pi$ proportionality between shear rate and frequency increases (by less than $20 \%$ ) in the planar region and decreases (by less than 15\%) at the boundaries and in the cylindrical region (due to the variation in gap). If the assumption of the rigidity of the band is allowed to be relaxed both gap variation and band deformation are in play in deforming the inner wall speed in the geometry. This opens up the question of the actual applied shear rate, which could motivate additional study, and is explained in more detail in Chapter 6.

\subsubsection{Secondary Flow}

Secondary flow is any component of flow which is travelling in an orthogonal direction to the dominant flow direction. In the $\mathrm{PCH}$ geometry the dominant flow direction is parallel racetrack shape. Considering the velocity vector for an element of material under flow, secondary flow is any component of the vector either along $z$ or pointing perpendicular to the outer wall of the racetrack. As the $\mathrm{PCH}$ geometry does not exhibit planar Couette deformation but a more complex pattern and there appears to be variation in gap thickness along the planar region, it was of interest to consider the extent of secondary flows in the system.

An example of secondary flow can be seen in the axial velocity maps in Figure 5.5. In these experiments the velocity was encoded in the $y$ direction with both of the samples moving clockwise around the geometry. While faint compared to the cylindrical region flow observed, it is possible to see a $y$ direction velocity signal in the planar regions of these maps. The most notable secondary flow occurs at either boundary between the planar and cylindrical region, in particular at the end where the sample has just left the cylindrical region. Specifically the plots reveal that the sample is being constricted by the inner wall at the point of entry into the planar region. In exiting the planar region the sample, much less significantly, moves towards the inner wall. This could be consistent with a band that is slightly bowing in the planar region leaving thicker sample gaps in which the fluid can 
flow at the boundaries. Secondary flow in the planar region sample gap in the $z$ direction was imaged and found to be negligible.

\subsubsection{Backflow and Offset}

One PGSE-RARE 2D axial velocity map of the PCH geometry with WLM exhibited significant backflow at the stationary wall (see Figure 5.9). It is of interest to note as this was not apparent at higher or lower shear rates or in the reverse flow direction. Infrequently this would also be seen in planar slice 1D velocity data too. However this was not always repeatable. Whether this is actual flow and how this is generated in this instance and not others could be resolved with mathematical simulation of the sheared flow for this geometry (which was not incorporated in the scope of this work).

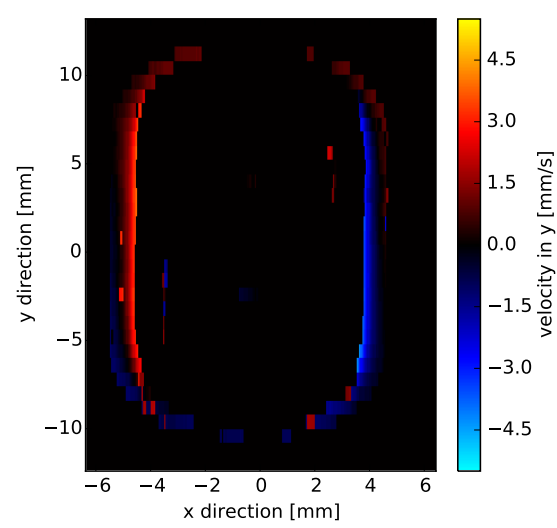

(a)

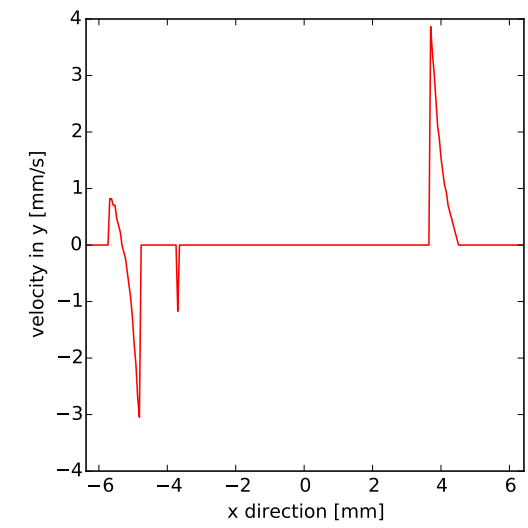

(b)

Figure 5.9: Example backflow in the $\mathrm{PCH}$ geometry in a 2D axial image (not to proportion) with velocity encoded in the vertical direction, with flow $\dot{\gamma}=5 / \mathrm{s}$. 1D velocity information extracted at $\approx-4 \mathrm{~mm}$.

For some 1D data the negative flow at the stationary wall could instead be due to an offset in the processed velocities. For this research the shape rather than explicit values of the flow profile is of greater focus. However this is something to be considered for further development of NMR procedures and potentially refining pulse sequences. 


\section{Chapter 6}

\section{Future Work}

Building upon the work presented in this thesis, here is a brief discussion of possible future directions for the planar cylindrical hybrid shear geometry development and use.

\section{Mathematical Modelling}

A future step for understanding the dynamics of this novel geometry is to mathematically model its flow for a simple fluid. This analysis could provide conformation for the dominant flow pattern and hopefully contain suggestions for the origin of additional flows seen. Differences may give a better indication between flow artifacts that result from the specific NMR experimental conditions and the nonhomogeneous nature of the flow pattern. Talks have already started with potential colleagues who are specialised in these techniques.

\section{Actual Applied Shear Rate}

The actual applied shear rate by the band is not something that can be simply assumed for the complete characterisation of the novel geometry's deformation environment. The rheo-NMR device is on the dimensions where the finite thickness of the band and the deformation of the band itself could be important. This stems from the fact that the mould for the band is a cylinder shape and is stretched into the racetrack formation by the two rotors (resulting in areas of slightly lower and higher curvature). Physically the outer surface of the band, which is in contact with the sample, is being stretched in the cylindrical regions and contracted in the planar regions. For a constant rotation rate of the rotors this could produce slightly 
different inner wall velocities, and hence shear rates, depending on the location in the racetrack.

Current NMR velocimetry techniques cannot be used to 'see' the deformation and dynamics of the band as it was deliberately chosen to have a negligible NMR signal so only the actual rheological sample would show up in images. To investigate the actual applied shear at the inner wall objects could be attached to the band which would create imaging artifacts in MRIs.

\section{Dimensionality and Aspect Ratio}

The specific dimensionality of the design impacts sample behaviour as shown in previously cited work and in this thesis.

For pipe flow it is known that a certain aspect ratio (length of pipe to internal diameter) is required for flow to stabilise. As evolution of flow is apparent in the $\mathrm{PCH}$ geometry, further lengthening of the planar region, with respect to its width, may allow for more homogeneous flow to develop. On a grand scale, the geometry could be enlarged by several orders of magnitude to instead loop through and around a horizontal MRI system with only one planar region situated inside the magnet. On a more subdued scale, a slight enhancement could come from a thinner band and a narrower racetrack hole. What could become significant to study in this case is effects from in and out flow of the increased curvature in the cylindrical regions.

A thinner band and tighter alignment holes for the top ends of the rotors (negating the need for an insert) may improve the uniformity of the gap thickness and the potential slight variation of the applied shear rate in different regions. This would work further towards homogeneous deformation but could be at a loss of band integrity.

\section{Adapting for Torque and Oscillatory Experiments}

The PCH device presents a novel flow environment with which to study complex materials, already with interesting new responses from a shear banding sample.

Another experimental technique that can be coupled to further extend the testing options is trialling an oscillatory capable rheo-NMR drive shaft. This has the potential to trap the sample in the planar region and study more of the elastic behaviour of materials.

Adaptions could also include working with a torque sensor drive shaft to aim 
for simultaneous torque and NMR experiments. This would require a mechanical analysis and future development of the device, similar to that shown for the TC geometry, to relate torque to shear stress.

\section{Wormlike Micelles at Higher Shears}

On the study of a complex shear banding material, the WLM solution did not visibly show a banded structure in the novel geometry. Higher shear rates did experience a deviation from the expected value applied by the wall. Higher resolution NMR imaging and developing NMR experimental conditions to better handle faster flows could be developed that attempt to understand the behaviour observed in this work, including the results of even higher shear rates. 


\section{Chapter 7}

\section{Conclusions}

\subsection{Summary of Presented Work}

This thesis presents work aimed at achieving a novel deformation environment to study complex materials for rheo-NMR. To this end, the development of a novel planar cylindrical hybrid geometry is communicated. This development comprises both the redesign of the original novel geometry (which precedes this work) and operating procedures. Shear NMR studies with the geometry are found to be achievable and results further highlight the significance of deformation environment on a material's response.

Firstly, the issues in the original version of the geometry, mainly to do with alignment of the flow environment, were addressed. The vertical straightness and positioning of the shear rotors was improved by separating the cup piece of the original geometry into a tube and plug, making it easier to precisely machine. Enhancing the alignment of the flow environment as well as the geometries positioning with imaging system was further done by adapting how the three components of the geometry and the RF coil were assembled. Band construction was also slightly refined.

In conjunction with the new device, and its greater number of individual components, a specific assembly procedure was outlined in this thesis. Additionally included was a set of pre-experiment checks using MRIs to ensure proper set up and sample conditions. For the NMR system itself a Meech Coldstream Air Gun was added to the pre-existing temperature control system to provide more ideal conditions for maintaining a fixed sample temperature just below / around ambient. Two one-off tests were conducted as part of pre-experiment set up, an imaging system calibration as well as experiments to relate the orientation and flow of the physical system to the processed NMR experiments. 
In terms of shear studies the main appeal of the planar cylindrical hybrid design was the planar regions with continuous flow. Focusing on this area with the aid of spatially encoded velocimetry pulse sequences the velocity profiles of both a complex shear banding WLM material and a simple Newtonian fluid were obtained. Though the WLM material undergoes shear banding in most common rheometry geometries, the phenomena was not visually present in the novel geometry, instead a Newtonian-like linear profile was observed. Further exploration led to the conclusion that this was still a complex behaviour as it did not match the curved profiles seen for the simple fluid whose shape also evolved over the length of the planar region. A brief study of the cylindrical region did not show evidence of the shear banding either. The disappearing of such a well documented phenomena in the novel geometry is both exciting and intriguing. It invites further questions around the influences of the shear banding behaviour of WLM as well as the effect of different deformation environments on material behaviour in general.

The planar evolution results presented here are tentative with the desire to further optimise the methodology to remove NMR imaging features and ensure the ability to investigate a greater variation of the shear rates with axial maps. Further observations like gap variation, secondary flow, and backflow are commented on along with the potential for further investigation.

\subsection{Final Remarks}

This work on the Planar Cylindrical Hybrid shear geometry was conducted as part of the continued enrichment of rheo-NMR by the Magnetic Resonance Physics Group at Victoria University of Wellington and prior to that at Massey University. For the past four decades the group has endeavoured to push boundaries, applying the full NMR toolbox (including and beyond the standard NMR techniques of spectroscopy and imaging), to study nature. Research has been motivated to understand soft matter, including those of rheological interest, and investigate and improve deformation tools used. This incorporates both methodology (i.e. pulse sequence) and hardware (both in-house and commercial) development. The $\mathrm{PCH}$

geometry presents a new device with which to continue this investigative journey, whether it be in the current form presented here or with adaptions to more advanced drive shafts or physically on grander scales. 


\section{Bibliography}

[1] J. F. Steffe, Rheological Methods in Food Process Engineering. Freeman Press, 1996.

[2] S. Manneville, "Recent experimental probes of shear banding," Rheologica Acta, vol. 47, no. 3, pp. 301-318, Apr 2008.

[3] T. I. Brox, B. Douglass, P. Galvosas, and J. R. Brown, “Observations of the influence of taylor-couette geometry on the onset of shear-banding in surfactant wormlike micelles," Journal of Rheology, vol. 60, no. 5, pp. 973-982, 2016.

[4] T. I. Brox, "New methods for studying materials under shear with nuclear magnetic resonance," phdthesis, Victoria University of Wellington, 2016.

[5] The Society of Rheology, "Editorial," Journal of Rheology, vol. 1, no. 1, pp. 9395, 1929.

[6] A. S. Hunter, "Minutes of the meeting of the committee on organization of the society of rheology held at columbus, ohio, on april 29, 1929," Journal of Rheology, vol. 1, no. 1, pp. 95-97, 1929.

[7] C. W. Macosko, Rheology: Principles, Measurements, and Applications. Wiley$\mathrm{VCH}, 1994$.

[8] H. Barnes, J. Hutton, and K. Walters, An Introduction to Rheology, ser. Rheology Series. Elsevier, 1989, vol. 3.

[9] R. G. Larson, The Structure and Rheology of Complex Fluids, ser. Topics in Chemical Engineering. Oxford University Press, 1999.

[10] W. R. Schowalter, Mechanics of Non-Newtonian Fluids. Pergamon Press, 1978.

[11] M. Reiner, Deformation, Strain and Flow: An Elementary Introduction to Rheology, 2nd ed. H. K. Lewis and Co. Ltd., 1960. 
[12] K. Walters, Rheometry. Chapman and Hall Ltd., 1975.

[13] V. N. Constaninescu, Laminar Viscous Flow, ser. Mechanical Engineering Series. Springer New York, 1995.

[14] G. F. C. Searle, "A simple viscometer for very viscous liquids," in Proc. Cambridge Philos. Soc, 1912.

[15] M. M. Couette, "Studies relating to the motion of liquids," Ann. Chim. Phys, vol. 21, no. 6, pp. 433-510, 1890.

[16] M. Mooney and R. H. Ewart, "The conicylindrical viscometer," Physics, vol. 5, no. 11, pp. 350-354, 1934.

[17] D. V. Boger and K. Walters, Rheological Phenomena in Focus, ser. Rheology Series. Elsevier Science, 2012, vol. 4.

[18] J.-F. Berret, Rheology of Wormlike Micelles: Equilibrium Properties and Shear Banding Transitions. Dordrecht: Springer Netherlands, 2006, ch. 19, pp. 667-720.

[19] J. P. Rothstein, "Strong flows of viscoelastic wormlike micelle solutions," Rheology Reviews, vol. 2008, pp. 1-46, 2008.

[20] F. Bloch, “Nuclear induction," Phys. Rev., vol. 70, pp. 460-474, Oct 1946.

[21] E. M. Purcell, H. C. Torrey, and R. V. Pound, "Resonance absorption by nuclear magnetic moments in a solid," Physical review, vol. 69, no. 1-2, p. 37, 1946.

[22] P. T. Callaghan, Principles of Nuclear Magnetic Resonance Microscopy. Oxford University Press, 1991.

[23] A. Abragam, The Principles of Nuclear Magnetism, ser. The International Series of Monographs on Physics. Oxford University Press, 1973.

[24] C. P. Slichter, Principles of Magnetic Resonance. Springer Berlin Heidelberg, 1996.

[25] H. C. Torrey, "Bloch equations with diffusion terms," Phys. Rev., vol. 104, pp. 563-565, Nov 1956.

[26] S. Meiboom and D. Gill, "Modified spin-echo method for measuring nuclear relaxation times," Review of scientific instruments, vol. 29, no. 8, pp. 688-691, 1958. 
[27] P. T. Callaghan, Translational Dynamics and Magnetic Resonance: Principles of Pulse Gradient Spin Echo NMR. Oxford University Press, 2011.

[28] T. R. Saarinen and C. S. Johnson, "Imaging of transient magnetization gratings in nmr. analogies with laser-induced gratings and applications to diffusion and flow," Journal of Magnetic Resonance (1969), vol. 78, no. 2, pp. 257 - 270, 1988.

[29] T. Scheenen, D. van Dusschoten, P. de Jager, and H. V. As, "Microscopic displacement imaging with pulsed field gradient turbo spinecho nmr," Journal of Magnetic Resonance, vol. 142, no. 2, pp. 207 - 215, 2000.

[30] P. Galvosas and P. T. Callaghan, "Fast magnetic resonance imaging and velocimetry for liquids under high flow rates," Journal of Magnetic Resonance, vol. 181, no. 1, pp. $119-125,2006$.

[31] S. Kuczera and P. Galvosas, "Advances and artefact suppression in rarevelocimetry for flow with curved streamlines," Journal of magnetic resonance (San Diego, Calif. : 1997), vol. 259, p. 135145, October 2015.

[32] J. Hennig, A. Nauerth, and H. Friedburg, "Rare imaging: a fast imaging method for clinical mr," Magnetic resonance in medicine, vol. 3, no. 6, pp. 823833, 1986.

[33] S. A. Rogers and P. T. Callaghan, "Time-dependent nmr-velocimetry of a colloidal glass," Rheologica Acta, vol. 48, no. 7, pp. 735-745, Aug 2009.

[34] A. F. Martins, P. Esnault, and F. Volino, "Measurement of the viscoelastic coefficients of main-chain nematic polymers by an nmr technique," Phys. Rev. Lett., vol. 57, pp. 1745-1748, Oct 1986.

[35] M. Vera and J. B. Grutzner, "The taylor vortex: the measurement of viscosity in nmr samples," Journal of the American Chemical Society, vol. 108, no. 6, pp. 1304-1306, 1986.

[36] S. Lacelle, F. Cau, and L. Tremblay, "Nuclear magnetic resonance studies of hydrodynamic effects in a critical binary mixture," The Journal of Physical Chemistry, vol. 95, no. 18, pp. 7071-7078, 1991.

[37] A. I. Nakatani, M. D. Poliks, and E. T. Samulski, "Nmr investigation of chain deformation in sheared polymer fluids," Macromolecules, vol. 23, no. 10, pp. 2686-2692, 1990. 
[38] P. T. Callaghan, "Rheo-nmr: nuclear magnetic resonance and the rheology of complex fluids," Reports on Progress in Physics, vol. 62, no. 4, p. 599, 1999.

[39] _ - "Rheo-nmr and velocity imaging," Current Opinion in Colloid \& Interface Science, vol. 11, no. 1, pp. $13-18,2006$.

[40] - "Rheo-nmr: A new window on the rheology of complex fluids," eMagRes, vol. 1, no. 1, March 2012.

[41] B. Medronho, J. Brown, M. G. Miguel, C. Schmidt, U. Olsson, and petrik Galvosas, "Planar lamellae and onions: a spatially resolved rheo-nmr approach to the shear-induced structural transformations in a surfactant model system," Soft Matter, vol. 7, pp. 4938-4947, 2011.

[42] J. E. Maneval, D. Bernin, H. T. Fabich, J. D. Seymour, and S. L. Codd, "Magnetic resonance analysis of capillary formation reaction front dynamics in alginate gels," Magnetic Resonance in Chemistry, vol. 49, no. 10, pp. 627-640, 2011.

[43] N. Malassagne-Bulgarelli and K. M. McGrath, "Emulsion ageing: effect on the dynamics of oil exchange in oil-in-water emulsions," Soft Matter, vol. 9, pp. 48-59, 2013.

[44] D. Grabowski and C. Schmidt, "Simultaneous measurement of shear viscosity and director orientation of a side-chain liquid-crystalline polymer by rheonmr," Macromolecules, vol. 27, no. 9, pp. 2632-2634, 1994.

[45] K.-F. Ratzsch, C. Friedrich, and M. Wilhelm, "Low-field rheo-nmr: A novel combination of nmr relaxometry with high end shear rheology," Journal of Rheology, vol. 61, no. 5, pp. 905-917, 2017.

[46] M. A. Fardin, T. J. Ober, V. Grenard, T. Divoux, S. Manneville, G. H. McKinley, and S. Lerouge, "Interplay between elastic instabilities and shear-banding: three categories of taylor-couette flows and beyond," Soft Matter, vol. 8, no. 39, pp. $10072-10089,2012$.

[47] H. Mohammadigoushki and S. J. Muller, "A flow visualization and superposition rheology study of shear-banding wormlike micelle solutions," Soft Matter, vol. 12, pp. 1051-1061, 2016.

[48] S. Kuczera, C. Perge, M.-A. Fardin, T. I. Brox, M. A. K. Williams, S. Manneville, and P. Galvosas, "Anomalous shear banding revisited with rheo-nmr and rheo-usv," Rheologica Acta, vol. 54, no. 7, pp. 619-636, Jul 2015. 
[49] J.-B. Salmon, A. Colin, S. Manneville, and F. Molino, "Velocity profiles in shearbanding wormlike micelles," Phys. Rev. Lett., vol. 90, p. 228303, Jun 2003.

[50] S. Lerouge, M.-A. Fardin, M. Argentina, G. Grégoire, and O. Cardoso, "Interface dynamics in shear-banding flow of giant micelles," Soft Matter, vol. 4, no. 9, pp. 1808-1819, 2008.

[51] M. R. Lopez-Gonzalez, W. M. Holmes, and P. T. Callaghan, "Rheo-nmr phenomena of wormlike micelles," Soft Matter, vol. 2, pp. 855-869, 2006.

[52] M. M. Britton and P. T. Callaghan, "Nuclear magnetic resonance visualization of anomalous flow in cone-and-plate rheometry," Journal of Rheology, vol. 41, no. 6, pp. 1365-1386, 1997.

[53] J. J. Kobine and T. Mullin, "Nonlinear phenomena in hybrid couette flow composed of planar and circular shear," Physics of Fluids, vol. 13, no. 6, pp. 15831593, 2001.

[54] N. Tillmark and P. H. Alfredsson, “Turbulence in plane couette flow," Applied Scientific Research, vol. 51, no. 1, pp. 237-241, Jun 1993.

[55] D. Krug, B. Lüthi, H. Seybold, M. Holzner, and A. Tsinober, "3d-ptv measurements in a plane couette flow," Experiments in Fluids, vol. 52, no. 5, pp. 1349-1360, May 2012.

[56] AR 2000 Rheometer: Rheometrics Series Operator's Manual, L ed., TA Instruments, 109 Lukens Drive, New Castle, DE 1972, USA, Jan. 2007.

[57] Rheology Advantage Instrument Control: Rheometrics Series Getting Started Guide, E ed., TA InstrumentsWaters LLC, 109 Lukens Drive, New Castle, DE 1972, USA, Jan. 2004.

[58] C. R. López-Barrón, A. K. Gurnon, A. P. R. Eberle, L. Porcar, and N. J. Wagner, "Microstructural evolution of a model, shear-banding micellar solution during shear startup and cessation," Phys. Rev. E, vol. 89, p. 042301, Apr 2014.

[59] K. W. Feindel and P. T. Callaghan, "Anomalous shear banding: multidimensional dynamics under fluctuating slip conditions," Rheologica Acta, vol. 49, no. 10, pp. 1003-1013, Oct 2010.

[60] S. Kuczera, "Rare velocimetry of shear banded flow in cylindrical couette geometry," phdthesis, Victoria University of Wellington, 2015. 
[61] S. Lerouge, M. A. Fardin, M. Argentina, G. Grégoire, and O. Cardoso, "Interface dynamics in shear-banding flow of giant micelles," Soft Matter, vol. 4, no. 9, p. 1808, 2008.

[62] M. A. Fardin, B. Lasne, O. Cardoso, G. Grégoire, M. Argentina, J. P. Decruppe, and S. Lerouge, "Taylor-like vortices in Shear-Banding flow of giant micelles," Phys. Rev. Lett., vol. 103, no. 2, p. 028302, Jul. 2009.

[63] J.-F. Berret, G. Porte, and J.-P. Decruppe, "Inhomogeneous shear flows of wormlike micelles:ma master dynamic phase diagram," Phys. Rev. E, vol. 55, pp. 1668-1676, Feb 1997.

[64] D. Barkley and L. S. Tuckerman, "Turbulent-laminar patterns in plane couette flow," in IUTAM Symposium on Laminar-Turbulent Transition and Finite Amplitude Solutions, T. Mullin and R. Kerswell, Eds. Dordrecht: Springer Netherlands, 2005, pp. 107-127.

[65] R. B. Bird, R. C. Armstrong, and O. Hassager, Dynamics of Polymeric Liquids, 2nd ed. John Wiley and Sons, 1987, vol. 1 Fluid Mechanics. 


\section{Conference Attendance}

1. S. J. Stevenson, T. I. Brox, and P. Galvosas, The $14^{\text {th }}$ International Conference on Magnetic Resonance Microscopy, poster presentation: Planar Couette flow for magnetic resonance microscopy, Halifax NS Canada, Aug 2017

2. S. J. Stevenson, T. I. Brox, and P. Galvosas, The $9^{\text {th }}$ Australian-Korean Rheology Conference, oral presentation: Planar Couette flow for magnetic resonance microscopy, Sydney NSW Australia, Nov 2017

3. S. J. Stevenson, T. I. Brox, and P. Galvosas, The $11^{\text {th }}$ Australian and New Zealand Society for Magnetic Resonance Conference, poster and oral presentation: Planar Couette flow for magnetic resonance microscopy, Kingscliff NSW Australia, Dec 2017 


\section{Appendix A}

\section{Definitions}

Cone and Plate

Constitutive Equations

Control Volume

Couette flow

Deformation

Elastic(ity)

FOF

FOV

Flow
A shear geometry used in rheology (and rheo-NMR) which can achieve a close approximation to homogeneous flow

An equation which characterises a materials mechanical response, usually a function of stress, strain, time, pressure, temperature (equation connects the cause and effect in a body)

An arbitrary volume used for calculation purposes that is either fixed in space or fixed in the fluid

Simple shear flow between two parallel surfaces in relative motion, named after French physicist Maurice Couette

An change in shape and / or volume of an object (strain)

A deformation behaviour that is reversible

(Field Of Flow) The velocity range of flows to be detected by an NMR velocimetry experiment, this should factor a buffer to prevent wrap-around of velocity information that was above the set detected range

(Field Of View) The physical dimensions for the height and width on an MRI

A deformation which is not fully recoverable 
Flow Curve

Geometry

Gradients

Laminar

MRI

NMR

Non-Newtonian Fluid

PCH

Relaxation Time

Rheology

Rheometer

Rheometry

Secondary Flow

Shear
A plot of stress vs. strain for a material, can be acquired using a rheometer

Rheology The sample container which can also apply a deformation or stress to the sample

The name given to magnetic fields used in NMR systems which vary in time and space, used for imaging and motion encoding

Flow without turbulence

(Magnetic Resonance Imaging) An image collected using NMR, like those done in hospitals, detecting nuclei spin density

(Nuclear Magnetic Resonance) A scientific field that uses the precession of nuclei spins in magnetic fields to study properties and behaviours of matter

Fluid that does not obey Navier-Stokes equations

(Planar cylindrical hybrid) The novel shear geometry that is the main focus of this thesis

(Rheology) For a fluid that obeys the Maxwell model of linear viscoelasticity it is the time for the shear stress to reduce by $1 / e$ after flow is stopped

The study of deformation and flow of matter, in particular for complex fluids

An instrument which measures rheological properties of samples

The experimental technique of measuring rheological properties

The components of flow which are in a plane that is orthogonal to the dominant direction of flow in a system

The relative movement of a layer of material with respect to a parallel adjacent layer 
Shear Rate

Spin

Steady Flow

Strain

Strain Rate

Stress

Superconducting

TC

Turbulence

Viscosity

Voxel
The first time derivative of strain, otherwise called a strain rate

An inherent property of nuclei and electrons which have an associated angular momentum

Flow where each point has a velocity which does not vary in time

Deformation of matter defined quantitatively by the ratio of size of the deformation to the original length of the side being deformed

See shear rate

A force per cross-sectional area acting on matter

A material which below a certain temperature resistance drops to zero and a current can be carried indefinitely without loss

(Taylor-Couette) A shear geometry also referred to as a concentric cylinder geometry used in rheology and rheo-NMR

Flow when the velocity components show random variation

A measure of a fluids resistance to deformation by stress of flow, quantitatively defined as the shear stress divided by the shear rate in steady simple shear flow $\eta$

3D imaging pixel volume 


\section{Appendix B}

\section{Derivations}

Material deformations are not only governed by constitutive relations (linking stress and deformation) but additionally require the considerations of conservation concerning both mass and momentum. For completeness the functional relationship between density, temperature and pressure, the equation of state, is required (a typical approximation is to assume an incompressible fluid at constant temperature such that $\rho=$ constant). The results from the conservation rules can be found in most books on mechanics however in not all are they derived (here we follow a very similar layout used in Macosko [7] and Bird et al. [65]).

\section{B.1 Continuity Equation}

Conversation of mass dictates that the changes in density inside a given region of space is balanced by the mass flux crossing the control volume's closed surface (flow of mass into and out of the region).

Mass can be defined by the sum of individual density elements integrated over the control volume $\mathrm{V}$

$$
m=\int_{V} \rho d V
$$

Allowing the system to flow and velocity $\mathrm{v}$ to transport mass across the surface $\mathrm{S}$ of the control volume we can a change mass equal to the flux

$$
\frac{d m}{d t}=\frac{d}{d t} \int_{V} \rho d V=-\int_{S} n \cdot \rho \boldsymbol{v} d S
$$

where the mass flux is just the volume flux $n \cdot v d S$ times the density, and the normal vector if negative so an increase in $\frac{d m}{d t}$ equates to mass entering the control volume. This can be grouped into a single volume integral using the divergence theorem 
and considering that both the volume enclosed by the surface $S$ is the control volume V.

$$
\begin{aligned}
\frac{d}{d t} \int_{V} \rho d V & =-\int_{V}(\nabla \cdot \rho \boldsymbol{v}) d V \\
\int_{V}\left(\frac{d \rho}{d t}+\nabla \cdot \rho \boldsymbol{v}\right) d V & =0
\end{aligned}
$$

The integral must hold for an arbitrary $\mathrm{V}$ so we can choose to shrink the control volume to zero. What is produced as a result is the continuity equation:

$$
\frac{d \rho}{d t}=-\nabla \cdot \rho \boldsymbol{v}
$$

Through the expansion of the divergent term using vector calculus it follows that for an incompressible fluid ( $\rho$ is constant) the equation of continuity becomes

$$
\begin{aligned}
\frac{d \rho}{d t} & =-\rho \nabla \cdot \boldsymbol{v}-\boldsymbol{v} \cdot \nabla \rho \\
\nabla \cdot \boldsymbol{v} & =0
\end{aligned}
$$

Which is equation (2.4) in text. This can be expressed in terms of different coordinate systems as:

$$
\begin{array}{rlrlrl}
\frac{\partial v_{x}}{\partial x}+\frac{\partial v_{y}}{\partial y}+\frac{\partial v_{z}}{\partial z} & =0 & \text { Cartesian } & (x, y, z) \\
\frac{1}{r} \frac{\partial\left(r v_{r}\right)}{\partial r}+\frac{1}{r} \frac{\partial v_{\theta}}{\partial \theta}+\frac{\partial v_{z}}{\partial z}=0 & \text { cylindrical } & (r, \theta, z) \\
\frac{1}{r^{2}} \frac{\partial\left(r^{2} v_{r}\right)}{\partial r}+\frac{1}{r \sin \theta} \frac{\partial\left(v_{\theta} \sin \theta\right)}{\partial \theta}+\frac{1}{r \sin \theta} \frac{\partial v_{\phi}}{\partial \phi}=0 & \text { spherical } & (r, \theta, \phi)
\end{array}
$$

\section{B.2 Momentum Balance Equation}

Conservation of momentum dictates that the change in momentum inside a control volume $\mathrm{V}$ is balanced by the momentum entering through convection and surface contact forces, and the change in momentum due to body forces (i.e. electromagnetic and gravity). Firstly the momentum of a control volume can be described as the continuous sum of infinitesimal volume elements with density $\rho$ and velocity $v$.

$$
m \boldsymbol{v}=\int_{V} \rho \boldsymbol{v} d V
$$

We can therefore inscribe the rate of change of momentum balance equation below. The terms on the right of the equals sign are (from left to right), convection, contact, and body. Body forces are assumed only being due to gravity $g$, contact 
forces can be written as the stress acting on the surface, and convection is the volume flux through the surface $S$ times the momentum per unit volume.

$$
\frac{d}{d t} \int_{V} \rho \boldsymbol{v} d V=-\int_{S}(n \cdot \boldsymbol{v}) \rho \boldsymbol{v} d S+\int_{S} n \cdot \sigma d S+\int_{V} \rho g d V
$$

Applying the divergence theorem all terms can be convert to be in terms of a sum over the control volume.

$$
\frac{d}{d t} \int_{V} \rho \boldsymbol{v} d V=-\int_{V} \nabla \cdot \rho \boldsymbol{v} \boldsymbol{v} d V+\int_{V} \nabla \cdot \sigma d V+\int_{V} \rho g d V
$$

As the control volume is stationary with respect to time the transport theorem can be applied to the left hand side of the equation. Similar with mass conversation we can combine the control, volume integrals.

$$
\int_{V}\left(\frac{d}{d t} \rho \boldsymbol{v}+\nabla \cdot \rho \boldsymbol{v} \boldsymbol{v}-\nabla \cdot \sigma-\rho g\right) d V=0
$$

The control volume is arbitrary hence so can be taken to zero. Rearranging the result gives

$$
\frac{d}{d t} \rho \boldsymbol{v}+\nabla \cdot \rho \boldsymbol{v} \boldsymbol{v}=\nabla \cdot \sigma+\rho g
$$

Equation (2.3) in text is a special incompressible laminar version of this. Application of a vector calculus identity and using the result from mass conservation this can be transformed to

$$
\rho \frac{d}{d t} \boldsymbol{v}+(\rho \boldsymbol{v} \nabla) \cdot \boldsymbol{v}=\nabla \cdot \sigma+\rho g
$$

This can be expressed in terms of different coordinate system as: 


\section{Cartesian}

$x:$

$$
\begin{aligned}
& \rho\left(\frac{\partial v_{x}}{\partial t}+v_{x} \frac{\partial v_{x}}{\partial x}+v_{y} \frac{\partial v_{x}}{\partial y}+v_{z} \frac{\partial v_{x}}{\partial z}\right) \\
& \quad=-\frac{\partial p}{\partial x}+\frac{\partial \tau_{x x}}{\partial x}+\frac{\partial \sigma_{y x}}{\partial y}+\frac{\partial \sigma_{z x}}{\partial z}+\rho g_{x}
\end{aligned}
$$

$y$ :

$$
\begin{aligned}
& \rho\left(\frac{\partial v_{y}}{\partial t}+v_{x} \frac{\partial v_{y}}{\partial x}+v_{y} \frac{\partial v_{y}}{\partial y}+v_{z} \frac{\partial v_{y}}{\partial z}\right) \\
& \quad=-\frac{\partial p}{\partial y}+\frac{\partial \sigma_{x y}}{\partial x}+\frac{\partial \tau_{y y}}{\partial y}+\frac{\partial \sigma_{z y}}{\partial z}+\rho g_{y}
\end{aligned}
$$

$z:$

$$
\begin{aligned}
& \rho\left(\frac{\partial v_{z}}{\partial t}+v_{x} \frac{\partial v_{z}}{\partial x}+v_{y} \frac{\partial v_{z}}{\partial y}+v_{z} \frac{\partial v_{z}}{\partial z}\right) \\
& =-\frac{\partial p}{\partial z}+\frac{\partial \sigma_{x z}}{\partial x}+\frac{\partial \sigma_{y z}}{\partial y}+\frac{\partial \tau_{z z}}{\partial z}+\rho g_{z}
\end{aligned}
$$

\section{Cylindrical}

$$
\begin{aligned}
r & : \\
& \rho\left(\frac{\partial v_{r}}{\partial t}+v_{r} \frac{\partial v_{r}}{\partial r}+\frac{v_{\theta}}{r} \frac{\partial v_{r}}{\partial \theta}+v_{z} \frac{\partial v_{r}}{\partial z}-\frac{v_{\theta}^{2}}{r}\right) \\
& =-\frac{\partial p}{\partial r}+\frac{1}{r} \frac{\partial}{\partial r}\left(r \tau_{r r}\right)+\frac{1}{r} \frac{\partial \sigma_{\theta r}}{\partial \theta}+\frac{\partial \sigma_{z r}}{\partial z}-\frac{\tau_{\theta \theta}}{r}+\rho g_{r} \\
\theta & : \\
\rho( & \left.\frac{\partial v_{\theta}}{\partial t}+v_{r} \frac{\partial v_{\theta}}{\partial r}+\frac{v_{\theta}}{r} \frac{\partial v_{\theta}}{\partial \theta}+v_{z} \frac{\partial v_{\theta}}{\partial z}+\frac{v_{r} v_{\theta}}{r}\right) \\
& =-\frac{1}{r} \frac{\partial p}{\partial \theta}+\frac{1}{r^{2}} \frac{\partial}{\partial r}\left(r^{2} \sigma_{r \theta}\right)+\frac{1}{r} \frac{\partial \tau_{\theta \theta}}{\partial \theta}+\frac{\partial \sigma_{\theta z}}{\partial z}+\frac{\sigma_{\theta r}-\sigma_{r \theta}}{r}+\rho g_{\theta} \\
z & : \\
\rho( & \left.\frac{\partial v_{\phi}}{\partial t}+v_{r} \frac{\partial v_{\phi}}{\partial r}+\frac{v_{\theta}}{r} \frac{\partial v_{\phi}}{\partial \theta}+v_{z} \frac{\partial v_{z}}{\partial z}\right) \\
& =-\frac{\partial p}{\partial z}+\frac{1}{r} \frac{\partial}{\partial r}\left(r \sigma_{r z}\right)+\frac{1}{r} \frac{\partial \sigma_{\theta z}}{\partial \theta}+\frac{\partial \tau_{z z}}{\partial z}+\rho g_{z}
\end{aligned}
$$




\section{Spherical}

$$
\begin{aligned}
r & : \\
& \rho\left(\frac{\partial v_{r}}{\partial t}+v_{r} \frac{\partial v_{r}}{\partial r}+\frac{v_{\theta}}{r} \frac{\partial v_{r}}{\partial \theta}+\frac{v_{\phi}}{r \sin \theta} \frac{\partial v_{r}}{\partial \phi}-\frac{v_{\theta}^{2}+v_{\phi}^{2}}{r}\right) \\
& =-\frac{\partial p}{\partial r}+\frac{1}{r^{2}} \frac{\partial}{\partial r}\left(r^{2} \tau_{r r}\right)+\frac{1}{r \sin \theta} \frac{\partial}{\partial \theta}\left(\sigma_{\theta r} \sin \theta\right)+\frac{1}{r \sin \theta} \frac{\partial \sigma_{r \phi}}{\partial \phi} \\
& -\frac{\tau_{\theta \theta}+\tau_{\phi \phi}}{r}+\rho g_{r}
\end{aligned}
$$

$\theta:$

$$
\begin{aligned}
& \rho\left(\frac{\partial v_{\theta}}{\partial t}+v_{r} \frac{\partial v_{\theta}}{\partial r}+\frac{v_{\theta}}{r} \frac{\partial v_{\theta}}{\partial \theta}+\frac{v_{\theta}}{r \sin \theta} \frac{\partial v_{\theta}}{\partial \phi}+\frac{v_{r} v_{\theta}}{r}-\frac{v_{\phi}^{2} \cot \theta}{r}\right) \\
& =-\frac{1}{r} \frac{\partial p}{\partial \theta}+\frac{1}{r^{3}} \frac{\partial}{\partial r}\left(r^{3} \sigma_{r \theta}\right)+\frac{1}{r \sin \theta} \frac{\partial}{\partial \theta}\left(\tau_{\theta \theta} \sin \theta\right)+\frac{1}{r \sin \theta} \frac{\partial \sigma_{\phi \theta}}{\partial \phi} \\
& \quad+\frac{\sigma_{\theta r}-\sigma_{r \theta}-\cot \theta \tau_{\phi \phi}}{r}+\rho g_{\theta}
\end{aligned}
$$

$\phi:$

$$
\begin{aligned}
& \rho\left(\frac{\partial v_{\phi}}{\partial t}+v_{r} \frac{\partial v_{\phi}}{\partial r}+\frac{v_{\theta}}{r} \frac{\partial v_{\phi}}{\partial \theta}+\frac{v_{\phi}}{r \sin \theta} \frac{\partial v_{\phi}}{\partial \phi}+\frac{v_{\phi} v_{r}}{r}+\frac{v_{\theta} v_{\phi}}{r} \cot \theta\right) \\
& \quad=-\frac{1}{r \sin \theta} \frac{\partial p}{\partial \phi}+\frac{1}{r^{3}} \frac{\partial}{\partial r}\left(r^{3} \sigma_{r \phi}\right)+\frac{1}{r \sin \theta} \frac{\partial}{\partial \theta}\left(\sigma_{\theta \phi} \sin \theta\right)+\frac{1}{r \sin \theta} \frac{\partial \tau_{\phi \phi}}{\partial \phi} \\
& \quad+\frac{\sigma_{\phi r}-\sigma_{r \phi}+2 \cot \theta \sigma_{\theta \phi}}{r}+\rho g_{\phi}
\end{aligned}
$$

